\title{
Modulating the aggregation of alpha-synuclein and prion protein with small molecules.
}

\author{
Dissertation \\ for the award of the degree \\ "Doctor of Philosophy" Ph.D. Division of the Mathematics and Natural Sciences \\ of the Georg-August-Universität Göttingen \\ within the doctoral program of Biology \\ of the Georg-August University School of Science (GAUSS)
}

submitted by

Luis Eduardo Fonseca Ornelas

from Acapulco, Mexico

Göttingen, 2016 


\section{Thesis committee}

Prof. Dr. Markus Zweckstetter, NMR-based Structural Biology, Max Planck Institute for Biophysical Chemistry

Prof. Dr. Tiago Outeiro, Department of Neurodegeneration and Restaurative Research, University Medical Center Göttingen

Prof. Dr. Henning Urlaub, Bioanalytical Mass Spectrometry, Max Planck Institute for Biophysical Chemistry

\section{Members of the examination board}

Prof. Dr. Reinhard Jahn, Department of Neurobiology, Max Planck Institute for Biophysical Chemistry

Prof. Dr. Christian Griesinger, NMR-based Structural Biology, Max Planck Institute for Biophysical Chemistry

Prof. Dr. Kai Tittmann, Department of Molecular Enzymology, Georg August University Göttingen

Date of the oral examination: Monday, March 14 $4^{\text {th }}, 2016$ 
This cumulative thesis is based on the following publications

Fonseca-Ornelas L, Eisbach SE, Paulat M, Giller K, Fernandez CO, Outeiro TF, Becker S, Zweckstetter M. Small molecule-mediated stabilization of vesicleassociated helical a-synuclein inhibits pathogenic misfolding and aggregation. Nat. Commun. 2014. 5:5857 doi: 10.1038/ncomms6857.

Skora L, Fonseca-Ornelas L, Hofele RV, Riedel D, Giller K, Watzlawik J, SchulSchaeffer WJ, Urlaub H, Becker S, Zweckstetter M. Burial of the polymorphic residue 129 in amyloid fibrils of prion stop mutants. J. Biol. Chem. 2013. 288(5): 2994-3002.

Fonseca-Ornelas L, Fernandez CO, Becker S, Zweckstetter M. Small moleculeinduced formation of helical alpha-synuclein oligomers (in preparation). 
I hereby declare that the thesis "Modulating the aggregation of alpha-synuclein and prion protein by small molecules" has been written independently and with no other sources and aids than quoted. 


\section{Acknowledgements}

I would like to show my deep and sincere appreciation for Prof. Dr. Markus Zweckstetter. He provided me with a great opportunity to work in exciting projects and gave me the chance and encouragement to develop my own way of thinking and researching. It has certainly been a once-in-a-lifetime thing, and for that I will be forever grateful.

I would also like to thank Prof. Dr. Christian Griesinger. The facilities and resources that I was able to use during my $\mathrm{PhD}$ are first level. I just hope I did not get too spoiled by them.

Prof. Dr. Tiago Outeiro and Prof. Dr. Henning Urlaub for very fruitful collaborations and for being part of my thesis committee.

Prof. Dr. Christian Griesinger, Prof. Dr. Reinhard Jahn, and Prof. Dr. Kai Tittmann, for agreeing to be part of my examination board.

Dr. Stefan Becker for all the support in the laboratory and the always-encouraging words.

Karin Giller and Maria Paulat for amazing support in the laboratory and for beautifully purified proteins.

Gudrun Heim and Dr. Dietmar Riedel for acquiring electron micrographs.

Dr. Romina Hofele for instrumental mass spectrometry experiments and great friendship.

Dr. Claudio O. Fernández for his contributions during the development of my $\mathrm{PhD}$.

Dr. Carola Reinhard for her unmatched support and effort during my time as a Marie Curie fellow. 
Dr. Martin Schwalbe, Dr. Francesca Munari, Dr. Nasrollah Rezaei-Ghaleh and Dr. Elias Akoury for teaching me the basics of NMR when I knew nothing about it.

Florencia Gillanders, for her idea of using solvatochromic dyes in my project and her support both in and out of the work environment.

The co-workers who became friends: Mario, Aldo, Filippo, Elias, Yunior, Yao, Martin, Francesca, Hari, Timo, Piotr, and many others.

The whole NMR2 department, for creating a nice working environment.

To my parents, for blindly believing in me.

Sofía Battaglia, for pushing me to be a better person. For her love. 


\section{Abstract}

As living and health conditions improve in modern societies, the percentage of older people rises. A byproduct of this ageing is the marked increase of cases of cancer, cardiovascular, and neurodegenerative diseases. Many neurodegenerative diseases share a common hallmark: Protein misfolding and aggregation that either drives or accompanies neuronal death in discrete or widespread brain regions. Finding ways to inhibit protein aggregation is thus a key step in preventing neurodegenerative diseases. Out of these, Parkinson's disease (PD) is the second most common disorder, affecting more than five million people worldwide. Its most common symptoms include slowness of movement, rigidity, resting tremor, and postural instability. At the tissue level, PD is characterized by a profound loss of dopaminergic neurons in the substantia nigra of the brain. This loss generates a lack of dopamine in the striatum and a concomitant deregulation of the limbic system that causes the main symptoms in PD patients. Closer examination of the substantia nigra reveals the formation of protein aggregates in which the main component is a misfolded form of the protein alpha-synuclein. This, together with the fact that triplications and point mutations in the alpha-synuclein gene are associated with familial forms of PD makes alphasynuclein an optimal target for research. As part of this work we have now discovered that the first steps of alpha-synuclein aggregation follow significantly different pathways in a lipid-based environment versus aggregation in solution, and that different small chemical compounds display distinct anti-aggregation properties depending on the presence or absence of lipid vesicles. We further demonstrated that the aggregation of vesicle-bound alpha-synuclein depends on the insertion of the hydrophobic domain into the membrane. The insertion of alpha-synculein into the 
membrane can be prevented by a small chemical compound, opening a novel approach to block aggregation and toxicity of alpha-synuclein.

The second part of my thesis revolves around the aggregation properties of a small peptide from the prion protein $(\mathrm{PrP})$. We have shown that the residue at position 129 plays a central role in its aggregation and that a region spanning from residues 111-137 form a strongly solvent-protected segment, with residues 111 and 135 being in close spatial proximity. We have also demonstrated that the protonation state of a histidine residue in position 111 critically impacts the aggregation of this prion peptide. Furthermore, we showed that sequence homology within the solventprotected region is of utmost importance for the height of the species barrier, a central aspect of PrP biology. 


\section{Table of contents}

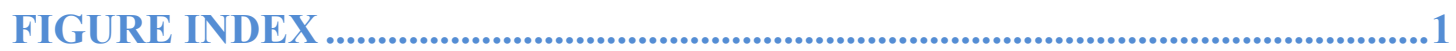

ABBREVIATIONS ................................................................................................2

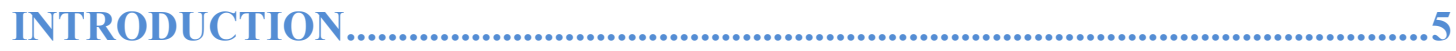

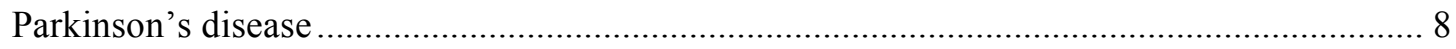

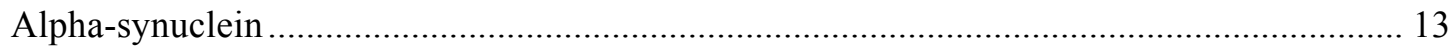

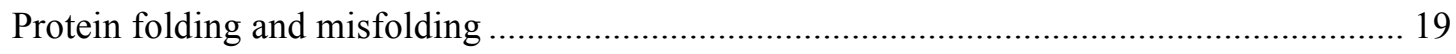

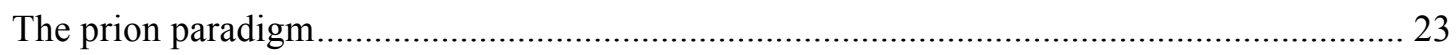

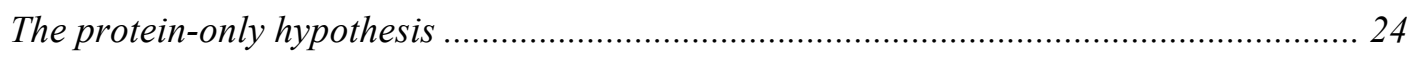

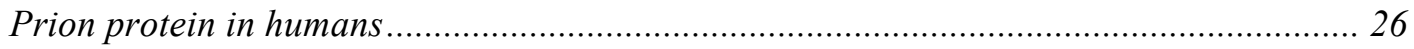

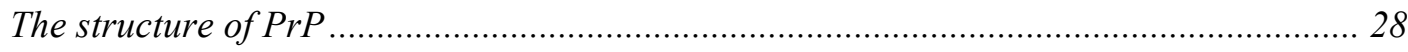

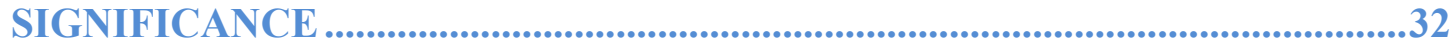

SELECTED METHODS ......................................................................................33

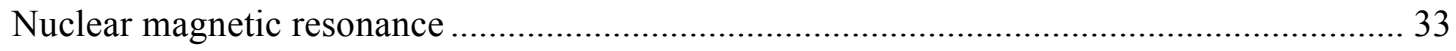

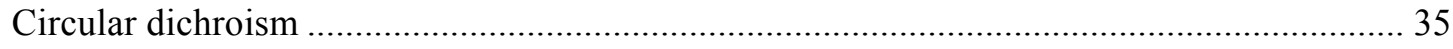

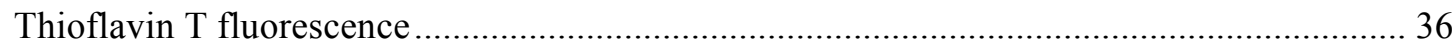

REFERENCES

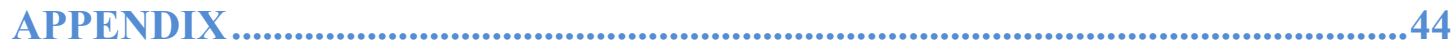

The protonation state of Histidine 111 critically influences the aggregation of the evolutionary conserved, central region of the human prion protein ..................................... 44

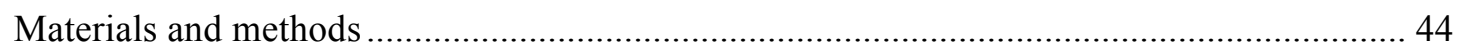

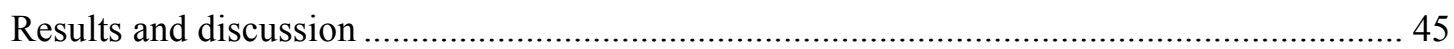


Histidine 111 and 140 have similar pKa values in the monomeric, unfolded state of humPrP(108-143)

Importance of histidine 111 and 140 for amyloid formation of humPrP(108-143).....

Influence of sequence homology at position 138/139 for seeding of prion aggregation .... 51

Conclusions. 53

Appendix references 53

Small molecule mediated stabilization of vesicle-associated helical $\alpha$-synuclein inhibits pathogenic misfolding and aggregation

Burial of the polymorphic residue 129 in amyloid fibrils of prion stop mutants.92

Curriculum vitae 


\section{FIGURE INDEX}

Figure 1. Percent of Population Aged 60 AND OVER, 65 AND OVER, AND 80 AND OVER 6

FIGURE 2. SCHEMATIC REPRESENTATION OF FIBRIL FORMATION AND ITS PHASES ..........8

Figure 3. MESENPHALIC DOPAMINE PATHWAYS IN THE HUMAN BRAIN .........................11

FigURE 4. AgGREgATION STAGES OF ALPHA-SYNUCLEIN .......................................... 13

FigURE 5. AMINO ACID AND SCHEMATIC SEQUENCE OF ALPHA-SYNUCLEIN..................15

FigURE 6. 3D-STRUCTURE OF ALPHA-SYNUCLEIN BOUND TO SDS MICELLES................17

FIGURE 7. ENERGY LANDSCAPE FOR PROTEIN FOLDING...............................................22

FigURE 8. EARLY DRAFT OF THE CENTRAL DOGMA OF MOLECULAR BIOLOGY...............24

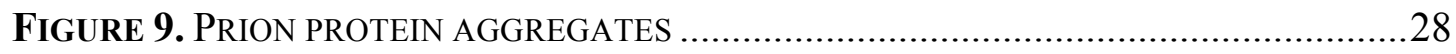

FigURE 10. SCHEMATIC REPRESENTATION OF THE MAIN REGIONS IN PRION PROTEIN ...30

FIGURE 11. CARTOON OF THE THREE-DIMENSIONAL STRUCTURE OF THE HUMAN PRION

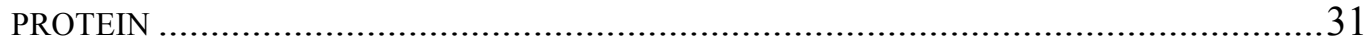

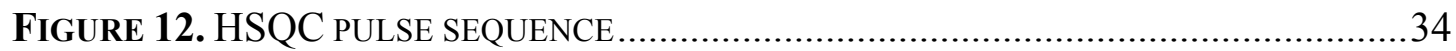

FIGURE 13. MAIN REGIONS AND CHARACTERISTICS OF THE PRION PROTEIN..................46

FigURE 14. H111 AND H140 ARE EXPOSED TO THE SAME MICROENVIRONMENT ..........47

FigURE 15. THE PROTONATION STATE OF H111 REGULATES THE AGGREGATION OF HUMPRP(108-143). 49

Figure 16. PePtIDE SEQUENCES USED FOR AGGREGATION SEEDING EXPERIMENTS .....52 


\section{ABBREVIATIONS}

AD

ALS

$\alpha$ Syn

CD

CJD

CSP

CSPalpha

Da

DBS

DMSO

DNA

DOPC

DOPE

DOPS

EDTA

EGCG

FFI

GPI

GSS

GTP

HEPES

HSQC

IDP
Alzheimer's disease

Amyotrophic lateral sclerosis

Alpha-synuclein

Circular dichroism

Creutzfeldt-Jakob disease

Chemical shift perturbation

Cysteine string protein alpha

Dalton

Deep brain stimulation

Dimethyl sulfoxide

Deoxyribonucleic acid

1,2-dioleoyl-sn-glycero-3-phosphocholine

1,2-dioleoyl-sn-glycero-3-phosphoethanolamine

1,2-dioleoyl-sn-glycero-3-phospho-L-serine

Ethylenediaminetetraacetic acid

Epigallocatechin gallate

Familial fatal insomnia

Glycophosphatidylinositol

Gerstmann-Sträussler-Scheinker syndrome

Guanosine-5'-triphosphate

4-(2-hydroxyethyl)-1-piperazineethanesulfonic acid

Heteronuclear single quantum coherence

Intrinsically disordered protein 
INEPT

L-DOPA

LB

LDH

LUV

ml

mM

$\mu \mathrm{M}$

MS

NAC

nm

nM

NMR

PcTS

PD

POPA

POPC

PrP

PrPc

PrPres

PrPsc

RF

RNA

SDS

SN
Insensitive nuclei enhanced by polarization transfer

L-3,4-dihydroxyphenylalanine

Lewy body

Lactate dehydrogenase

Large unilamellar vesicle

Milliliter

Millimolar

Micromolar

Mass spectrometry

Non-amyloid component

Namometer

Nanomolar

Nuclear magnetic resonance

Phtahlocyanine tetrasulfonate

Parkinson's disease

1-palmitoyl-2-oleoyl-sn-glycero-3-phosphate

1-palmitoyl-2-oleoyl-sn-glycero-3-phosphocholine

Prion protein

Cellular prion protein

Resistant prion protein

Scrapie prion protein

Radio frequency

Ribonucleic acid

Sodium dodecyl sulfate

Substantia nigra 
SNARE

SUV

$\tau$

TEM

ThT

TOCSY

Tris

TROSY

TSE

UV

vCJD

VPSPr
SNAP (soluble NSF attachment protein) receptor

Small unilamelar vesicle

Tau

Transmission electron microscopy

Thioflavin T

Total correlation spectroscopy

Hydroxymethyl aminomethane

Transverse relaxation optimized spectroscopy

Transmissible Spongiform Encephalopathies

Ultraviolet

Variant Creutzfeldt-Jakob disease

Variable protease-sensitive prionopathy 


\section{INTRODUCTION}

The increase in life expectancy that human population saw over the course of the $20^{\text {th }}$ century has been, without doubt, one of the biggest accomplishments of our kind. Although trends differ between countries, a clear upwards tendency has steadily prevailed among them, to a point in which babies born after the year 2000 will likely live well into their eighties and more ${ }^{1,2}$. The reasons for such a stark increase in life expectancy have different sources -such as a tremendous decrease in infant mortality rates- and the development of antibiotics that can easily treat and cure infections that would have otherwise killed many persons (as it used to happen not so long ago). The clear result of this is that the population is much more aged now than it was a century ago and, with the tendency showing no sings of imminent decline, this points to a population that, within the next century, will be very rich in sexa, septua and octogenarians (Figure 1) ${ }^{1,2}$. Unfortunately, for cardiovascular diseases and many types of cancer, age clearly represents the greatest risk factor. Neurodegenerative diseases such as Alzheimer and Parkinson's also display a logarithmic increase on its incidence once the age hits the 60 years mark ${ }^{3}$. So, while the danger of dying during childhood or young to mid age due to common infections, for example, has strongly subdued, many diseases characteristic of old-aged people will become more and more prominently a topic of public health because of an increasing number of older adults consuming a significant part of health care resources ${ }^{2,4,5}$. 


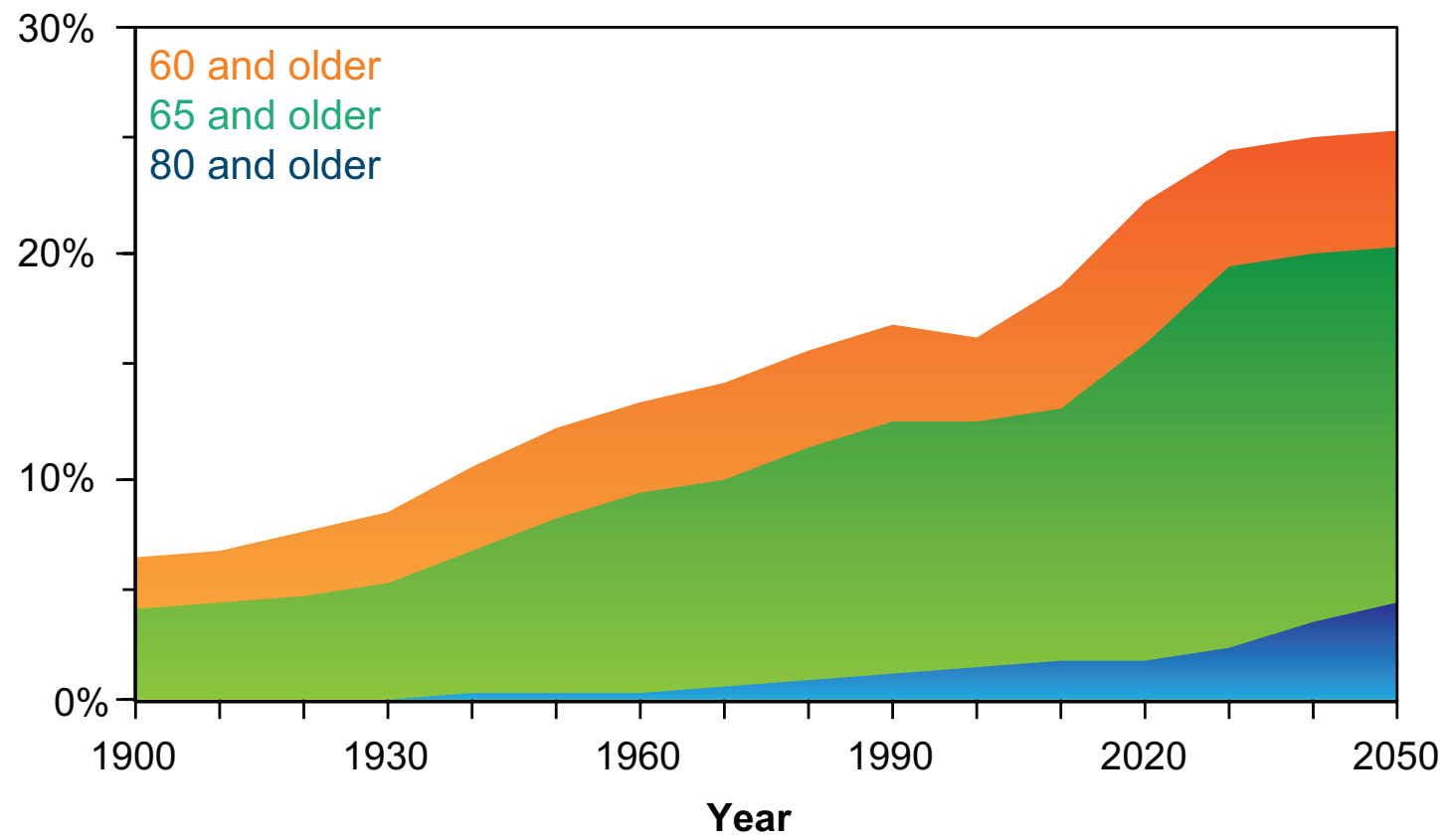

Figure 1. Percent of population aged 60 and over, 65 and over, and 80 and over. Past census and future estimates (based on reference 1).

Of particular interest for this thesis are neurodegenerative diseases. Neurodegenerative diseases related to age include, among others, Alzheimer's disease (AD), Parkinson's disease (PD), amyotrophic lateral sclerosis (ALS), and CreutzfeldtJakob disease (CJD). Despite the fact that all those diseases vary greatly in their prevalence among the population, the symptoms that the affected patients present, as well as in their pathological characteristics and the type of neurons they affect, there seems to be one very important pathogenic mechanism shared among them all: the misfolding and aggregation into histopathologically appreciable amyloid lesions of normally soluble, disease-specific proteins. Beta-amyloid and tau in the case of AD, alpha-synuclein in $\mathrm{PD}$, and the prion protein in $\mathrm{CJD}^{6,7}(\mathrm{~A}$ detailed table containing a list of human diseases associated with misfolded amyloids is in the appendix 1). Interestingly, the anatomical distribution of these deposits of aggregated misfolded proteins often overlaps with areas where neurodegeneration is most extreme ${ }^{8}$. 
The widely accepted model for the aggregation of proteins implies a sigmoidal kinetics process (Figure 2) where the first phase corresponds to a nucleation growth in which a monomer misfolds (thus losing the "native" folding and function) and associates with others to form a nucleus. After the misfolded species increases in number and reaches a critical concentration, it promotes the development of protofibrils, a transient state between the oligomeric and fibrillar forms. The primary growth process is often attributed to fibril-end elongation by a dock-lock mechanism by which misfolded oligomeric species polymerize the growth of the fibril by unitaddition, changing from oligomeric to fibrilar species in the process. Fragmentation of mature fibrils can also occur and these small fragments can then serve as seeds, scaffolds upon which new fibrils can grow by feeding on small, preformed misfolded oligomers. These processes together, by their amplifying, chain-reaction nature, are the ones that confer the aggregation a logarithmic growth during the elongation phase $^{9}$. Though still a matter of debate, increasing evidence suggests that the final, stable, fibrillar form acquired during the aggregation course is in fact not primary responsible for the damage done to the cells. Indeed, the latest indications seem to point towards an oligomeric, intermediate state that presents a major challenge for the cell to cope with (such as toxicity through gain-of-function or toxicity through lossof-function) and, as a result, the cell dies ${ }^{2,10-15}$. 


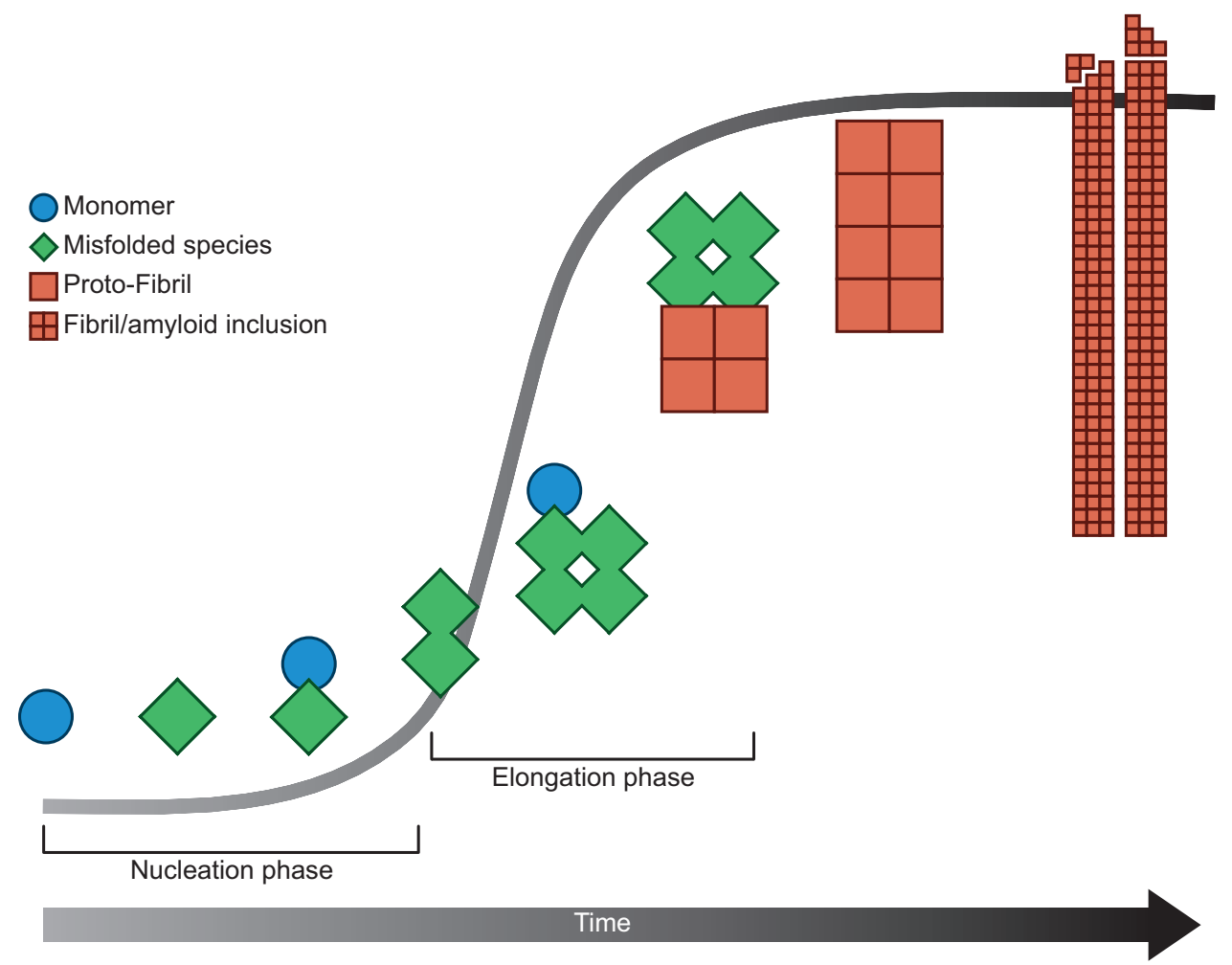

Figure 2. Schematic representation of fibril formation and its phases. Based on references 1 and 9.

\section{Parkinson's disease}

The English physician James Parkinson described the symptoms of what we now call Parkinson's disease for the first time in 1817 to define what he called a shaking palsy $^{3,16}$. Jean-Martin Charcot would later name the ailment after him as Parkinson's disease in 1872. Important contributions in the pathological characterization of the disease would come from Blocq and Marinesco, and Friedrich Lewy, who identified what would later be the hallmark of the disease: the Lewy bodies (LB) $)^{2,4,5,17,18}$. Taken together, their studies allowed determining that the main culprit for PD symptoms arises from the loss of neurons within the substantia nigra of the brain and that the most prevalent characteristic on the patients' brain is the accumulation of eosinophilic aggregates surrounding the affected area, the Lewy bodies. Also vitally important in 
the history of PD were the contributions made by Ehringer and Hornykiewicz, who showed that dopamine is indeed a neurotransmitter in the brain (and not only an intermediate in the synthesis of adrenaline and noradrenaline) and that it plays an important role in the pathogenesis of $\mathrm{PD}^{1,19,20}$

PD is the second most common neurodegenerative disorder. It emerges mainly in people older than 60 , with a prevalence close to $1 \%$ within this age range. Such prevalence rises steadily until it reaches $5 \%$ in people of 85 and older ${ }^{6,7,21,22}$. With a mean duration of 15 years from time of diagnosis until death, PD is considered a slowly progressive disorder, with some affected individuals surviving two decades or longer. It is also normally regarded that the onset of the disease begins 10 to 15 years before any symptoms are readily noticeable (and well before any diagnosis can be accurately made). Even though the last stages of a PD patient's life tend to be exceedingly complicated because of diverse factors such as development of resistance to the medications as well as the progress of advanced states of dementia, it is also worth noting that PD itself is not fatal. Some of the deaths can certainly arise from complications generated indirectly by $\mathrm{PD}$, such as the difficulty swallowing that can cause food to be aspirated into the lungs -leading to pneumonia and other pulmonary conditions, and movement difficulties that can make people more susceptible to suffer fatal falls $\mathrm{s}^{8,22}$. These are, however, considered a small percentage of the fatalities suffered by persons with PD.

At the clinical level, most patients present symptoms related to movement disorders, including hipokinesia, bradykinesia, resting tremor, rigidity, and postural instability. Cognitive, autonomic, and psychiatric disturbances are also frequent in a 
number of patients, and, though less prevalent, seborrhea (the inability to swallow) and sialorrhea (excessive fat production in the scalp) can also appear. Depression and anxiety that eventually lead to dementia are also present in a significant number of patients in advanced stages of the disease. At the tissue level, the majority of these symptoms can be explained by a profound and selective loss of dopaminergic neurons in the substantia nigra (SN) pars compacta of the brain, a loss so profound indeed that the symptoms appear until close to $80 \%$ of the neurons in this brain structure have already died, or some 15 years after the disease actually begins ${ }^{9,23}$. Since the motor symptoms are generated by the lack of dopaminergic neurons, its effect can be somewhat modulated by dopamine replacement therapy, like L-3,4dihydroxyphenylalanine (L-DOPA) and by neurosurgical procedures such as deep brain stimulation (DBS) that send the electrical inputs that are otherwise absent ${ }^{24}$. Though several treatments show effectiveness in mitigating some of the symptoms caused by PD, currently there is no know cure for this disorder.

The substantia nigra is the most affected region of the brain during the development of PD. The subtantia nigra is a structure located in the mesencephalon, part of the midbrain, which plays a central role in processes involving learning, reward, addiction, and movement. It is divided into two main regions; the pars reticulate (mainly populated by GABAergic neurons) and the pars compacta (occupied mostly by dopaminergic neurons). Forming part of the basal ganglia, the substantia nigra controls movement through a negative loop regulation of the striatum (Figure 3), and it owes its name to a distinctive dark pigmentation coming from the neuromelanin present in the dopaminergic neurons that reside there. Like dopamine (the main produce of the dopaminergic neurons), the precursor of neuromelanin is LDOPA and it is potentially involved in the mitigation of oxidative stress ${ }^{25}$. Due to a 
constellation of difficult symptoms to assay, PD diagnosis is not easily achieved and, as a matter of fact, postmortem observation of depigmentation in the substantia nigra region (due to the death of the dopaminergic neurons within it), together with the positive staining of Lewy bodies, remains, until present day, the only unequivocal diagnosis that a person suffered indeed from $\mathrm{PD}^{26,27}$.

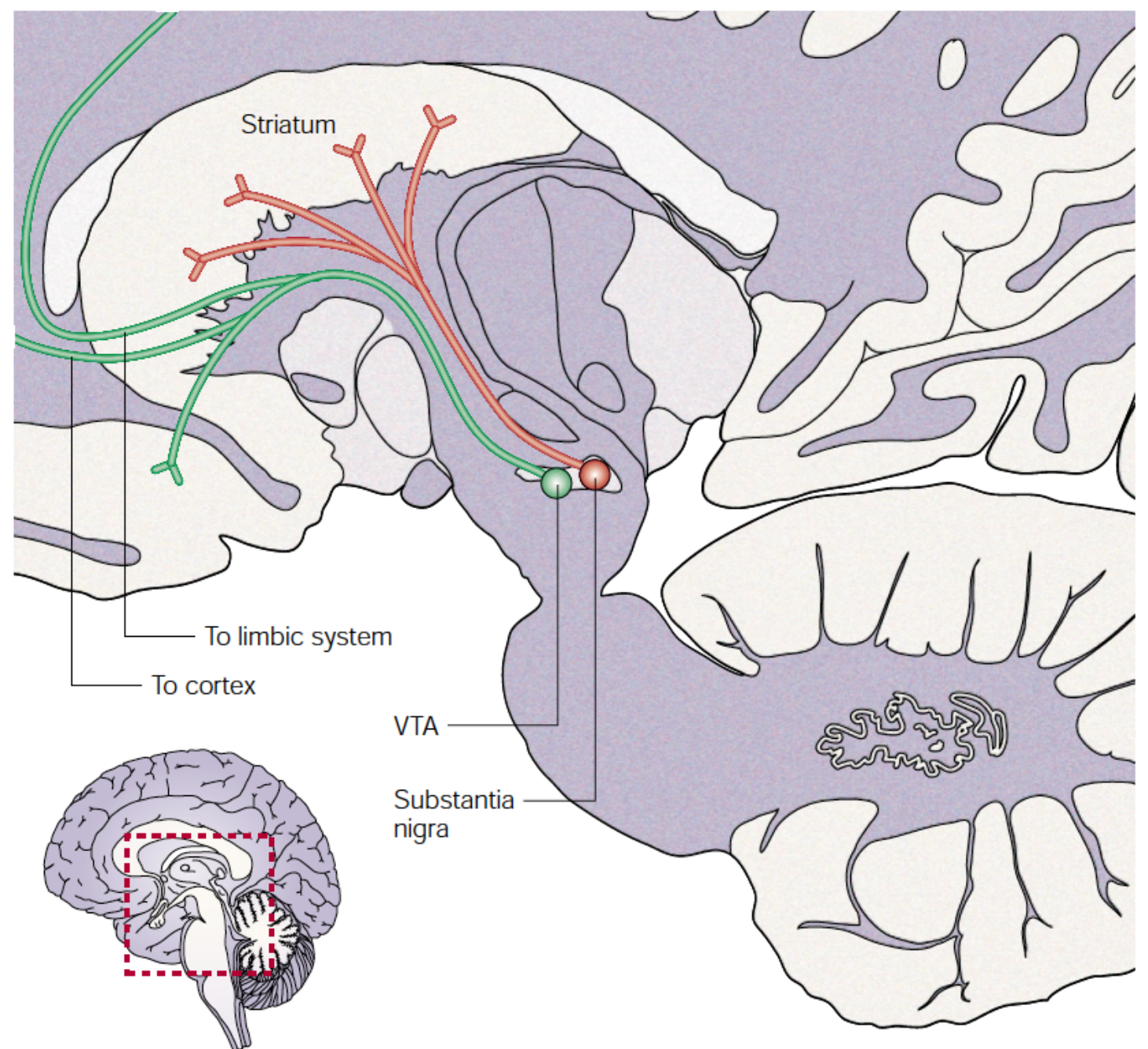

Figure 3. Mesenphalic dopamine pathways in the human brain. The scheme indicates the localization of dopaminergic neurons within the ventral tegmental area (green) and the substantia nigra (red). In PD patients, neurons in the substantia nigra degenerate, giving rise to many of the motor abnormalities characteristic of this disease. From reference 28 . 
The pathological hallmark of PD is the degeneration of dopaminergic neurons in the substantia nigra and the presence of eosinophilic inclusions nowadays called Lewy bodies $^{29}$. The history of Lewy bodies begins in 1912, when Friederich Lewy made use of light microscopy to describe the cellular inclusions characteristic of PD patients. These inclusions, as he pointed out, were not only present in the bodies of neurons but also in the nerve cell processes. Konstantin Nikolaevich Tretiakoff would call them Lewy bodies and Lewy neurites, after him, in $1919^{18}$. Though present in many diseases, the Lewy pathology is still largely known as the hallmark pathological feature of $\mathrm{PD}^{18}$. The role that Lewy bodies play in the pathogenesis of PD has long been a hot topic of discussion among neuropathologists. Whether the Lewy bodies represent the driving insult to the cells, a cytoprotective mechanism, or just an innocuous byproduct of the pathogenesis of PD is still very much a matter of debate ${ }^{30}$.

Biochemical inspection of Lewy bodies reveals a plethora of components. High concentrations of misfolded proteins, uncommon quantities of metals such as copper, iron and zinc, and potentially hazardous amounts of free radicals are commonly present in these aggregates ${ }^{31}$. The accumulation of these harmful particles in a "contained" structure within the cell has led people to believe that the aggregates are either cytoprotective or, at the very least, a way in which the cell tries to deal with elements that otherwise would be toxic ${ }^{30}$. Though the ingredients are numerous and varied and there is not a single recipe for making a Lewy body, it is well accepted that the most pervasive component of it are unbranched filaments of a misfolded/aggregated form of the protein alpha-synuclein ${ }^{32}$ (Figure 4). 
A

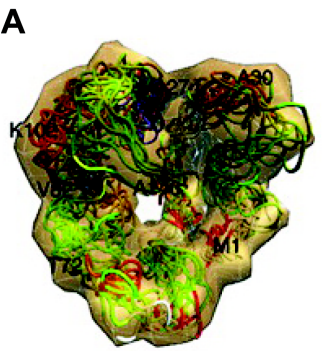

B

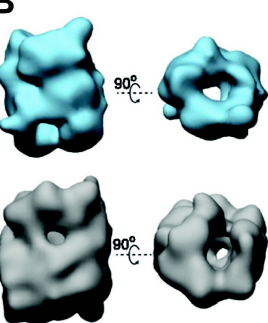

C

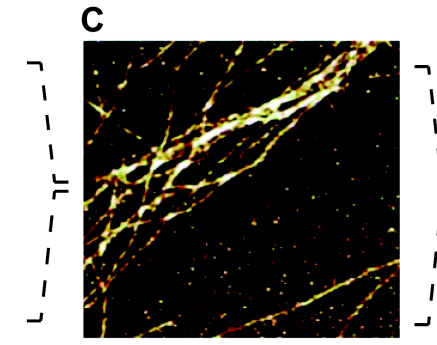

D

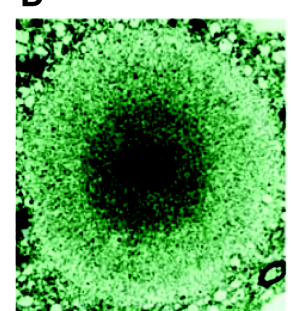

Figure 4. Aggregation stages of alpha-synuclein. From monomers (A) to oligomers

(B) then to fibrils (C) and then to Lewy bodies (D). Taken from references 32 to 35, respectively.

\section{Alpha-synuclein}

Alpha-synuclein was a fairly unknown protein well into the 1990s. Synuclein was first described as a central nervous system protein in the electric ray Torpedo that had a potential homologue in rat. Since it was found localized primarily in synaptic vesicles and in portions of the nucleus, it was named synuclein ${ }^{36}$. It was then found that the non-amyloid-beta component of plaques found in the brains of patients with Alzheimer's disease were constituted primarily by an abundant protein of 140 amino acids that presented a high homology to the torpedo synuclein. Another highly similar protein with 134 amino acids was then found in the human proteome and, due to its homology, they were named alpha and beta-synuclein, respectively ${ }^{37}$. It would later be determined that, in humans, there is yet another synuclein with a slightly different amino acid composition, the gamma-synuclein ${ }^{38,39}$. Afterwards, further indications came to place that an alpha-synuclein homologue present in zebra finch was vital in song learning and, potentially, brain plasticity ${ }^{40}$. It was then described that alphasynuclein was a natively unfolded protein that was capable of acquiring a mostly helical secondary structure upon interaction with lipids ${ }^{41}$. Still not particularly 
famous, two seminal discoveries propelled alpha-synuclein into the international research focus, both of them in 1997: The first was done in the Mihael Polymeropoulos' laboratory, where the group identified an alanine for threonine mutation at position 53 (A53T) within the alpha-synuclein protein that predisposed the members of a family carrying it into developing an autosomal dominant PD phenotype $^{42}$. The second discovery, made soon after that, was done by Maria Spillantini and her collaborators, where they described what was stated in the previous section: that alpha-synuclein was the major component of Lewy bodies in the brains of PD patients ${ }^{43}$. These two discoveries brought together not only the genetic component of $\mathrm{PD}$, but also the misfolding of proteins as a driving mechanism of the disease, both exquisitely converging in the same protein and bringing alphasynuclein into the limelight. The discovery of further mutations that trigger the disease (A30P, E46K, H50Q, G51D, and the previously described A53T) as well as alterations on its promoter that increase the protein levels of alpha-synuclein by threefold also promotes the manifestation of a potent form of PD, only strengthened the notion that alpha-synuclein plays indeed a central role in the acquisition and development of $\mathrm{PD}^{44-48}$.

Alpha-synuclein is a protein present only in vertebrates, consisting of 140 amino acids, lacking both cysteine and tryptophan residues ${ }^{49}$. It is codified by a single gene composed of seven exons localized in the chromosome 4 of the human genome $^{50}$. It is ubiquitously expressed in the body but is particularly enriched in the presynaptic terminals of neurons ${ }^{36,51}$. At the sequence level, it is divided into three main regions: the N-terminal domain, encompassing residues 1-60 and with amphipathic characteristics, the NAC fragment, covering residues 61-95, highly 
amyloidogenic and bearing within it hydrophobic amino-acids, and the C-terminal domain, from residues 96-140, constituting an acidic and mostly negatively charged region $^{49}$. Alpha-synuclein's primary sequence has seven highly conserved imperfect amino acid repeats composed of the consensus sequence KTKEGV (Figure 5). This sequence bares signatures typical of membrane-interacting regions ${ }^{52}$. The most abundant alpha-synuclein species found in Lewy bodies is hyperphosphorylated at S129 (Figure 5) $)^{42,44,45,54}$.

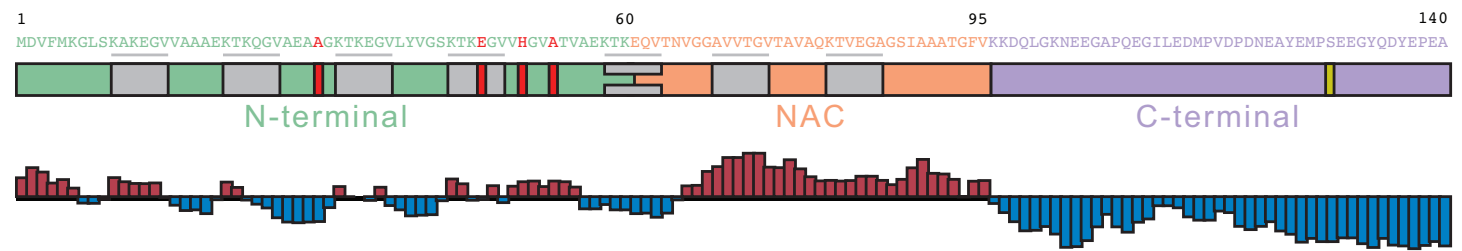

Figure 5. Amino acid and schematic sequence of alpha-synuclein. The three domains are presented in green, orange and purple. KTKEGV repeats are in gray. Positions of mutations that lead to PD are in red. Phosphorylation of S129 is in yellow. The hydropathy index is presented below: blue hydrophilic, red hydrophobic (based on references 41, 54 and others).

In solution, alpha-synuclein is an intrinsically disordered protein (IDP) with no apparent secondary structure ${ }^{41,55}$, but significantly more compact than a simple random coil ${ }^{12}$. Accordingly, it has been proposed that despite this apparent lack of stable secondary structure, important long-range contacts appear between the Cterminal region and the NAC-domain. This contacts imply a non-fully random arrangement in which there is indeed a certain predisposition to form a compact-yet unfolded-ensemble ${ }^{33}$. It has been also demonstrated that alpha-synuclein readily interacts with acidic phospholipids and that, in doing so, it acquires a stable, alpha- 
helical secondary structure ${ }^{56,57}$ (Figure 6). This is characterized by either two alphahelices spanning residues 3-37 and 45-92, or a single, elongated helix spanning until residue $94^{58}$. Regardless of the helix organization, it seems to be clear that the conserved motif KTKEGV plays a central role in the way alpha-synuclein interacts with membranes: the polar residues within the N-terminal region of alpha-synuclein $(\mathrm{S}, \mathrm{E}$, and $\mathrm{K}$ ) face the hydrophilic environment of the cell media, while the hydrophobic residues insert into the membrane. Positively charged residues (K), constitute the boundary between the polar and hydrophobic domains and interact directly with the anionic surface of the phospholipid bilayer ${ }^{51}$. Only the first 94 residues take part in the structural change and, by the same token, only those residues interact with membranes. The C-terminal region, which is negatively charged under physiological conditions, remains highly dynamic and it has been proposed that this region serves as a scaffold upon which other proteins interact and are driven towards the membrane alpha-synuclein is bound to ${ }^{59}$.

Recently, controversial studies mainly proposed by Dennis Selkoe and his collaborators suggest that although alpha-synuclein can certainly be found as an unfolded monomer in vivo, dimeric and, especially tetrameric arrangements are the most prominent species found in living cells. This higher order organization also seems to promote the acquisition of an alpha-helical secondary structure (without the need of a lipid scaffold) and a stark reduction in the aggregation propensity ${ }^{60,61}$. One of their most important conclusions is that finding a way to stabilize alpha-synuclein's acquired secondary structure (trough small chemical compounds, for example) would prevent its aggregation and toxicity. This correlation is still certainly a matter of debate. 


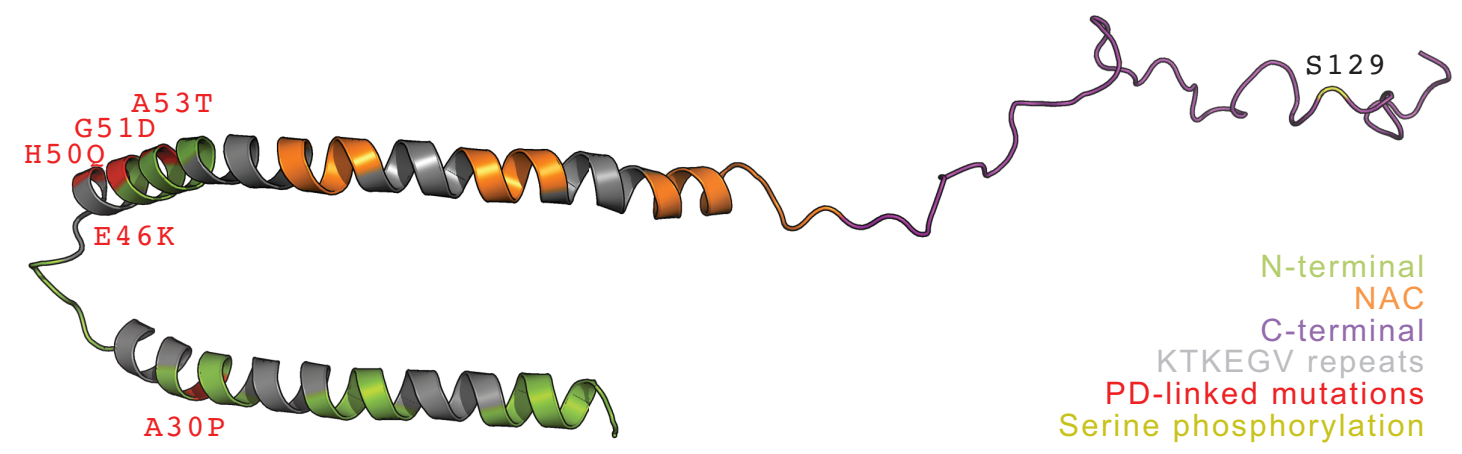

Figure 6. 3D-structure of alpha-synuclein bound to SDS micelles. The same colorcoding from figure 5 is presented. Modified from reference 57.

Alpha-synuclein has been described in a wide array of conditions, and many of its most important properties have been thoroughly investigated. A plethora of reviews regarding alpha-synuclein can be found, and important information is available that covers from structural information all the way to its role in synaptic plasticity and cognition ${ }^{49,51,62}$. The function of alpha-synuclein is, however, controversial to this day. In contrast to many proteins involved in neurodegeneration, which are distributed uniformly throughout the neuron, alpha-synuclein localizes specifically to nerve terminals, being relatively absent in the cell body, dendrites or extra-synaptic sites along the axon ${ }^{40,63}$. In addition, alpha-synuclein is widely expressed in many different types of neurons, suggesting a general role in neuronal function. However, alpha-synuclein is one of the last proteins that localizes to developing synapses, arriving after integral membrane proteins of the synaptic vesicle and the peripheral membrane synapsis proteins ${ }^{64}$. Taken together with the fact that the synuclein family is only present in vertebrates, it becomes apparent that alphasynuclein's presence in the synaptic terminal does not seem to be of essential importance for synaptic development nor for its function ${ }^{52}$. Alpha-synuclein's 
localization to the membrane is also a complicated issue itself, as it lacks a distinct transmembrane domain or a classical lipid anchor that drives it there. A possibility is that alpha-synuclein relies on its $\mathrm{N}$-terminal sequence to promote its binding to membranes. This interaction, however, has proven to be weak, as even in labile extraction methods alpha-synuclein appears mostly as a soluble, unfolded protein, suggesting that although the interaction between alpha-synuclein and membranes does indeed happen, the protein is highly mobile and can potentially function not only when found in contact with membranes but also when it is free in the solution ${ }^{65}$. While alpha-synuclein might not show a strong interaction with lipidic membranes, it is clear that it shows a marked preference for synaptic vesicles. This preference is explained by the high membrane curvature favored by alpha-synuclein, and synaptic vesicles are certainly among the smallest biological membranes described ${ }^{65}$. Interestingly, it has been proposed that alpha-synuclein not only interacts with small vesicles, but that it does so specifically through interaction with lipid rafts ${ }^{65}$, supporting the relevance of this interaction for neurons. A role regarding the uptake of cardiolipin and acyl chain composition has also been suggested, as well as fatty acid uptake and metabolism ${ }^{66}$.

The localization and membrane interactions of alpha-synuclein have long hinted to the possibility of a role in neurotransmitter release and/or synapsis architecture. Indeed, alpha-synuclein knockout mice models show a faster dopamine recovery after repetitive stimulation than wild-type animals, and a consequence of this are reduced dopamine levels in the striatum ${ }^{67}$. Another interesting characteristic is that in alpha-, beta-, and gamma-synuclein triple knockout mice, the presynaptic boutons are significantly smaller ${ }^{68}$. Thus, a role in nerve terminal maintenance, rather than 
neurotransmitter release, may be important ${ }^{69}$. Alpha-synuclein's role as a chaperone has also been proposed, noting that its knockout mouse displays a very similar phenotype as the one presented by cysteine string protein alpha (CSPalpha) knockouts, were the levels of SNARE SNAP-25 are markedly affected ${ }^{70}$. Much of the work done in Thomas Südhof's laboratory seem to point to the possibility of alphasynuclein regulating the general formation of SNARE complexes, not in an absolutely fate-determining fashion, but fine-tuning it ${ }^{69}$.

Another, non-mutually exclusive putative function for alpha-synuclein could arise from the interactions with a plethora of other proteins it interacts with. It has been reported that a multimeric form of alpha-synuclein is able to interact with tubulin and, in doing so, it regulates the microtubule polymerization and, to a greater extent, the local shape of the cytoeskeleton ${ }^{71}$. Another interesting interaction happens with the small GTPases rab3a and rab8a, and it has been proposed that this interaction regulates the ability of alpha-synuclein to recognize and bind to membranes in a GTPdependent mechanism ${ }^{72,73}$. Yet another partner of alpha-synuclein is synphilin-1, which was first described also populating nerve terminals and found also in the Lewy bodies together with alpha-synuclein ${ }^{74}$. The two of them seem to abolish each other's toxicity by promoting the formation of non-reactive aggresomes and diverting the aggregation kinetics towards a less harmful (though apparently still amyloid) state ${ }^{75-}$ 77

\section{Protein folding and misfolding}

The function of a protein is determined by its structure. By the same token, the structure of a protein is, to a great extent, mandated by its amino acid sequence. Thus, 
though a variety of factors are involved directly in the folding of a protein, it is the amino acid sequence the one who carries all the necessary information for protein to acquire its native folding and, by extension, its function. The total number of possible conformations that a given sequence can give rise to is astronomically large and it is now known that a stochastic search in which many intermediate conformational stages are reached first, is a primordial step which reduces complexity of further conformational stages ${ }^{78}$. Given also that, in general, the functional state of a protein involves native interactions between the amino acid residues that conform it and these are the lowest-energy ones, the "correctly" folded polypeptide chain is reached through a folding process within this smaller pool of intermediate states. This "lowest level energy" concept is the one that gives rise to the so-called energy landscape for protein folding (Figure 7), in which any given protein sequence has a limited amount of states in which the energy level is low. This, coupled with natural selection, has allowed the evolution to favor amino acid sequences that code for proteins that fold rapidly and efficiently ${ }^{78}$.

The energy landscape, however, allows under certain circumstances the misfolding of a protein, the achievement of a structure that is not the native, functional one. As stated before, many human diseases arise precisely as a result of this, when a protein falls into an energy level caveat that is not the functional one. Either because in doing so it acquires a novel, toxic function or because when misfolded it loses the ability to perform its normal tasks. Regardless of the originating protein, the fibrillar structures found in many of these diseases share similar morphologies, such as the long, unbranched structures of a few nanometer in diameter, and a classical beta-sheet secondary structure ${ }^{78}$. 
Aggregates and Lewy bodies found in the brains of PD patients show unbranched filaments of aggregated alpha-synuclein with predominant beta-sheet secondary structure. The structural characterization of oligomeric stages has proven to be a more difficult task, mainly because of the high heterogeneity of the samples (the fibrillization process yields diverse components before the fibrillar state is reached) and the often-transient nature of the oligomeric species. Using cryo-electron microscopy, it has recently been shown that, regardless of the large heterogeneity in sizes, alpha-synuclein oligomers appear to have a shared hollow cylindrical architecture $^{34}$ that would also support the previously proposed hypothesis that at least a subset of oligomers can go into membranes and promote the formation of pores that disrupt cellular activities ${ }^{15}$. Since the instability of the oligomers is at the experimental level a difficult issue, using small chemical compounds to slow its transitory nature has also been attempted ${ }^{79}$. Regardless of the employed technique or the method for preparing alpha-synuclein oligomers, there seems to be a common thread in their formation that includes the achievement of beta-sheet strands interconnecting through hydrogen bonds which, while stable, are still different from the ultra-stable cross-beta, steric zipper arrangements found in mature fibrils ${ }^{34,80,81}$. Very recent X-ray crystallography data of a small alpha-synuclein peptide has shed atomic-level resolution of how these proto-fibrils $\operatorname{look}^{82}$, and solid-state NMR has also been able to show how the first stages of alpha-synuclein misfolding occur in the presence of lipid membranes ${ }^{83}$. However, it has also been recently demonstrated that despite the appearance of common themes in the structure of amyloid fibrils, different "strains" of fibrils coming from the same protein can be formed, each possessing particular structural properties, different toxicity levels, and in vitro and in vivo 
seeding and propagation characteristics ${ }^{84}$. Future studies are undoubtedly necessary in order to shed light on the nature and role of protein aggregation in neurodegenerative diseases.

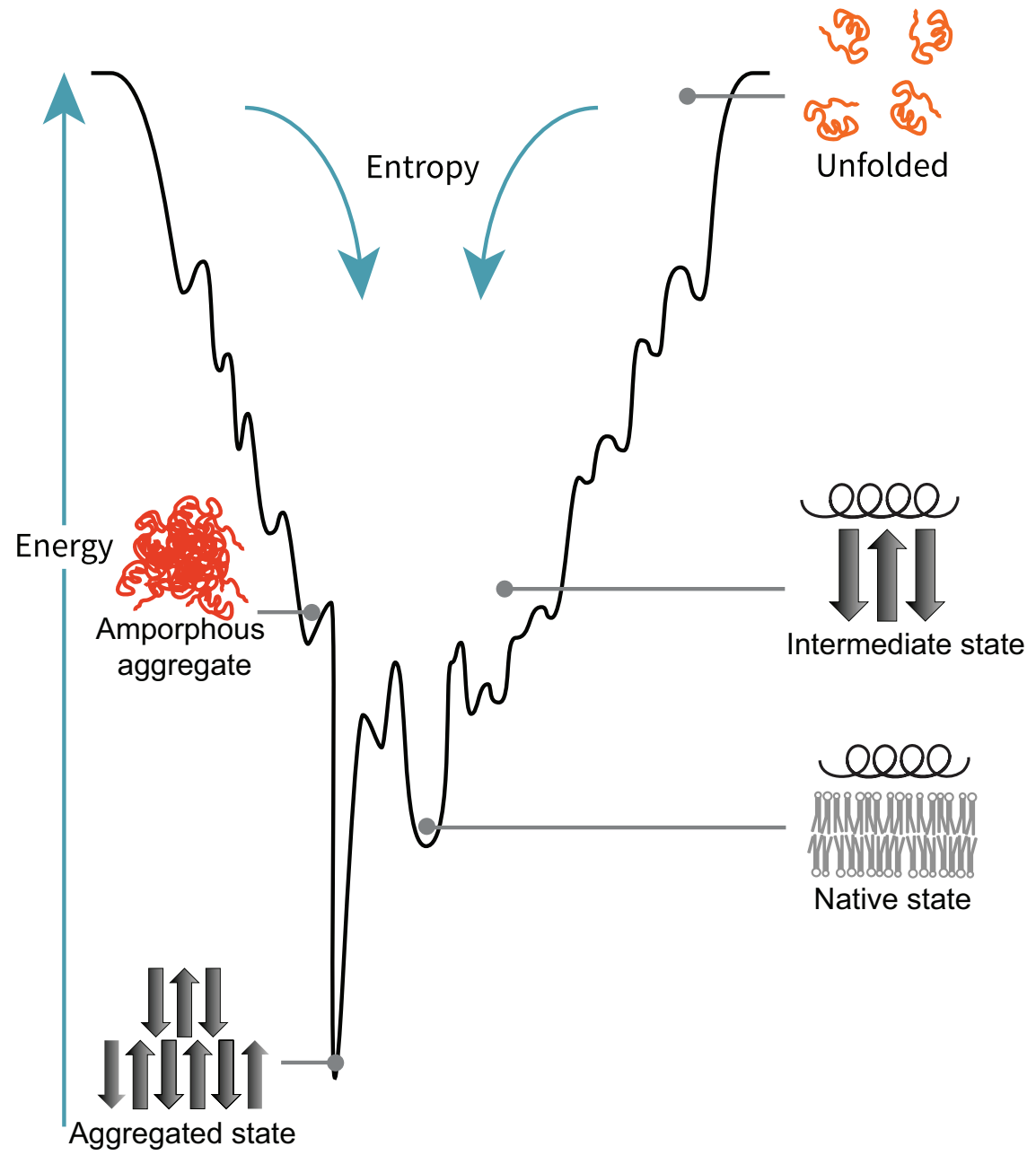

Figure 7. Energy landscape for protein folding. While typically the lowest energy conformation is the functional one, there are other energy levels that can be reached (intermediate state) which can prevent the protein from performing its function (aggregated state).

Another interesting characteristic of alpha-synuclein aggregates (and other neurodegenerative disease-related amyloids) is that it has been recently shown that 
they can spread in a prion-like fashion. Pathogenic forms of alpha-synuclein transmit form cell-to-cell their folding information and "infect" healthy proteins, promoting their misfolding and greatly contributing to the advancement of the disease ${ }^{85,86}$. But what does "prion-like" mean?

\section{The prion paradigm}

Francis Crick coined one of the most important concepts in modern biology in 1956, when he suggested that the course of information encoded within the DNA of a living organism could only follow a defined pathway from DNA, then to RNA and then to proteins. Called "the central dogma of molecular biology", it states that the DNA carries the information not only to perpetuate itself (by means of what we now call replication), but also to synthesize single stranded nucleic acids in the form of RNA (the process that we now call transcription). The newly formed RNA can sometimes be a finished product and serve important cellular functions (such as the ones found in ribosomes), but most of it carries the necessary information to create proteins (the translation process). The protein, however, is a dead end in which the information encoded within it cannot be transferred back to either protein or nucleic acid, and it has no means of self replication (Figure 8$)^{87}$. Nevertheless, even by the time Crick's paper was published in $1970^{87}$, the general knowledge-or lack thereof-of molecular biology, allowed to leave certain avenues open for further clarification. Francis Crick, for example, said that while virtually impossible, the transmission of information from protein to protein and from protein to nucleic acid could represent a pathway for which, at the time, there was simply no evidence whatsoever and, in the very article in which he published his dogma, he mentioned an interesting phenomena reported by Gibbons and Hunter in 1967. There, they describe the "scrapie agent", an element 
devoid of nucleic acids capable of infecting the brain of sheep and "replicating" within $i^{88}$. While scrapie had been known as a sheep disease since the 1930 s, it was until then that the suspicions about a possible violation of the dogma started to take shape. Already believing that the protein was the most likely culprit, Gibbons and Hunter foresaw the impact that this would have on the central dogma of molecular biology: it implied that an agent lacking nucleic acids would indeed be capable of both replicating its information and transmit it and thus the information contained in a protein was not in a "dead end".

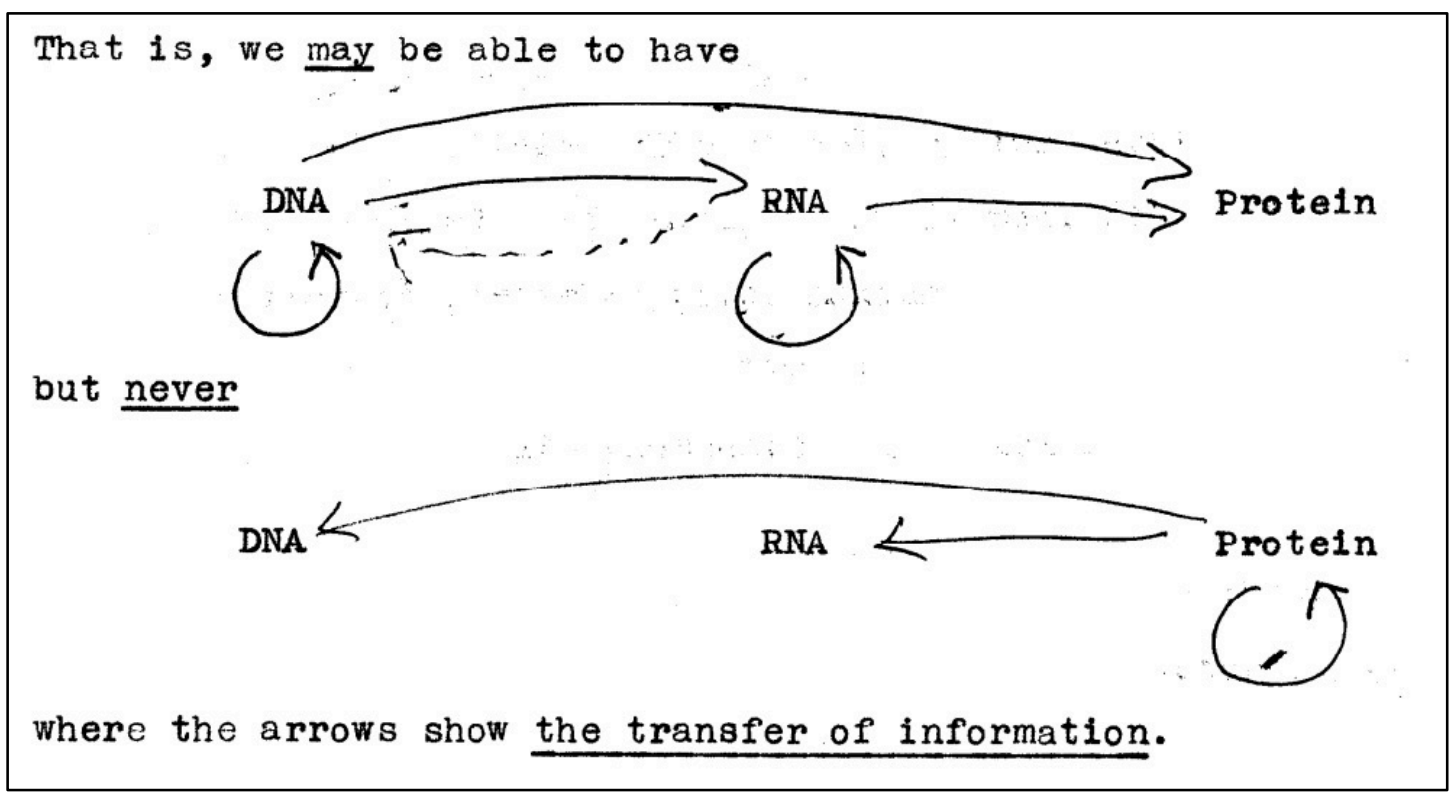

Figure 8. Early draft of the central dogma of molecular biology. Proposed by Francis Crick in $1956^{87}$.

\section{The protein-only hypothesis}

The seminal article that propelled the protein-only hypothesis was published by J.S. Griffith in 1967, where he introduced the idea that the material responsible for disease transmission might be a protein that had the surprising ability to replicate in the body (until then, this and other diseases were wrongly attributed to different kinds of 
viruses, for example), had the capability to infect goats, rats, mice or hamsters ${ }^{89}$. The person in charge of coining the term "prion" was Stanley Prusiner, who published an article in 1982 to denote "a small proteinaceous infectious particle which is resistant to inactivation by most procedures that modify nucleic acids" that was the responsible agent that infected the central nervous system of sheep and goats ${ }^{90}$. Initially facing tough criticisms, his views are now the most accepted pathways through which the prion protein gains its infectivity.

The most important aspect to determine the nature of this new infectious agent was the isolation of the protease-resistant prion protein $\left(\operatorname{PrP}^{\mathrm{res}}\right)$ from the infectious material ${ }^{91}$. Highly purified preparations of $\mathrm{PrP}^{\text {res }}$, in which no other component was detectable, retained their infectivity. In addition, infectivity was convincingly reduced by agents that destroy protein structure and, more importantly, was recognized by antibodies to $\operatorname{PrP}^{92,93}$. Purification of the protein allowed identification of the gene encoding $\operatorname{PrP}^{94}$. PrP mRNA proved to be the product of a single host gene, which is present in the brain of uninfected animals and is constitutively expressed by many cell types. It thus became clear that PrP could potentially exist in at least two alternative forms: the normal cellular prion $\left(\mathrm{PrP}^{\mathrm{C}}\right)$ and the pathological isoform found in scrapie that is resistant to degradation $\left(\mathrm{PrP}^{\mathrm{res}}\right.$ or $\left.\mathrm{PrP}^{\mathrm{sc}}\right)$. Since no evident chemical differences distinguishing these two PrP isoforms have been thoroughly identified, it was proven that the conversion between healthy and disease-promoting PrP involves merely a conformational modification whereby the alpha-helical content of the normal protein is diminished and the beta-sheet content is increased ${ }^{95}$. These structural changes are followed by alterations in other biochemical properties that result in the protease resistance, solubility and the ability to form larger-order aggregates. 
A compelling piece of evidence supporting the protein-only hypothesis came from the laboratory of Charles Weissmann in a study, which determined that mice devoid of PrP are resistant to scrapie infection, neither developing signs of scrapie nor allowing propagation of the disease ${ }^{96}$. A further milestone in support of the prion hypothesis was the finding that the pathological protein catalyzed the cell-free conversion of $\operatorname{PrP}^{\mathrm{C}}$ into $\operatorname{PrP}^{\mathrm{res} 97}$.

\section{Prion protein in humans}

The prion protein is also found in humans and it certainly acquired a prominent role when it was found that misfolded prions were able to "infect" properly folded prions of the host, and even capable of doing this in a species different from the one in which the toxic prion was generated ${ }^{98}$. Indeed kuru, appearing in the Fore people in Papua New Guinea who favored the transmission of a misfolded prion protein with cannibalistic rituals, developed a widespread form of the disease ${ }^{99}$. In the western world, prion disease came into the focus when it was discovered that human consumption of meat coming from cows suffering from bovine spongiform encephalopathy (another form of prion disease) promoted some cases of infection in humans ${ }^{100,101}$. Genetic studies further showed that most, if not all, familial cases of TSE are linked to mutation within the PrP gene ${ }^{6}$. These findings not only provided support for a central role or PrP in disease pathogenesis, but also offered compelling evidence for the protein-only hypothesis, because the genetic disease can be propagated in an infectious way. Interestingly, a TSE-like disease was produced in mice overexpressing PrP genes with point mutations linked to the GSS syndrome ${ }^{102}$. These animals spontaneously developed neurologic dysfunction, spongiform brain degeneration and astrocytic gliosis. 
In humans, PrP is encoded by the PRNP gene as a single copy and it is located on chromosome $20^{103,104}$. Its misfolding from $\operatorname{PrP}^{\mathrm{C}}$ to $\operatorname{PrP}^{\mathrm{res}}$ has been associated with spongiform encephalopathies, such as Creutzfeld-Jakob disease (CJD), variantCreutzfeld-Jakob disease (vCJD), fatal familial insomnia (FFI), GertsmannStraussler-Scheinker syndrome (GSS), kuru, and variable protease-sensitive prionopathy (VPSPr) (reviewed in reference 105). At the pathological level, prion diseases are characterized by vacuolation (spongiform change) in the brain, together with neuronal loss, astrocytosis, and the intracerebral buildup of misfolded PrP (Figure 9) ${ }^{106,107}$. Even though the prevalence of prion-related diseases is fairly low (less than 6 cases per million people between 65 and 79 years of age ${ }^{108}$ ), they are all invariable fatal a few years after diagnosis, and no known cure is available ${ }^{86}$. Prions are not necessarily well known because of the impact its incidence has on the national health budgets (as Alzheimer's or Parkinson's disease do), but because of the very particular mechanisms by which it spreads its toxicity. These mechanisms challenge, as stated at the beginning of this section, some of the most fundamental concepts in molecular biology. Plus, the fact that the disease can sporadically appear (CreutzfeldtJakob disease), or that it can be promoted via inherited mutations present in the PrP gene (familial Creutzfeldt-Jakob disease, familial fatal insomnia, GertsmannSträussler-Scheinker disease), or that it can be acquired by intra and interspecific infections (kuru, variant Creutzfeldt-Jakob disease) makes this a fascinating - yet fearsome - protein. 


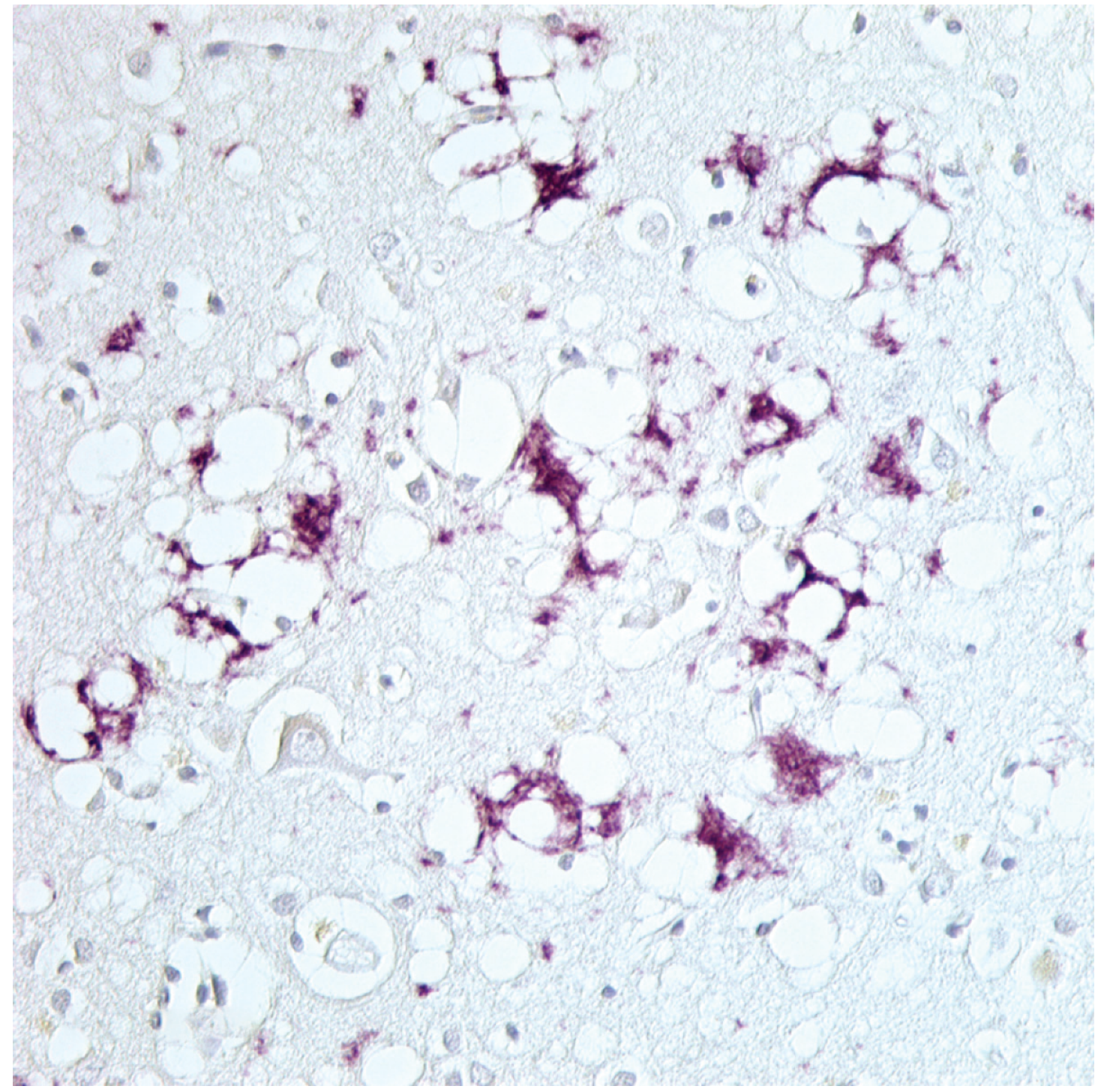

Figure 9. Prion protein aggregates. Immunoreactivity (purple) and spongiform degeneration in the brain of a patient with Creutzfeldt-Jakob disease. From reference 6.

The structure of PrP

The shared characteristic between the three forms of prion diseases (sporadic, inherited, and acquired via infection) is a profound conformational change in the structure of the normal $\operatorname{PrP}^{\mathrm{C}}$ to a pathogenic $\mathrm{PrP}^{\mathrm{Sc} / \mathrm{res}}$. The PrP consists of 240 amino acids with a mature form of 209 (23-231), with a large 40 amino acid-long octarepeat 
region capable of binding copper, two sites of N-linked glycosylation, a disulfide bond, a glycolipid (GPI) anchor and a highly conserved sequence between species which spans from residues 110-136 (Figure 10). The GPI anchor makes most of the $\operatorname{PrP}^{\mathrm{C}}$ to be attached to the plasma membrane in the extracellular space. $\operatorname{PrP}^{\mathrm{C}}$ shows a globular C-terminal domain, in which NMR spectroscopy revealed three well-defined alpha-helices and two small beta-sheets ${ }^{109}$ (blue and yellow stretches, respectively in Figure 10 and Figure 11). The 23-121 N-terminal domain has a mostly intrinsically disordered structure, and it is not depicted in figure $11^{109}$. A disulfide bridge can form between cysteines in the second and third helix and its reduction has been linked to a structural modification that is necessary for the initial formation of fibrils ${ }^{110}$. Notably, the conserved $\mathrm{N}$-terminal sequence among mammalian prions is part of the central core of formed fibrils found in $\mathrm{PrP}^{\mathrm{Sc}}$.

Another interesting property of human prion-related diseases is that homozygosity at the polymorphic position 129 is predominantly present in people with sporadic Creutzfeldt-Jakob disease. While the general population has a 50/50 chance of being either Met/Val, up to $89 \%$ of the CJD patients are either Met/Met or $\mathrm{Val} / \mathrm{Val}$ at this position, suggesting a special role for homology within this region for prion aggregation $^{111}$. 

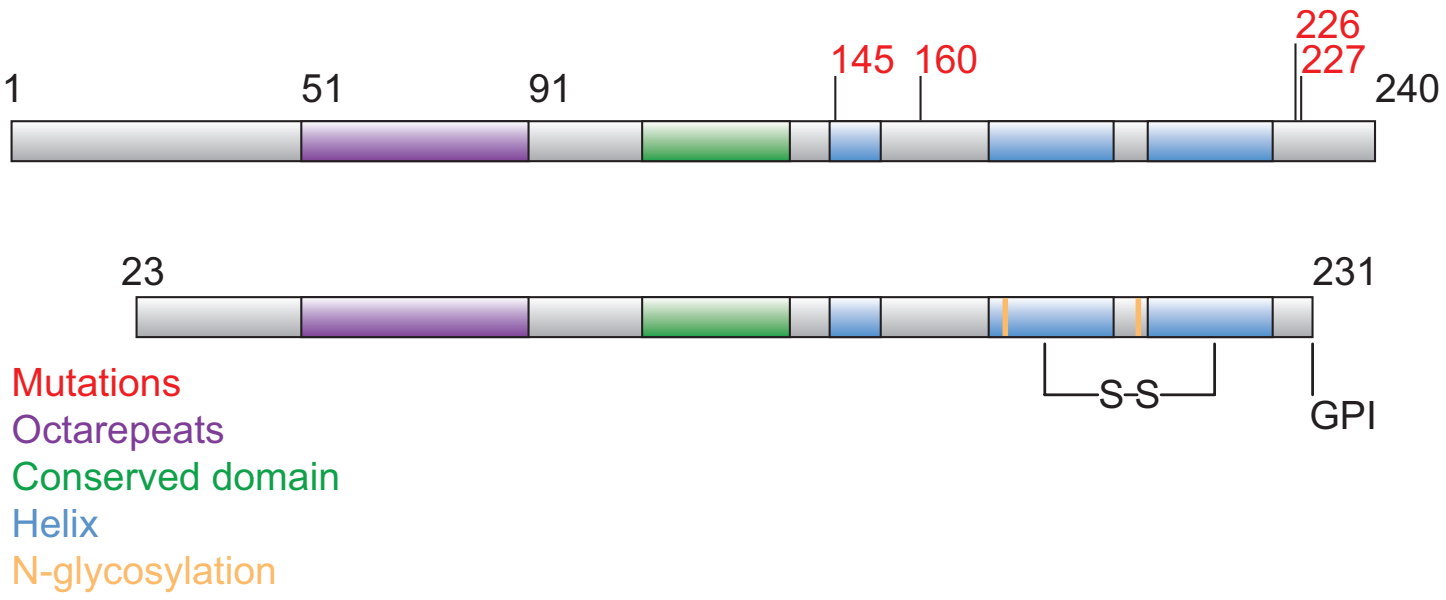

Figure 10. Schematic representation of the main regions and characteristics of the prion protein. The mutation sites in red correspond to inherited forms of prion diseases. Residues 1-22 and 232-240 are cleaved during maturation of the protein.

Similar to most proteins that misfold in neurodegenerative diseases, it is not known whether the cause of such diseases is the formation of the prototypical betasheet-rich fibrils, or toxicity through gain, alteration, or loss of function of the birthing protein. The misfolding from alpha-helix-rich, to beta-sheet-rich is, without a doubt, a hallmark of this process and, therefore, finding ways to inhibit this process represents one of the most coveted avenues in prion protein research. One challenging issue is, however, that the physiological role of $\operatorname{Prp}^{\mathrm{C}}$ is not well understood and many efforts to explain it have produced contrasting results ${ }^{112}$. Some of the putative functions of $\operatorname{PrP}^{\mathrm{C}}$ are related to the central nervous system, because it is precisely there where it is most highly expressed and the prion-related diseases are brain-localized). They include neuronal survival, neurite outgrowth, synapse formation - as well as maintenance and functionality - and formation of myelinated fibers ${ }^{112,113} \cdot \operatorname{PrP}^{\mathrm{C}}$ is also expressed, in many other tissues, indicating that it might have many other functions. Indeed, one of the most accepted roles of $\mathrm{PrP}^{\mathrm{C}}$ is metal homeostasis, because $\operatorname{PrP}$ 
knockout models show a higher sensitivity to metal-derived oxidative stress and higher levels of lipid and protein oxidation ${ }^{114,115}$.

A key aspect of prion biology is that a misfolded prion protein from one species can potentially reach another species' prion protein and promote its misfolding. This is known as the species barrier and, while not universal (sheep prion is not capable of "infecting" human prion, but cow is, for example ${ }^{116}$ ), its understanding will not only provide basic knowledge about the mechanisms governing PrP misfolding (and therefore, ways to interfere with it), but also a more educated approach to the possible dangers that prion diseases in livestock poses to human health.

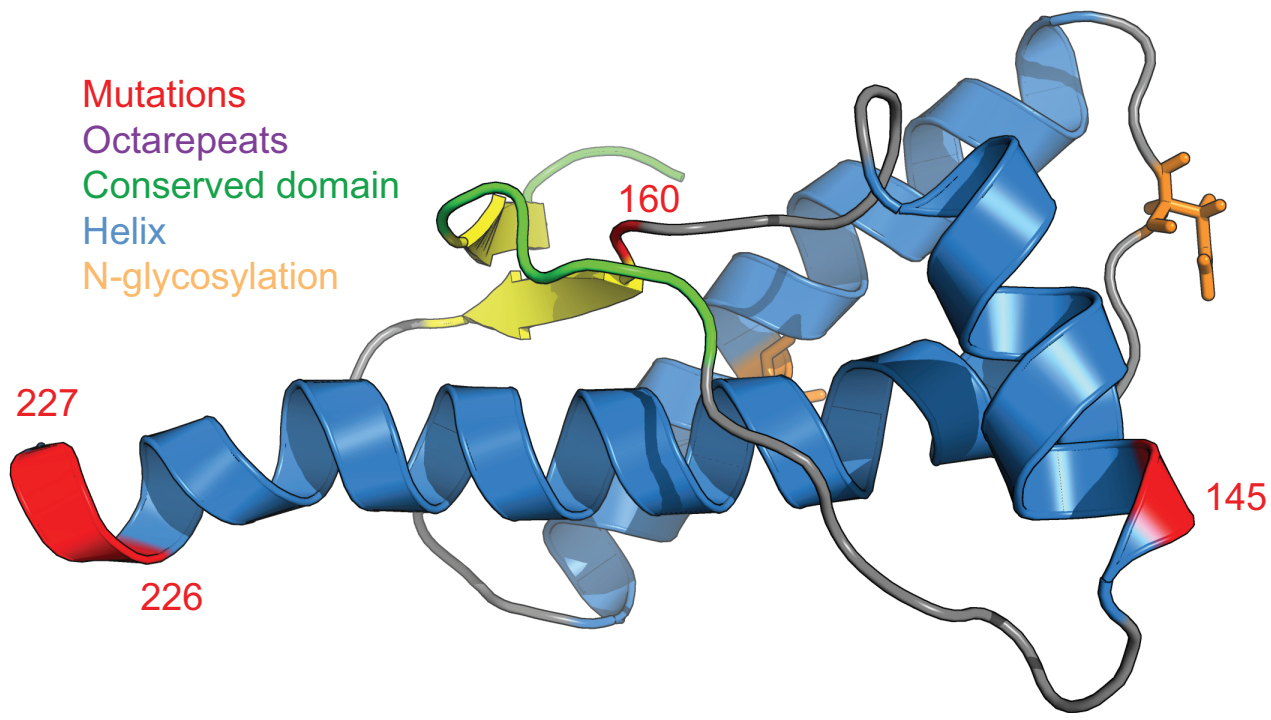

Figure 11. Cartoon of the published three-dimensional structure of the human prion protein ${ }^{109}$. Residues 23-121 are disordered and not presented. The same colorcoding from figure 10 is used. PDB id: 1QM2. 


\section{SIGNIFICANCE}

Whether the aggregation of alpha-synuclein is the driving mechanism by which synucleinopathies arise or its oligomeric and fibrillar forms are just an innocent byproduct of other cellular insults is still a matter of debate. Inhibiting the misfolding of alpha-synuclein remains, regardless, one of the main targets for stopping the disease. A common way to do it is through the screening of small chemical compounds that, under aggregating-prone conditions, keep alpha-synuclein in a monomeric state in solution or, in some cases, in a non-toxic soluble oligomeric state $^{117-120}$. During the development of this thesis, we studied the effects of several known antiamyloidogenic small chemical compounds and tested them in, from our perspective, a more physiological environment: the alpha-helical, vesicle-bound alpha-synuclein. In these studies we found that the mechanisms that govern the misfolding and aggregation of alpha-synuclein vary significantly in the presence and absence of vesicles, and that one of the used compounds, Phthalocyanyne tetrasulfonate (PcTS), inhibits the aggregation of alpha-synuclein by stabilization of its helical vesicle-bound structure thereby preventing its most hydrophobic region to insert into the membrane. We further showed that PcTS inhibits the formation of inclusions and toxicity in a cell model of alpha-synuclein aggregation. To this end, we made use of biophysical and biomolecular techniques that are further described in the methods section. In addition, we determined the role of specific amino acids in the formation of aggregates of an evolutionary conserved fragment of the prion protein. We determined the role of both valine and methionine at the polymorphic position 129 , the role of a highly protected fibrillar core encompassing residues 111-136, and how homology within this specific region is of direct importance for the prion species barrier. 


\section{SELECTED METHODS}

\section{Nuclear magnetic resonance}

Nuclear magnetic resonance spectroscopy is a powerful technique that can be used to investigate the structure, dynamics and chemical kinetics of a wide range of biochemical systems. One of the more powerful attributes of NMR spectroscopy is its ability to detect molecular motion in proteins and other polymers, as well as binding partners and related dynamics. Other methods of detecting molecular motion, such as fluorescence spectroscopy, are limited by the small number of sites that can be probed and the narrow time scale over which the motion can be characterized. The ability to observe and characterize resolved NMR resonance lines from individual atoms provides information on dynamics from a large number of sites within the protein ${ }^{121}$. Though there are many NMR-derived experiments available to assess a wide variety of questions, we have primarily made use of the Heteronuclear Single Quantum Coherence (HSQC) experiment. The HSQC is a highly sensitive two-dimensional NMR experiment, which provides a 2D heteronuclear chemical shift correlation map between directly bonded ${ }^{1} \mathrm{H}$ and X-heteronuclei (commonly ${ }^{13} \mathrm{C}$ and ${ }^{15} \mathrm{~N}$ ). It is widely used because it is based on proton detection, offering high sensitivity. The most substantial technical difference between this experiment and the homonuclear experiments is the use of radio frequency pulses that independently alter the proton or heteronuclear magnetization. Independent excitation of the different spins allows greater flexibility in manipulation of the spins during the experiment (Figure 12). 


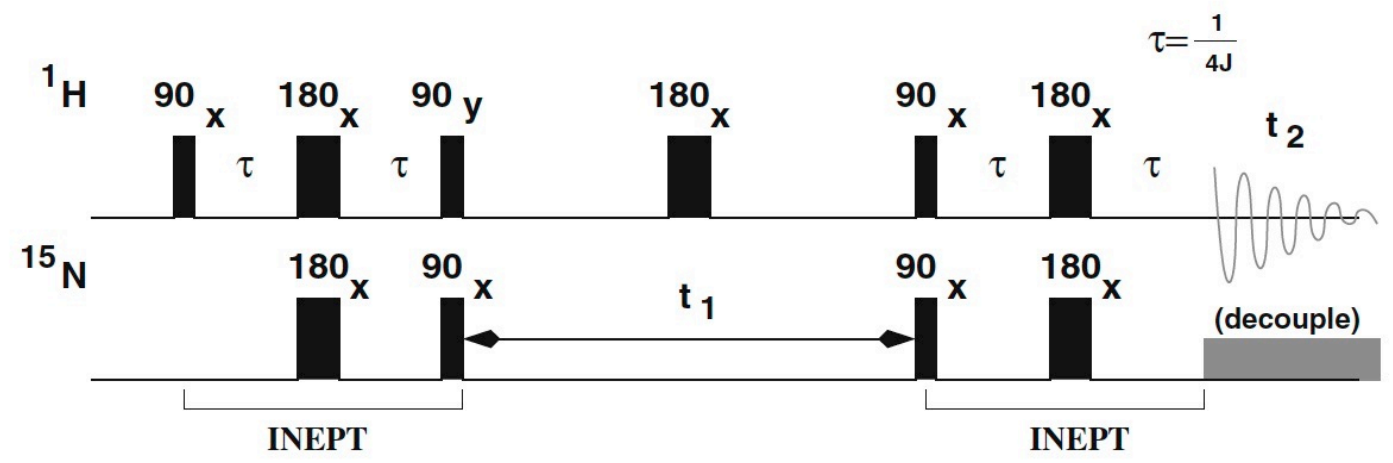

Figure 12. HSQC pulse sequence. The top sets of pulses are applied to the protons and the lower set of the pulses are applied to the heteronuclear spins $\left({ }^{15} \mathrm{~N}\right.$ in this illustration) via a separate radio-frequency channel. Narrow bars correspond to $90 \circ$ pulses and wider bars represent 180 degrees pulses. The delay $\tau$ is nominally set to 1/4J. Polarization transfer periods (INEPT) are labeled and include the simultaneous proton and nitrogen 90 degrees pulse ${ }^{121}$.

The successful use of $2 \mathrm{D}^{1} \mathrm{H}_{-}{ }^{15} \mathrm{~N}$ HSQC is based on the large dispersion of backbone ${ }^{15} \mathrm{~N}$ amide and ${ }^{13} \mathrm{C}$ carbonyl chemical shifts and their dependency on the local environment. Each residue type exhibits a corresponding random coil value, where ${ }^{15} \mathrm{~N}$ random coil values are better dispersed (100-135 ppm) than the ${ }^{1} \mathrm{H}$ values (6.5-9.5 ppm). The chemical shifts of residues in IDPs are indeed close to these random coil values and hence the dispersion is small. Changes in the local environment (because of interaction with small compounds or big vesicles, for example) have profound effects in the place at which the heteronuclei frequencies are found. These differences can be quantified and important information (at a residue-specific resolution) can be thus obtained. Chemical shift perturbation (CSP) is one of the most common techniques to assess binding site, ligand affinity, and even complex structure. 


\section{Circular dichroism}

Circular dichroism (CD) is an excellent method for rapidly evaluating the secondary structure, folding and binding properties of proteins. Circular dichroism is defined as the unequal absorption of left-handed and right-handed circularly polarized light. A beam of light has time dependent electric and magnetic fields associated with it. If the light is polarized by passing through suitable prisms or filters its electric field, E, will oscillate sinusoidally in a single plane. When viewed from the front, the sinusoidal wave can be visualized as the resultant of two vectors of equal length, which trace out circles, one which rotates clockwise (ER) and the other which rotates counterclockwise (EL). The two circularly polarized waves have physical existence. The waves are 90 degrees out of phase with each other and can be separated using a variety of prisms or electronic devices which utilize Pockel's effect. When asymmetric molecules interact with light, they may absorb right and left handed circularly polarized light to different extents (hence the term circular dichroism) and also have different indices of refraction for the two waves. The result is that the plane of the light wave is rotated and that the addition of the ER and EL vectors results in a vector that traces out an ellipse and the light is said to be elliptically polarized. CD is reported either in units of $\Delta \mathrm{E}$, the difference in absorbance of ER and EL by an asymmetric molecule, or in degrees ellipticity, which is defined as the angle whose tangent is the ratio of the minor to the major axis of the ellipse. CD is an excellent method of determining the secondary structure of proteins. When the chromophores of the amides of the polypeptide backbone of proteins are aligned in arrays, their optical transitions are shifted or split into multiple transitions due to "exciton" interactions. The result is that different structural elements have characteristic CD spectra. For example, $\alpha$-helical proteins have negative bands at $222 \mathrm{~nm}$ and $208 \mathrm{~nm}$ 
and a positive band at $193 \mathrm{~nm}$. Proteins with well-defined antiparallel $\beta$-pleated sheets ( $\beta$-helices) have negative bands at $218 \mathrm{~nm}$ and positive bands at $195 \mathrm{~nm}$, while disordered proteins have very low ellipticity above $210 \mathrm{~nm}$ and negative bands near $195 \mathrm{~nm}^{122}$. The absorption within these ranges can be then computed using different methods, and a percentage of $\alpha$-helix vs $\beta$-sheets vs random coil can be obtained ${ }^{123}$.

\section{Thioflavin T fluorescence}

Thioflavin $\mathrm{T}$ is a benzothiazole dye that exhibits enhanced fluorescence upon binding to amyloid fibrils and is commonly used to diagnose amyloid fibrils, both ex vivo and in vitro. When it binds to beta sheet-rich structures, such as those in amyloid aggregates, the dye displays enhanced fluorescence and a characteristic red shift of its emission spectrum. This change in fluorescent behavior can be caused by many factors that affect the excited state charge distribution of thioflavin $\mathrm{T}$, including binding to a rigid, highly-ordered amyloid structure, or to specific chemical interactions with a protein ${ }^{124}$. 


\section{REFERENCES}

1. http://www.aoa.gov

2. Olshansky, S. J. \& Carnes, B. A. Ageing and health. The Lancet 375, 25 (2010).

3. Finkel, T. Radical medicine: treating ageing to cure disease. Nat Rev Mol Cell Biol 6, 971-976 (2005).

4. $\quad$ Christensen, K., Doblhammer, G., Rau, R. \& Vaupel, J. W. Ageing populations: the challenges ahead. The Lancet 374, 1196-1208 (2009).

5. High, K. P. Infrastructure and resources for an aging population: embracing complexity in translational research. Transl Res 163, 446-455 (2014).

6. Walker, L. C. \& Jucker, M. Neurodegenerative diseases: expanding the prion concept. Annu. Rev. Neurosci. 38, 87-103 (2015).

7. Chiti, F. \& Dobson, C. M. Protein misfolding, functional amyloid, and human disease. Annu. Rev. Biochem. 75, 333-366 (2006).

8. Caughey, B. \& Lansbury, P. T., Jr. Protofibrils, pores, fibrils, and neurodegeneration: separating the responsible protein aggregates from the innocent bystanders. Annu. Rev. Neurosci. 26, 267-298 (2003).

9. Morriss-Andrews, A. \& Shea, J.-E. Computational studies of protein aggregation: methods and applications. Annu Rev Phys Chem 66, 643-666 (2015).

10. Swietnicki, W., Morillas, M., Chen, S. G. \& Gambetti, P. Aggregation and fibrillization of the recombinant human prion protein huPrP90-231.

Biochemistry (2000). doi:10.1021/bi991967m

11. Conway, K. A. et al. Acceleration of oligomerization, not fibrillization, is a shared property of both alpha-synuclein mutations linked to early-onset Parkinson's disease: implications for pathogenesis and therapy. Proc. Natl. Acad. Sci. U.S.A. 97, 571-576 (2000).

12. Uversky, V. N., Li, J. \& Fink, A. L. Evidence for a partially folded intermediate in alpha-synuclein fibril formation. J. Biol. Chem. 276, 1073710744 (2001).

13. Bertoncini, C. W., Fernandez, C. O. \& Griesinger, C. Familial mutants of $\alpha$ synuclein with increased neurotoxicity have a destabilized conformation. $J$ Biol Chem (2005). doi:10.1074/jbc.C500288200

14. Karpinar, D. P., Balija, M., Kügler, S. \& Opazo, F. Pre - fibrillar $\alpha$-synuclein variants with impaired $\beta$-structure increase neurotoxicity in Parkinson's disease models. The EMBO (2009). doi:10.1038/emboj.2009.257

15. Lashuel, H. A., Petre, B. M., Wall, J. \& Simon, M. $\alpha$-Synuclein, especially the Parkinson's disease-associated mutants, forms pore-like annular and tubular protofibrils. J Biol Chem (2002). doi:10.1016/S0022-2836(02)007350

16. Parkinson, J. An Essay on the Shaking Palsy. (1817).

17. Catala, M. \& Poirier, J. Georges Marinesco (1863-1938): neurologist, neurohistologist and neuropathologist. Rom J Morphol Embryol (2012).

18. Goedert, M., Spillantini, M. G., Del Tredici, K. \& Braak, H. 100 years of Lewy pathology. Nature reviews. Neurology 9, 13-24 (2013).

19. Hornykiewicz, O. A brief history of levodopa. J. Neurol. 257, S249-52 (2010).

20. Abbott, A. Levodopa: the story so far. Nature 466, S6-7 (2010).

21. Moore, D. J., West, A. B., Dawson, V. L. \& Dawson, T. M. Molecular 
pathophysiology of Parkinson's disease. Annu. Rev. Neurosci. 28, 57-87 (2005).

22. Shulman, J. M., De Jager, P. L. \& Feany, M. B. Parkinson's Disease: Genetics and Pathogenesis. Annu. Rev. Pathol. Mech. Dis. 6, 193-222 (2011).

23. Braak, H., Del Tredici, K., Rüb, U. \& de Vos, R. Staging of brain pathology related to sporadic Parkinson's disease. Neurobiol dis (2003).

24. Lundqvist, C. Continuous levodopa for advanced Parkinson's disease. Neuropsychiatr. Dis. (2007).

25. Zucca, F. A. et al. Interactions of iron, dopamine and neuromelanin pathways in brain aging and Parkinson's disease. Prog. Neurobiol. (2015). doi:10.1016/j.pneurobio.2015.09.012

26. Cookson, M. R. The biochemistry of Parkinson's disease. Annu. Rev. Biochem. 74, 29-52 (2005).

27. Lees, A. J., Hardy, J. \& Revesz, T. Parkinson's disease. Lancet 373, 20552066 (2009).

28. Goedert, M. Alpha-synuclein and neurodegenerative diseases. Nat. Rev. Neurosci. (2001).

29. Dickson, D. W., Braak, H., Duda, J. E. \& Duyckaerts, C. Neuropathological assessment of Parkinson's disease: refining the diagnostic criteria. The Lancet (2009). doi:10.1016/S1474-4422(09)70238-8

30. Shults, C. W. Lewy bodies. Proc. Natl. Acad. Sci. U.S.A. 103, 1661-1668 (2006).

31. Kozlowski, H., Luczkowski, M. \& Remelli, M. Copper, zinc and iron in neurodegenerative diseases (Alzheimer's, Parkinson's and prion diseases). Coord Chem (2012). doi:10.1016/j.ccr.2012.03.013

32. Spillantini, M. G., Crowther, R. A. \& Jakes, R. $\alpha$-Synuclein in filamentous inclusions of Lewy bodies from Parkinson's disease and dementia with Lewy bodies. Proc. Natl. Acad. Sci. U.S.A (1998).

33. Bertoncini, C. W. et al. Release of long-range tertiary interactions potentiates aggregation of natively unstructured alpha-synuclein. Proc. Natl. Acad. Sci. U.S.A. 102, 1430-1435 (2005).

34. Chen, S. W. et al. Structural characterization of toxic oligomers that are kinetically trapped during $\alpha$-synuclein fibril formation. Proc. Natl. Acad. Sci. U.S.A. 112, E1994-2003 (2015).

35. Conway, K. A., Harper, J. D. \& Lansbury, P. T., Jr. Accelerated in vitro fibril formation by a mutant alpha-synuclein linked to early-onset Parkinson disease. Nat. Med. 4, 1318-1320 (1998).

36. Maroteaux, L., Campanelli, J. T. \& Scheller, R. H. Synuclein: a neuronspecific protein localized to the nucleus and presynaptic nerve terminal. $J$. Neurosci. 8, 2804-2815 (1988).

37. Jakes, R., Spillantini, M. G. \& Goedert, M. Identification of two distinct synucleins from human brain. FEBS Lett 345, 27-32 (1994).

38. Nakajo, S., Tsukada, K., Omata, K., Nakamura, Y. \& Nakaya, K. A new brain-specific 14-kDa protein is a phosphoprotein. Its complete amino acid sequence and evidence for phosphorylation. Eur. J. Biochem. 217, 1057-1063 (1993).

39. Clayton, D. F. \& George, J. M. The synucleins: a family of proteins involved in synaptic function, plasticity, neurodegeneration and disease. Trends Neurosci. 21, 249-254 (1998).

40. George, J. M., Jin, H., Woods, W. S. \& Clayton, D. F. Characterization of a 
novel protein regulated during the critical period for song learning in the zebra finch. Neuron 15, 361-372 (1995).

41. Weinreb, P. H., Zhen, W., Poon, A. W., Conway, K. A. \& Lansbury, P. T., Jr. NACP, a protein implicated in Alzheimer's disease and learning, is natively unfolded. Biochemistry 35, 13709-13715 (1996).

42. Polymeropoulos, M. H. et al. Mutation in the $\alpha$-synuclein gene identified in families with Parkinson's disease. Science 276, 2045-2047 (1997).

43. Spillantini, M. G. et al. Alpha-synuclein in Lewy bodies. Nature 388, 839840 (1997).

44. Krüger, R. et al. Ala30Pro mutation in the gene encoding alpha-synuclein in Parkinson's disease. Nat. Genet. 18, 106-108 (1998).

45. Zarranz, J. J. et al. The new mutation, E46K, of alpha-synuclein causes Parkinson and Lewy body dementia. Ann. Neurol. 55, 164-173 (2004).

46. Proukakis, C., Houlden, H. \& Schapira, A. H. Somatic alpha-synuclein mutations in Parkinson's disease: Hypothesis and preliminary data. Mov. Disord. (2013). doi:10.1002/mds.25502

47. Singleton, A. B. et al. alpha-Synuclein locus triplication causes Parkinson's disease. Science 302, 841 (2003).

48. Lesage, S., Anheim, M., Letournel, F. \& Bousset, L. G51D $\alpha$-synuclein mutation causes a novel Parkinsonian-pyramidal syndrome. Ann Neurol. (2013). doi:10.1002/ana.23894

49. Breydo, L., Wu, J. W. \& Uversky, V. N. $\alpha$-Synuclein misfolding and Parkinson's disease. BBA - Molecular Basis of Disease 1-25 (2011). doi:10.1016/j.bbadis.2011.10.002

50. Chen, X. et al. The human NACP/alpha-synuclein gene: chromosome assignment to 4q21.3-q22 and TaqI RFLP analysis. Genomics 26, 425-427 (1995).

51. Auluck, P. K., Caraveo, G. \& Lindquist, S. $\alpha$-Synuclein: Membrane Interactions and Toxicity in Parkinson's Disease. Annu. Rev. Cell Dev. Biol. 26, 211-233 (2010).

52. Bendor, J. T., Logan, T. P. \& Edwards, R. H. The function of $\alpha$-synuclein. Neuron 79, 1044-1066 (2013).

53. Chartier-Harlin, M. C., Kachergus, J. \& Roumier, C. $\alpha$-Synuclein locus duplication as a cause of familial Parkinson's disease. The Lancet (2004).

54. Fujiwara, H. et al. alpha-Synuclein is phosphorylated in synucleinopathy lesions. Nat. Cell Biol. 4, 160-164 (2002).

55. Burre, J. et al. Properties of native brain $\alpha$-synuclein. Nature 498, E4-6discussion E6-7 (2013).

56. Davidson, W. S., Jonas, A., Clayton, D. F. \& George, J. M. Stabilization of alpha-synuclein secondary structure upon binding to synthetic membranes. $J$. Biol. Chem. 273, 9443-9449 (1998).

57. Ulmer, T. S., Bax, A., Cole, N. B. \& Nussbaum, R. L. Structure and dynamics of micelle-bound human alpha-synuclein. J. Biol. Chem. 280, 9595-9603 (2005).

58. Lokappa, S. B. \& Ulmer, T. S. Alpha-synuclein populates both elongated and broken helix states on small unilamellar vesicles. J. Biol. Chem. 286, 2145021457 (2011).

59. Eliezer, D., Kutluay, E., Bussell, R. \& Browne, G. Conformational properties of alpha-synuclein in its free and lipid-associated states. Journal of Molecular Biology 307, 1061-1073 (2001). 
60. Bartels, T., Choi, J. G. \& Selkoe, D. J. $\alpha$-Synuclein occurs physiologically as a helically folded tetramer that resists aggregation. Nature 477, 107-110 (2011).

61. Wang, W. et al. A soluble $\alpha$-synuclein construct forms a dynamic tetramer. Proc. Natl. Acad. Sci. U.S.A. 108, 17797-17802 (2011).

62. Lewis, S. Synaptic plasticity: A key player in presynaptic plasticity. Nature (2011). doi:10.1038/nrn3108

63. Iwai, A. et al. The precursor protein of non-A beta component of Alzheimer's disease amyloid is a presynaptic protein of the central nervous system. Neuron 14, 467-475 (1995).

64. Payton, J. E., Perrin, R. J., Clayton, D. F. \& George, J. M. Protein-protein interactions of alpha-synuclein in brain homogenates and transfected cells. Brain Res. Mol. Brain Res. 95, 138-145 (2001).

65. Fortin, D. L. et al. Lipid rafts mediate the synaptic localization of alphasynuclein. J. Neurosci. 24, 6715-6723 (2004).

66. Golovko, M. Y. et al. Alpha-synuclein gene deletion decreases brain palmitate uptake and alters the palmitate metabolism in the absence of alphasynuclein palmitate binding. Biochemistry 44, 8251-8259 (2005).

67. Abeliovich, A., Schmitz, Y., Fariñas, I. \& Choi-Lundberg, D. Mice lacking $\alpha-$ synuclein display functional deficits in the nigrostriatal dopamine system. Neuron (2000).

68. Greten-Harrison, B. et al. $\alpha \beta \gamma$-Synuclein triple knockout mice reveal agedependent neuronal dysfunction. Proc. Natl. Acad. Sci. U.S.A. 107, 1957319578 (2010).

69. Burre, J. et al. Alpha-synuclein promotes SNARE-complex assembly in vivo and in vitro. Science 329, 1663-1667 (2010).

70. Sharma, M., Burre, J. \& Südhof, T. C. CSP $\alpha$ promotes SNARE-complex assembly by chaperoning SNAP-25 during synaptic activity. Nat. Cell Biol. 13, 30-39 (2011).

71. Lee, H. J., Khoshaghideh, F. \& Lee, S. Impairment of microtubule dependent trafficking by overexpression of $\alpha$-synuclein. Eur J Neurosci (2006). doi:10.1111/j.1460-9568.2006.05210.x

72. Chen, R., Wislet-Gendebien, S. \& Samuel, F. $\alpha$-Synuclein membrane association is regulated by the Rab3a recycling machinery and presynaptic activity. J. Biol. Chem. (2013).

73. Yin, G. et al. $\alpha$-Synuclein interacts with the switch region of Rab8a in a Ser129 phosphorylation-dependent manner. Neurobiol. Dis. (2014). doi:10.1016/j.nbd.2014.06.018

74. Engelender, S., Kaminsky, Z., Guo, X. \& Sharp, A. H. Synphilin-1 associates with alpha-synuclein and promotes the formation of cytosolic inclusions. Nat. Genet. (1999).

75. Smith, W. W. et al. Synphilin-1 attenuates neuronal degeneration in the A53T -synuclein transgenic mouse model. Hum Mol Gen 19, 2087-2098 (2010).

76. Hernández-Vargas, R. et al. Synphilin suppresses $\alpha$-synuclein neurotoxicity in a Parkinson's disease Drosophila model. Genesis 49, 392-402 (2011).

77. Xie, Y.-Y. et al. Interaction with synphilin-1 promotes inclusion formation of alpha-synuclein: mechanistic insights and pathological implication. The FASEB Journal 24, 196-205 (2010).

78. Dobson, C. M. Protein folding and misfolding. Nature 426, 884-890 (2003). 
79. Hong, D.-P., Fink, A. L. \& Uversky, V. N. Structural Characteristics of $\alpha-$ Synuclein Oligomers Stabilized by the Flavonoid Baicalein. Journal of Molecular Biology 383, 214-223 (2008).

80. Kim, H.-Y. et al. Structural properties of pore-forming oligomers of alphasynuclein. J. Am. Chem. Soc. 131, 17482-17489 (2009).

81. Sawaya, M. R. et al. Atomic structures of amyloid cross-beta spines reveal varied steric zippers. Nature 447, 453-457 (2007).

82. Rodriguez, J. A. et al. Structure of the toxic core of $\alpha$-synuclein from invisible crystals. Nature 525, 486-490 (2015).

83. Comellas, G., Lemkau, L. R., Zhou, D. H., George, J. M. \& Rienstra, C. M. Structural Intermediates during $\alpha$-Synuclein Fibrillogenesis on Phospholipid Vesicles. J. Am. Chem. Soc. 134, 5090-5099 (2012).

84. Bousset, L. et al. Structural and functional characterization of two alphasynuclein strains. Nat Commun 4, 2575 (2013).

85. Brundin, P., Melki, R. \& Kopito, R. Prion-like transmission of protein aggregates in neurodegenerative diseases. Nat Rev Mol Cell Biol 11, 301-307 (2010).

86. Prusiner, S. B. Cell biology. A unifying role for prions in neurodegenerative diseases. Science 336, 1511-1513 (2012).

87. Crick, F. Central dogma of molecular biology. Nature 227, 561-563 (1970).

88. Gibbons, R. A. \& Hunter, G. D. Nature of the scrapie agent. Nature 215, 1041-1043 (1967).

89. Griffith, J. S. Self-replication and scrapie. Nature 215, 1043-1044 (1967).

90. Prusiner, S. B. Novel proteinaceous infectious particles cause scrapie. Science 216, 136-144 (1982).

91. Bolton, D. C., McKinley, M. P. \& Prusiner, S. B. Identification of a protein that purifies with the scrapie prion. Science 218, 1309-1311 (1982).

92. McKinley, M. P., Groth, D. \& Prusiner, S. B. Immunoaffinity purification and neutralization of scrapie prion infectivity. in (1988).

93. Peretz, D. et al. Antibodies inhibit prion propagation and clear cell cultures of prion infectivity. Nature 412, 739-743 (2001).

94. Chesebro, B. et al. Identification of scrapie prion protein-specific mRNA in scrapie-infected and uninfected brain. Nature 315, 331-333 (1985).

95. Pan, K. M., Baldwin, M. \& Nguyen, J. Conversion of alpha-helices into betasheets features in the formation of the scrapie prion proteins. in (1993).

96. Büeler, H. et al. Mice devoid of PrP are resistant to scrapie. Cell 73, 13391347 (1993).

97. Kocisko, D. A. et al. Cell-free formation of protease-resistant prion protein. Nature 370, 471-474 (1994).

98. Prusiner, S. B., Scott, M. R., DeArmond, S. J. \& Cohen, F. E. Prion protein biology. Cell 93, 337-348 (1998).

99. Hotchin, J. Kuru as a persisting tolerated infection. The Lancet 2, 28-31 (1966).

100. Will, R. G. et al. A new variant of Creutzfeldt-Jakob disease in the UK. The Lancet 347, 921-925 (1996).

101. Dealler, S. \& Lacey, R. W. Bovine spongiform encephalopathy and man. The Lancet 337, 173-174 (1991).

102. Hsiao, K. K., Scott, M., Foster, D., Groth, D. F. \& DeArmond, S. J. Spontaneous neurodegeneration in transgenic mice with mutant prion protein. Science (1990). 
103. Ciric, D. \& Rezaei, H. Biochemical insight into the prion protein family. Front Cell Dev Biol 3, 5 (2015).

104. Sparkes, R. S. et al. Assignment of the human and mouse prion protein genes to homologous chromosomes. Proc. Natl. Acad. Sci. U.S.A. 83, 7358-7362 (1986).

105. Safar, J. G. Molecular pathogenesis of sporadic prion diseases in man. Prion 6, 108-115 (2012).

106. Parchi, P. et al. Classification of sporadic Creutzfeldt-Jakob disease based on molecular and phenotypic analysis of 300 subjects. Ann. Neurol. 46, 224-233 (1999).

107. Cali, I. et al. Classification of sporadic Creutzfeldt-Jakob disease revisited. Brain 129, 2266-2277 (2006).

108. Holman, R. C., Belay, E. D., Christensen, K. Y. \& Maddox, R. A. Human prion diseases in the United States. PLOS ONE (2010). doi:10.1371/journal.pone.0008521.t003

109. Zahn, R. et al. NMR solution structure of the human prion protein. Proc. Natl. Acad. Sci. U.S.A. 97, 145-150 (2000).

110. Jackson, G. S. et al. Reversible conversion of monomeric human prion protein between native and fibrilogenic conformations. Science 283, 19351937 (1999).

111. Heinemann, U. et al. Creutzfeldt-Jakob disease in Germany: a prospective 12-year surveillance. Brain 130, 1350-1359 (2007).

112. Gasperini, L. \& Legname, G. Prion protein and aging. Front Cell Dev Biol 2, 44 (2014).

113. Aguzzi, A., Baumann, F. \& Bremer, J. The prion's elusive reason for being. Annu. Rev. Neurosci. 31, 439-477 (2008).

114. Wong, B. S. et al. Increased levels of oxidative stress markers detected in the brains of mice devoid of prion protein. Journal of Neurochemistry 76, 565572 (2001).

115. Rachidi, W. et al. Expression of prion protein increases cellular copper binding and antioxidant enzyme activities but not copper delivery. J. Biol. Chem. 278, 9064-9072 (2003).

116. Castilla, J. et al. Crossing the species barrier by $\operatorname{PrP}(\mathrm{Sc})$ replication in vitro generates unique infectious prions. Cell 134, 757-768 (2008).

117. Ehrnhoefer, D. E. et al. EGCG redirects amyloidogenic polypeptides into unstructured, off-pathway oligomers. Nat. Struct. Mol. Biol. 15, 558-566 (2008).

118. Zhu, M. et al. The flavonoid baicalein inhibits fibrillation of alpha-synuclein and disaggregates existing fibrils. J. Biol. Chem. 279, 26846-26857 (2004).

119. Hafner-Bratkovič, I., Gašperšič, J., Šmid, L. M., Bresjanac, M. \& Jerala, R. Curcumin binds to the $\alpha$-helical intermediate and to the amyloid form of prion protein - a new mechanism for the inhibition of PrPSc accumulation. Journal of Neurochemistry 104, 1553-1564 (2007).

120. Braga, C. A. et al. The Anti-Parkinsonian Drug Selegiline Delays the Nucleation Phase of $\alpha$-Synuclein Aggregation Leading to the Formation of Nontoxic Species. Journal of Molecular Biology 405, 254-273 (2011).

121. Rule, G. S. \& Hitchens, T. K. Fundamentals of protein NMR spectroscopy. (2006).

122. Greenfield, N. J. Using circular dichroism spectra to estimate protein secondary structure. Nat Protoc 1, 2876-2890 (2006). 
123. Whitmore, L. \& Wallace, B. A. Protein secondary structure analyses from circular dichroism spectroscopy: Methods and reference databases.

Biopolymers 89, 392-400 (2008).

124. Khurana, R. et al. Mechanism of thioflavin T binding to amyloid fibrils. $J$. Struct. Biol. 151, 229-238 (2005). 


\section{APPENDIX}

\section{The protonation state of Histidine 111 critically influences the aggregation of}

\section{the evolutionary conserved, central region of the human prion protein}

As part of this thesis we demonstrated that within a peptide, which comprises the evolutionary most conserved region of the human prion protein (residues 108-143), the polymorphic residue at position 129 plays a critical role for aggregation. Within this region, residues 111-137 form the most solvent-protected segment in amyloid fibrils of humPrP(108-143), with residue 129 deeply buried inside the fibril core ${ }^{1}$. Here we show that histidine 111, which marks the C-terminal end of the solventprotected fibril core, and serine 135 , at the N-terminal end of the fibrillar core, are in close spatial proximity in amyloid fibrils of humPrP(108-143). We further show that the protonation state of histidine 111 critically influences the aggregation of humPrP(108-143). In addition, we probed the importance of the solvent-protected fibril core for the so-called species barrier, an important aspect of PrP biology. Our studies suggested that sequence homology within the solvent-protected core of amyloid fibrils of humPrP(108-143) is essential for an efficient seeding of aggregation between prions of different species.

\section{Materials and methods}

Variants of humPrP(108-143) with purity exceeding 95\% were obtained from EZBiolab. Variants of lyophilized humPrP(108-143) were dissolved at a concentration of $0.35 \mathrm{mM}$ with ice-cold $25 \mathrm{mM}$ Tris buffer, $\mathrm{pH} 7.5,0.02 \%$ sodium azide and dialyzed overnight against $500 \mathrm{ml}$ of $25 \mathrm{mM}$ Tris, $\mathrm{pH} 7.5,0.02 \%$ sodium azide at $4{ }^{\circ} \mathrm{C}$ in a $500-1000$ Da molecular mass cut-off dialysis membrane. The 
peptide was filtered through a $0.22 \mu \mathrm{m}$ Millipore filter. Concentration was adjusted to $0.15 \mathrm{mM}$ in a $500 \mu \mathrm{l}$ final volume, and the solution was incubated at $12{ }^{\circ} \mathrm{C}$ in low binding protein 1.5-ml Eppendorf tubes. After different incubation times, a $20 \mu 1$ aliquot of the sample was mixed with $1 \mathrm{ml}$ of the thioflavin $\mathrm{T}$ assay solution $(0.1 \mathrm{mM}$ thioflavin $\mathrm{T}$ in $100 \mathrm{mM} \mathrm{NaH}{ }_{2} \mathrm{PO}_{4}, 140 \mathrm{mM} \mathrm{NaCl}, \mathrm{pH} 8.5$ ), incubated $5 \mathrm{~min}$ at room temperature and transferred to a 10-mm cuvette. The fluorescence emission was measured between 460 and $600 \mathrm{~nm}$ on a Varian Cary Eclipse fluorescence spectrophotometer (Agilent Technologies) with excitation at $442 \mathrm{~nm}$ at $20{ }^{\circ} \mathrm{C}$. To have a second measure for aggregate formation of different variants of humPrP(108143), aggregates were collected after 2.7 and $24 \mathrm{~h}$ of incubation by centrifugation at $16,000 \mathrm{~g}$ for 30 minutes at $4{ }^{\circ} \mathrm{C}$. Subsequently, the pellet was resuspended in $100 \mu \mathrm{l}$ of $25 \mathrm{mM}$ Tris $\mathrm{HCl}, \mathrm{pH} 7.5$, and diluted in a $1: 5$ ratio in $7.5 \mathrm{M}$ guanidine hydrochloride, $25 \mathrm{mM} \mathrm{NaH2} \mathrm{PO} 4$, $\mathrm{pH}$ 6.5. Peptide concentrations were determined by UV absorption at $280 \mathrm{~nm}$.

\section{Results and discussion}

Histidine 111 and 140 have similar pKa values in the monomeric, unfolded state of humPrP(108-143)

humPrP(108-143) contains two histidines at positions 111 and 140. According to NMR-based H/D exchange measurements and hydroxyl radical probing detected by mass spectrometry, histidine 111 is located inside the solvent-protected fibril core, in contrast to histidine $140^{1}$ (Figure 13). In addition, a previously generated model of amyloid fibrils of humPrP(108-143), which was generated on the basis of solid-state 
NMR chemical shifts ${ }^{2}$, predicted histidine 111 and serine 135 to be in close proximity.

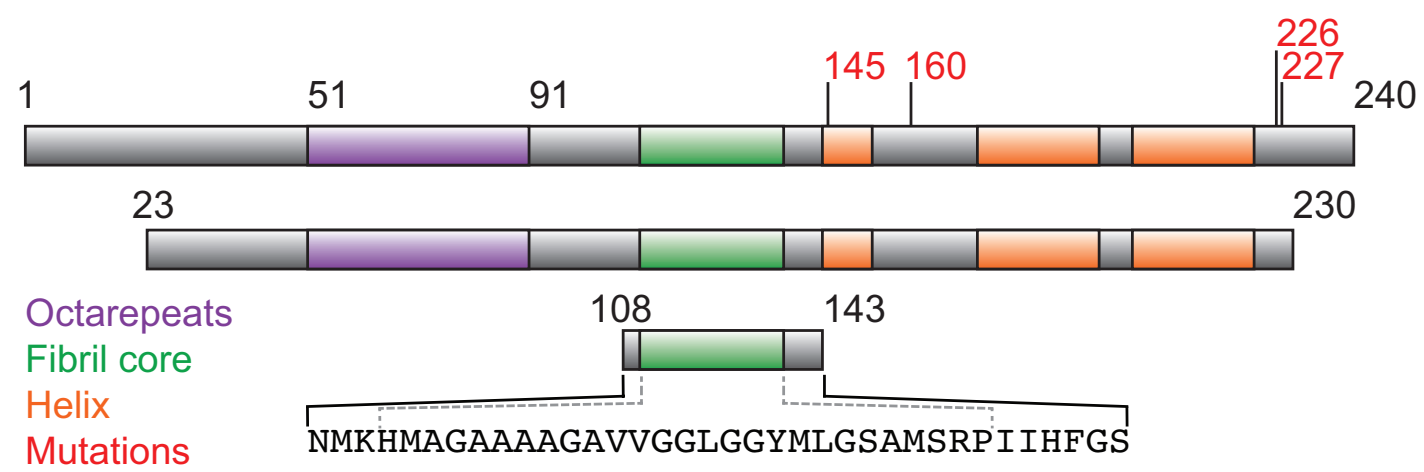

Figure 13. Schematic representation of the main regions and characteristics of the prion protein. The mutation sites in red correspond to inherited forms of prion diseases. Residues 1-22 and 232-240 are cleaved during maturation of the protein. Orange boxes mark the location of a-helices in the native structure of $\operatorname{PrP}$ (reference 3). humPrP(108-143) comprises residues 111-138 (green), which are solventprotected in amyloid fibrils of humPrP(108-143).

We first asked whether histidine 111 and 140 are in the same microenvironment in the free monomeric state. To this end, we performed a $\mathrm{pH}$ titration of the disordered monomeric humPrP(108-143), which probes the response of the two histidine residues to protonation. We changed the $\mathrm{pH}$ from 8 to $\sim 2$ and followed the chemical shift of the two histidines by two-dimensional ${ }^{1} \mathrm{H}-{ }^{1} \mathrm{H}$ TOCSY (TOtal Correlated SpectroscopY). Figure 14 shows that both histidine residues respond in a similar manner as the $\mathrm{pH}$ decreases, resulting in a common $\mathrm{pKa}$ of 5.8. In addition, substitution of histidine 111 by a lysine residue did not affect the $\mathrm{pKa}$ of histidine 140. The analysis showed that the imidiazole ring of both H111 and H140 in the monomeric, unfolded state of humPrP(108-143) has a pKa value close to that observed in an isolated histidine amino acid. 

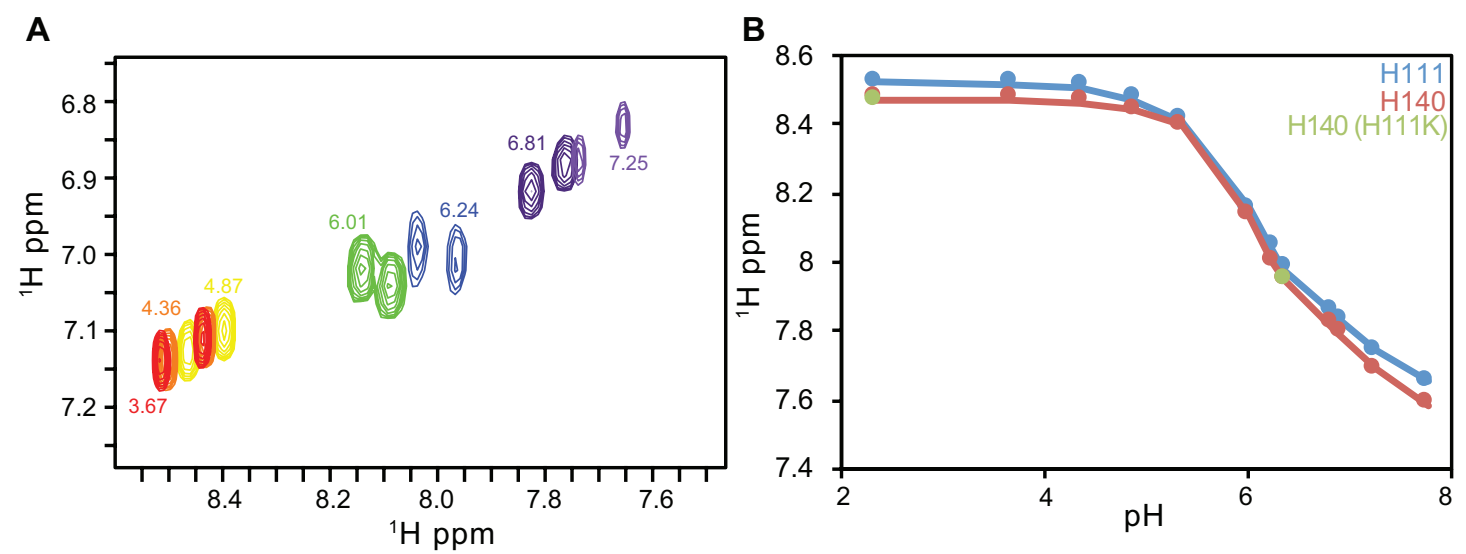

Figure 14. H111 and H140 are exposed to the same microenvironment. (A) Superposition of a selected region from two-dimensional TOCSY spectra of humPrP(108-143) showing cross-peaks of the histidine side-chain protons at increasing $\mathrm{pH}$ (in different colors). $\mathrm{pH}$ values are indicated. (B) Chemical shift change of $\mathrm{H} 111$ and $\mathrm{H} 140$ as a function of $\mathrm{pH}$.

Importance of histidine 111 and 140 for amyloid formation of humPrP(108-143)

Next, we examined the role of histidine 111 and 140 in amyloid formation of humPrP(108-143). To this end, we inserted selected point mutations into the humPrP(108-143) sequence and preformed aggregation assays with both the wildtype and the mutated peptides. Amyloid formation of the peptides was evaluated by binding assays using the amyloid-specific dye thioflavin $\mathrm{T}(\mathrm{ThT})$ as well as electron microscopy (Fig. 15). The measurements showed that replacement of H111 by a positively charged side-chain (lysine) inhibited the ability of humPrP(108-143) to form amyloid fibrils (Fig. 15A). Moreover, introduction of a negatively charged aspartic acid at position 135 , which is in direct spatial proximity to the imidazole ring of H111 in the amyloid fibril model of humPrP(108-143) (S135D, Fig. 15D), restituted its aggregation capabilities (Fig. 15A). The lag phase of amyloid formation 
was only slightly longer in the H111K/S135D variant when compared to the wild-type protein, while its elongation rate was even faster. Consistent with a charge compensation mechanism, a peptide, in which the positive and negative charge at positions 111 and 135 were interchanged, was also able to form amyloid fibrils (Fig. 15C). Notably, the aggregation lag phase of the H111D/S135K variant was roughly half of that of the wild-type peptide, suggesting that additional factors, such as residues in direct proximity of the introduced mutation, might influence the aggregation kinetics of humPrP(108-143). In contrast to these charge effects, substitution of H111 by phenylalanine and S135 by alanine had little effect on the aggregation kinetics of humPrP(108-143) (Fig. 15C, red and green data points, respectively). 
A

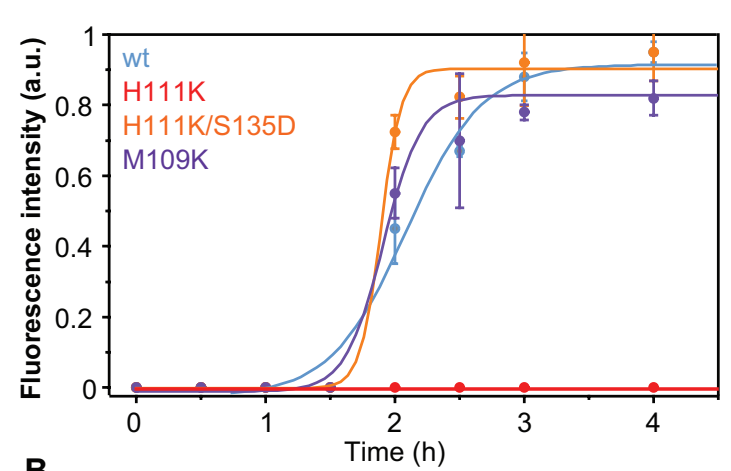

B

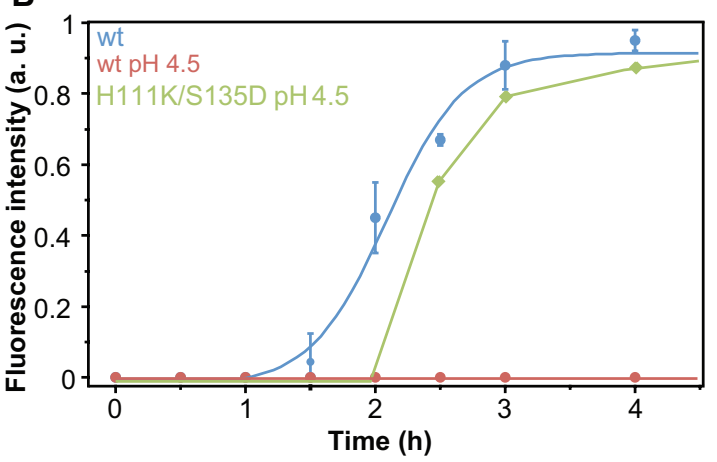

C

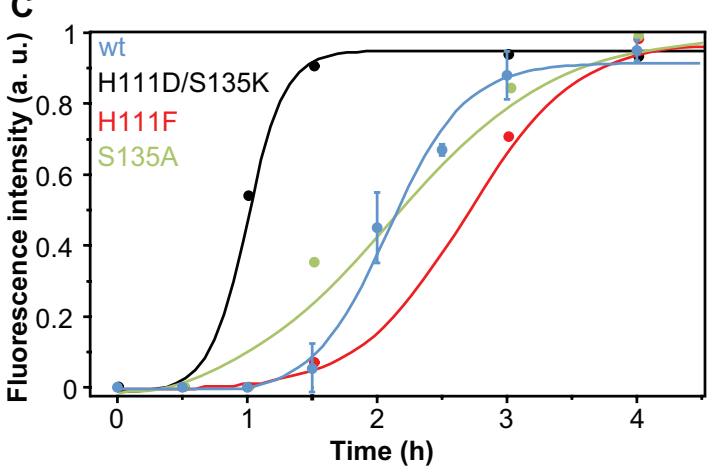

D
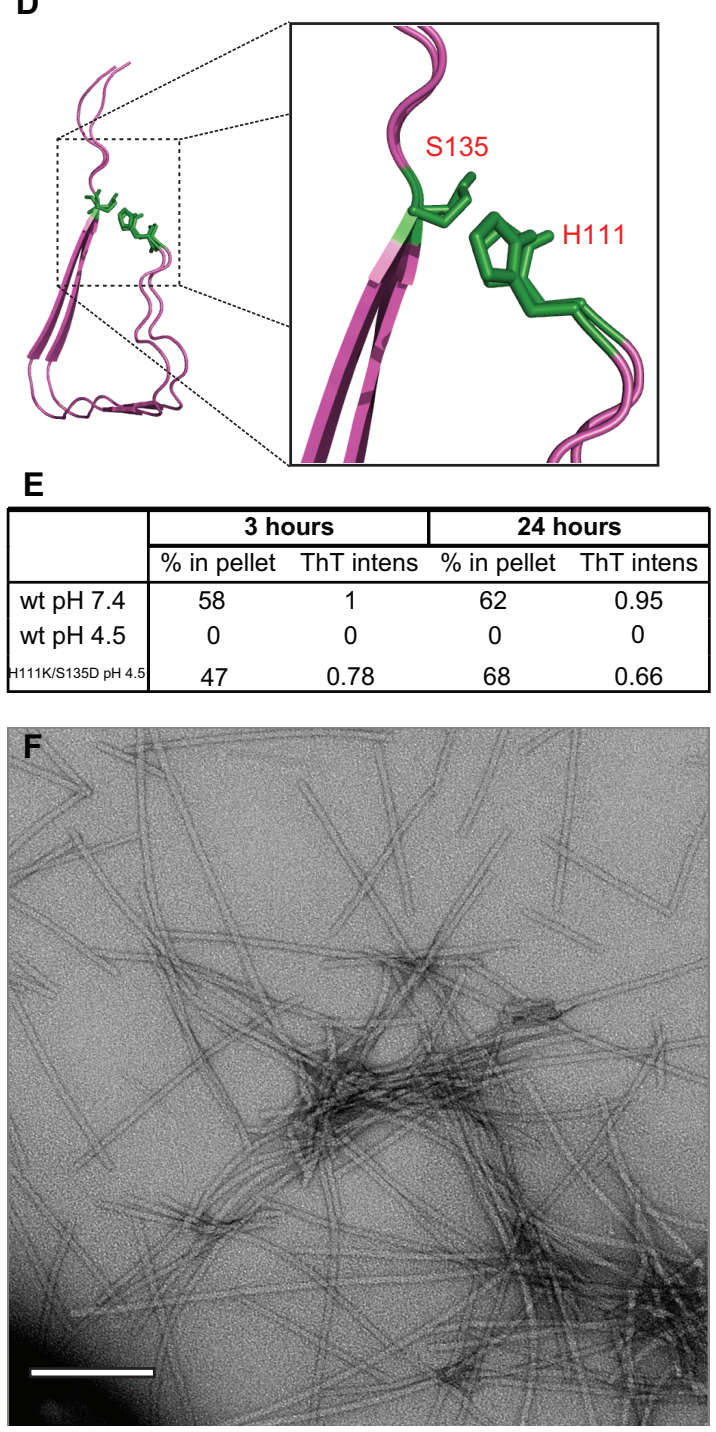

Figure 15. The protonation state of $\mathrm{H} 111$ regulates the aggregation of humPrP(108-143). (A-C) Time-dependent ThT-fluorescence intensity during incubation of different variants of humPrP(108-143). Panel (A) shows that insertion of a positive charge at position $111(\mathrm{H} 111 \mathrm{~K})$ abolishes fibril formation, while neutralizing it with a counteracting negative charge $(\mathrm{H} 11 \mathrm{~K} / \mathrm{S} 135 \mathrm{D})$ restitutes fibrillization. (B) Influence of $\mathrm{pH}$ on aggregation of humPrp(108-143). Protonation of H111 by lowering the $\mathrm{pH}$ to 4.5 prevents fibril formation, while protonation of H140 only slightly extended the lag phase of aggregation (H111K/S135D pH 4.5). (C) Swapping of residues at positions 111 and 135 (H111D/S135K) accelerates aggregation, while insertion of non-charged amino acids (H111F, S135A) only 
slightly changes aggregation kinetics. (E) Estimation of aggregate formation by a protein sedimentation assay. (F) Electron micrograph of amyloid fibrils formed by the $\mathrm{H} 111 \mathrm{~K} / \mathrm{S} 135 \mathrm{D}$ variant of humPrP(108-143) at $\mathrm{pH} 4.5$. The bar represents $200 \mathrm{~nm}$.

The finding that replacement of $\mathrm{H} 111$ by a lysine blocked aggregation of humPrP(108-143) suggested that the protonation state of the imidazole ring of H111 could be an important regulator of the fibrillization of humPrP(108-143). In order to support this hypothesis, we repeated the aggregation of both wild-type humPrP(108143) and the H111K/S135D variant at $\mathrm{pH} 4.5$. Because the NMR-based $\mathrm{pH}$ titration had shown that the pKa value of H111 and H140 is 5.8, the imidazole ring of both histidine residues carries a positive charge at $\mathrm{pH}$ 4.5. All other ionizable residues, on the other hand, should not be strongly affected. Figure $15 \mathrm{~b}$ shows how the decrease to pH 4.5 influenced the aggregation of humPrP(108-143). While wild-type humPrP(108-143) rapidly formed amyloid fibrils at $\mathrm{pH} 7.4$, no fibrillization was detected for it at $\mathrm{pH} 4.5$ by both ThT-fluroescence intensity (Fig. 15B) and protein sedimentation (Fig. 15E). In contrast, H111K/S135D humPrP(108-143) efficiently fibrillized at both $\mathrm{pH} 7.4$ and $\mathrm{pH} 4.5$ (Figs. 15B,E and F). Only the duration of the lag phase was increased by approximately one hour at $\mathrm{pH} 4.5$, while the elongation rate of H111K/S135D humPrP(108-143) was similar at both $\mathrm{pH}$ values. Taken together the data demonstrate that a positive charge in position 111 is able to block the conformational change that is necessary for fibril formation of humPrP(108-143), consistent with its location in the solvent-protected core of the amyloid fibril (Fig. 15D). In contrast, H140 is located outside of the fibril core (Fig. 15D) and does not play a critical role in amyloid formation of humPrP(108-143). 


\section{Influence of sequence homology at position 138/139 for seeding of prion}

\section{aggregation}

Particularly important for the transmission of prion diseases between different species, is the identity of the residues at position 138 and 139 of the prion protein ${ }^{4}$. To investigate the importance of these residues in the context of the $\operatorname{PrP}(108-143)$ sequence, we were prepared two additional mutant peptides. One of these peptides comprised the sequence of the hamster prion protein, which differs from the human one by the substitution of isoleucine 138 and 139 by methionine (Fig. 16A), while in the second variant only I138 was replaced by methionine. The M138/I139 residue combination is found in the sequence of the mouse prion protein. Because the mouse sequence has two additional mutations (M109L and M112V), which were not present, we called the peptide "mod-mouse" (Fig. 16A). Both the hamster and mod-mouse peptides rapidly aggregated in the absence of any aggregation seeds (cyan and pink data points in Fig. A4B), with the hamster peptide having a smaller aggregation lag phase.

Next, we tested if aggregation seeds of either hamster $\operatorname{PrP}(108-143)$ or modmouse $\operatorname{PrP}(108-143)$ are able to seed the aggregation of only the peptide with the identical sequence or also the one, which does not have the same residue types present at positions 138/139. Figure 4B shows that aggregation seeds of both mod-mouse $\operatorname{PrP}(108-143)$ and hamster $\operatorname{PrP}(108-143)$ were able to promote the immediate formation of fibrils when exposed to fresh monomer of both hamster and mod-mouse $\operatorname{PrP}(108-143)$, respectively. The data demonstrate that the difference in sequence in position 139 is not enough to prevent seeding of aggregation. This is consistent with the structural model of amyloid fibrils of humPrP(108-143) (Fig. 15D), because both I138 and I139 are located outside of the structural core, where no tight packing of side 
chains is present. Because previous experiments have shown that mouse and hamster prions do not seed each other ${ }^{5}$, our data suggest that sequence differences between hamster and mouse protein outside of the 138/139 region are more important for the species barrier. This could be in particular residues in the core of the $\beta$-helix shown in Fig. 15D, because this is the most tightly packed region of the amyloid fibrils of humPrP(108-143), in which side chains of different molecules are regularly stacked. To support this hypothesis, we made use of the fibrils, which we obtained from the peptides $\mathrm{H} 111 \mathrm{~F}$ humPrP(108-143) and $\mathrm{S} 135 \mathrm{~A}$ humPrP(108-143), as seeds in the presence of fresh mod-mouse $\operatorname{PrP}(108-143)$ monomer. In this case, the sequence differences are located in the center of the amyloid structure of humPrP(108-143) (highlighted in orange in Fig. 16A). The aggregation profiles were similar to those of the free monomeric peptides, demonstrating that mutations within the amyloid core region block the seeding of aggregation by preformed amyloid fibrils (Fig. 16B).

\section{A}

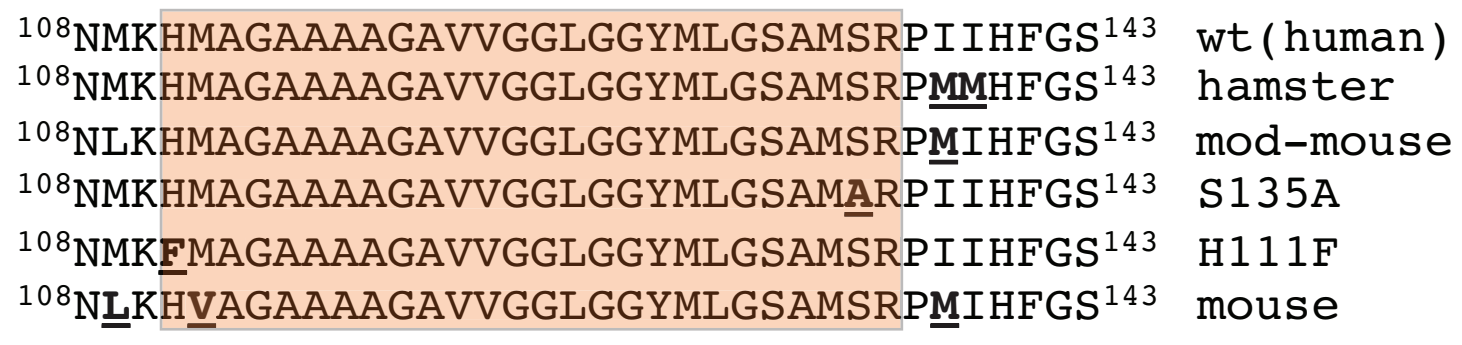

B

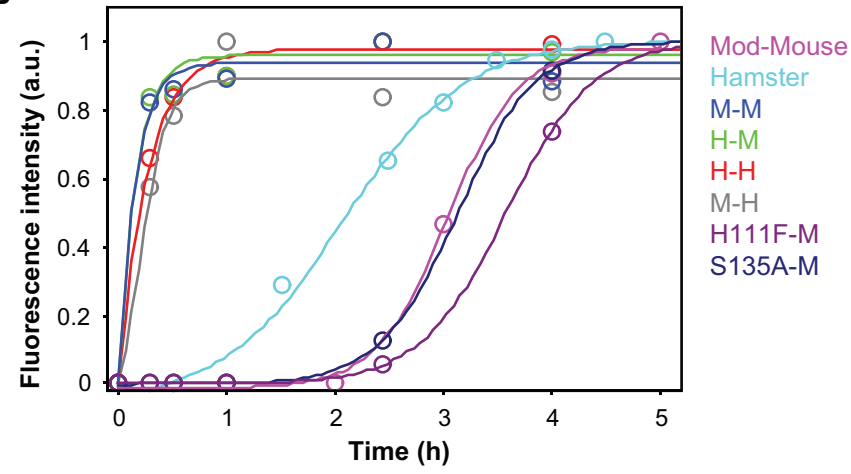

Figure 16. Peptide sequences used for aggregation seeding experiments. (A) 
outside the solvent-protected fibril core (orange region), the seeding potential is not affected. If sequence changes occur within the core, seeding is abolished (B).

\section{Conclusions}

- H111 and H140 share the same microenvironment in the unfolded, monomeric state.

- Residues 111 and 135 are in close proximity in the 3D structure of amyloid fibrils of humPrP(108-143) and their interaction is crucial for fibril formation.

- The protonation state of histidine 111 regulates the aggregation of the highly conserved central region of the prion protein.

- A substantial part of the species barrier is dictated by sequence homology. Especially important for the seeding is sequence identity within the most protected region of the fibril core of humPrP(108-143) comprised by residues 111 to 136.

\section{Appendix references}

1. Skora, L. et al. Burial of the polymorphic residue 129 in amyloid fibrils of prion stop mutants. J. Biol. Chem. (2012). doi:10.1074/jbc.M112.423715

2. Skora, L. \& Zweckstetter, M. Determination of amyloid core structure using chemical shifts. Protein Science 21, 1948-1953 (2012).

3. Zahn, R. et al. NMR solution structure of the human prion protein. Proc. Natl. Acad. Sci. U.S.A. 97, 145-150 (2000).

4. Vanik, D. L., Surewicz, K. A. \& Surewicz, W. K. Molecular basis of barriers for interspecies transmissibility of mammalian prions. Molecular Cell (2004).

5. Lee, L. Y. L. \& Chen, R. P. Y. Quantifying the Sequence-Dependent Species Barrier between Hamster and Mouse Prions. J. Am. Chem. Soc. 129, 1644-1652 (2007). 


\title{
Small molecule mediated stabilization of vesicle-associated helical $\alpha$-synuclein inhibits pathogenic misfolding and aggregation
}

Luis Fonseca-Ornelas ${ }^{1}$, Sybille E. Eisbach ${ }^{2}$, Maria Paulat ${ }^{1}$, Karin Giller ${ }^{1}$, Claudio O. Fernández $^{3}$, Tiago F. Outeiro ${ }^{2,4}$, Stefan Becker ${ }^{1}$ \& Markus Zweckstetter ${ }^{1,4,5}$

${ }^{1}$ Department for NMR-based Structural Biology, Max Planck Institute for Biophysical Chemistry, Am Fassberg 11, 37077 Göttingen, Germany; ${ }^{2}$ Department of Neurodegeneration and Restorative Research, University Medicine, Waldweg 33, 37073, Göttingen, Göttingen, Germany; ${ }^{3}$ Instituto de Biología Molecular y Celular de Rosario, Consejo Nacional de Investigaciones Científicas y Técnicas, Universidad Nacional de Rosario, Suipacha 531, S2002LRK Rosario, Argentina; ${ }^{4}$ DFG Research center for Nanoscale Microscopy and Molecular Physiology of the Brain (CNMPB), University Medical Center, 37073 Göttingen, Germany; ${ }^{5}$ German Center for Neurodegenerative Diseases (DZNE), Am Fassberg 11, 37077 Göttingen, Germany

\begin{abstract}
$\alpha$-synuclein is an abundant presynaptic protein that is important for regulation of synaptic vesicle trafficking, and whose misfolding plays a key role in Parkinson's disease. While $\alpha$-synuclein is disordered in solution, it folds into a helical conformation when bound to synaptic vesicles. Stabilization of helical, folded $\alpha-$ synuclein might therefore interfere with $\alpha$-synuclein induced neurotoxicity. Here we show that several small molecules, which delay aggregation of $\alpha$-synuclein in solution, including the Parkinson's disease drug selegiline, fail to interfere with misfolding of vesicle-bound $\alpha$-synuclein. In contrast, the porphyrin phtalocyanine tetrasulfonate directly binds to vesicle-bound $\alpha$-synuclein, stabilizes its helical conformation and thereby delays pathogenic misfolding and aggregation. Our study suggests that small-molecule mediated stabilization of helical vesicle-bound $\alpha$ synuclein opens new possibilities to target Parkinson's disease and related synucleinopathies.
\end{abstract}




\section{Introduction}

Parkinson's disease is the second most common neurodegenerative disease. It is characterized by specific movement disturbances and affects about $1 \%$ of the population above 60 years ${ }^{1}$. Pathologically, Lewy bodies constitute the hallmark of the disease ${ }^{2}$. Lewy bodies are intraneuronal aggregates formed by misfolded species of the presynaptic protein $\alpha$-synuclein $(\alpha \operatorname{Syn})$, as well as a multitude of proteins and lipids $^{3-7}$. So far, only symptomatic treatment is available for Parkinson's disease and related synucleinopathies. Though the normal function of $\alpha$ Syn is still a matter of debate, recent evidence suggests that $\alpha \mathrm{Syn}$ is important for regulation of synaptic vesicle trafficking and neurotransmitter release via SNARE-complex interaction ${ }^{1,8-10}$.

Under physiological conditions, $\alpha$ Syn is found in equilibrium between a free monomeric species, which samples a large ensemble of dynamic conformations ${ }^{11-13}$, and a membrane-bound $\alpha$-helical structure ${ }^{14-19}$. $\alpha$ Syn preferentially interacts with acidic phospholipids ${ }^{20}$ where it folds into a single continuous helix extending up to approximately residue $100^{21,22}$. In line with the membrane-induced folding of $\alpha$ Syn, two antiparallel, surface-bound helices are formed in the presence of detergent micelles ${ }^{8,23,24}$. When $\alpha$ Syn is bound to vesicles, the highly aggregation-prone NAC (non-amyloid component) region (residues 61-95), is in an $\alpha$-helical conformation and acts as a membrane sensor ${ }^{25}$. Since the NAC region is important for initiating $\alpha$ Syn misfolding and forms the core of amyloid fibrils ${ }^{26}$, stabilization of the helical, vesiclebound conformation of $\alpha$ Syn might interfere with pathogenic aggregation.

Increasing evidence indicates that the most toxic species of $\alpha$ Syn are soluble oligomeric aggregation intermediates ${ }^{10,27-29}$. The search for small chemical compounds that interfere with the aggregation of unfolded, monomeric $\alpha$ Syn into toxic oligomers and fibrils has therefore been intensively pursued using a wide array 
of in vitro and cellular systems as well as animal models of Parkinson's disease $\mathrm{e}^{30-37}$. In contrast, little is known about inhibition of $\alpha$ Syn aggregation in the physiological environment of a vesicle. Indeed, interaction with membranes does not inhibit the potentially pathological process of $\alpha$ Syn aggregation, but can even promote $i^{20,38}$. Moreover, perturbation of the membrane integrity by $\alpha$ Syn, potentially resulting in formation of membrane pores, was suggested to cause neuronal death ${ }^{39}$.

Here, we demonstrate that several small molecules that were previously shown to delay aggregation of soluble, disordered $\alpha \mathrm{Syn}$, are not able to inhibit aggregation of vesicle-bound $\alpha$ Syn. In contrast, the polyphenol phtalocyanine tetrasulfonate (PcTS) directly binds to vesicle-bound $\alpha$ Syn, stabilizes its helical conformation and inhibits misfolding and aggregation. We further show that PcTS prevents inclusion formation and toxicity in a cell model of $\alpha$ Syn aggregation. Thus, our study opens novel possibilities for interfering with aSyn misfolding and neurotoxicity in its physiological environment.

\section{Results}

PcTS stabilizes the helical structure of lipid-bound $\alpha$ Syn. A multitude of organic compounds from different chemical classes were shown to modulate aggregation of aSyn in solution ${ }^{30-33,40}$. This includes the polyphenols curcumin, baicalein and epigallocatechin gallate (EGCG), the drug selegiline, which is used for treatment of early-stage Parkinson's disease, and the porphyrin PcTS (Fig. 1a). In agreement with previous studies, curcumin, baicalein, and EGCG inhibited almost completely the formation of $\alpha$ Syn fibrils in solution when used at a 15:1 compound-to-protein ratio (Supplementary Fig. 1). Selegiline and PcTS reduced ThT staining to a smaller degree (Supplementary Fig. 1). PcTS was previously shown to favor prefibrillar $\alpha$ Syn species $^{34}$. 

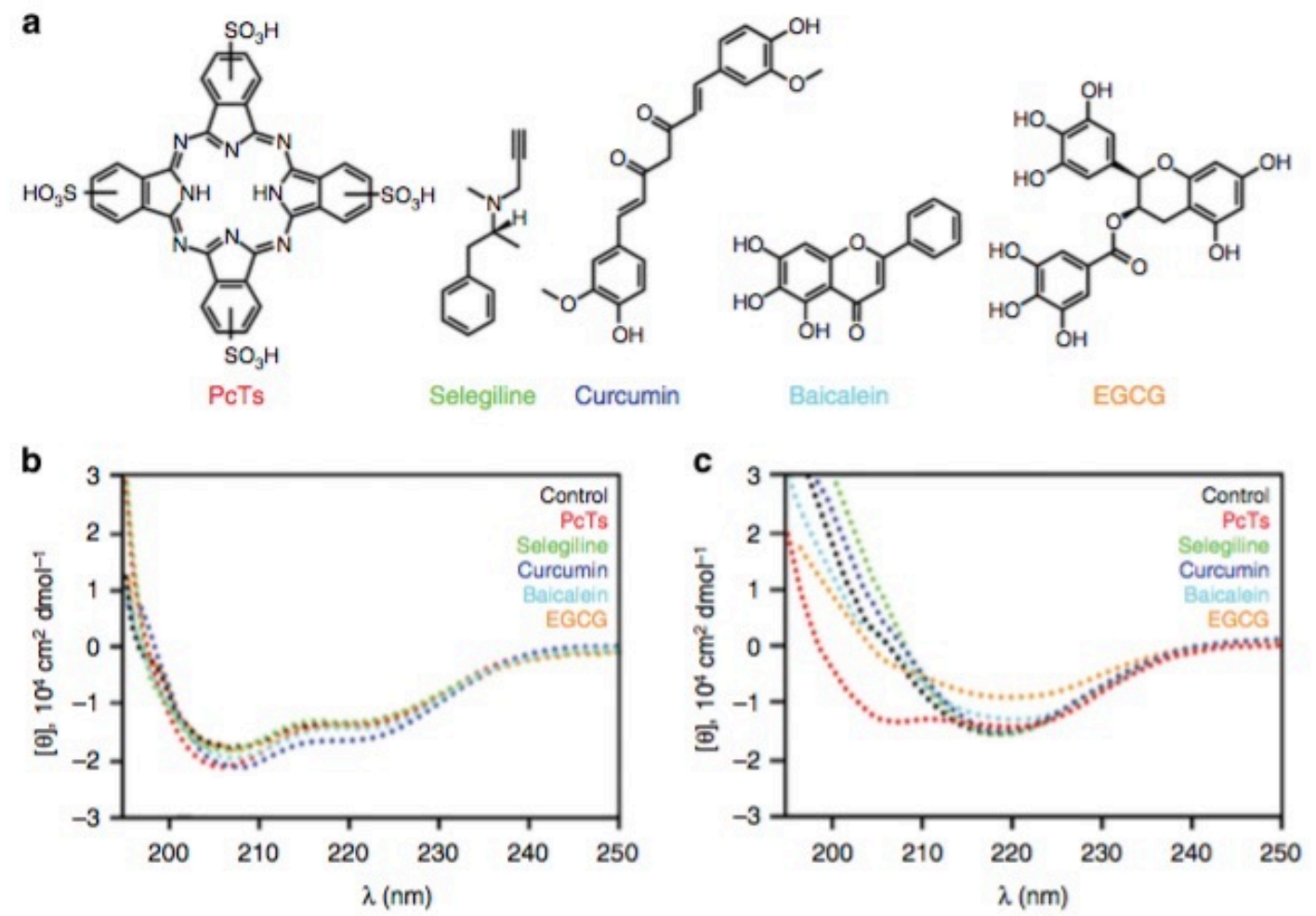

d
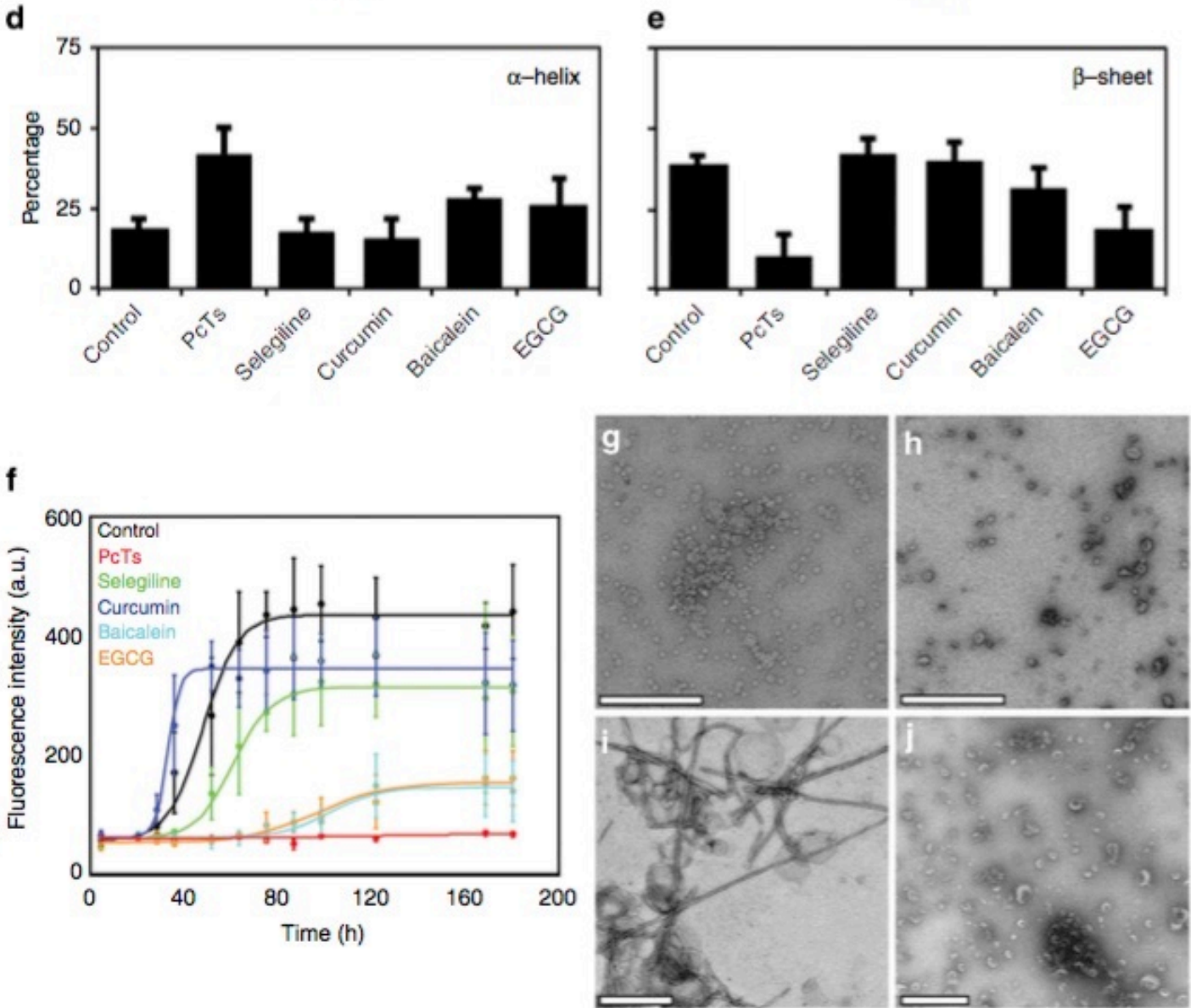

Figure 1 | A small organic molecule stabilizes helical, vesicle-bound $\alpha$ Syn and inhibits its aggregation. (a) Chemical structure of PcTS, selegiline, curcumin, 
baicalein and EGCG. (b,c) Circular dichroism spectra of $\alpha$ Syn before (b) and after incubation for 10 days in aggregation-prone conditions (c), both in the absence and presence of a 15:1 excess of PcTS (red), selegiline (green), curcumin (blue), baicalein (light blue) and EGCG (orange). The lipid-to-protein molar ratio was 125:1. In the presence of PcTS, the $\alpha$-helix content was $\sim 41 \%$ and less than $10 \%$ of $\beta$-structure was formed after ten days of incubation in aggregation-prone conditions. (d-f) $\alpha$-helix (d) and $\beta$-sheet (e) content together with ThT fluorescence (f) aggregation kinetics of vesicle-bound aSyn. The control did not contain any small molecule. Error bars represent \pm standard deviation from the mean of at least 3 different experiments. As the C-terminal domain remains disordered in vesicle-bound $\alpha \operatorname{Syn}^{13}$, the maximum $\alpha$ helical content is $\sim 70 \%$. In $w t$ SSyn fibrils, the central domain (approximately residues 38-96 with some loops) is converted into $\beta$-structure ${ }^{60}$, corresponding to a $\beta$ structure content of $\sim 40 \%$. (g-j) Electron micrographs of fresh (g,h) and aggregated (i,j) $\alpha$ Syn/SUV samples: control (g,i) and PcTS (h,j). The white bar represents 500 $\mathrm{nm}$.

Next we tested if curcumin, selegiline, baicalein, PcTS and EGCG interfere with the binding and folding of aSyn on the surface of small unilamellar vesicles $\left(\mathrm{SUVs}^{21}\right)^{21}$ To address this question, we formed SUVs from a 1:1 mixture of 1palmitoyl-2-oleoyl phosphatidylcholine (POPC) and 1-palmitoyl-2-oleoyl phosphatidic acid (POPA). NMR spectroscopy showed that selegiline, baicalein and EGCG interact with POPA/POPC liposomes (Supplementary Fig. 2). Nevertheless, none of the compounds impaired vesicle-binding of $\alpha$ Syn and its folding into an $\alpha$ helical conformation even at a 15 -fold excess of compound with respect to $\alpha$ Syn (Fig. $1 \mathrm{~b}$ and Supplementary Fig. 3). 
Are the small molecules able to modulate the aggregation of $\alpha$ Syn in the presence of vesicles? To address this question, $\alpha$ Syn was subjected to aggregationpromoting conditions $\left(37^{\circ} \mathrm{C}\right.$, constant stirring) for ten days in the absence and presence of the small molecules. When no compound was present, the $\alpha$-helical content of $\alpha$ Syn decreased from $\sim 70 \%$ to below $25 \%$ (Fig. 1b,c,d). In parallel, $\beta$ structure was formed, the sample stained for the amlyoid-dye thioflavin $\mathrm{T}(\mathrm{ThT})$ and electron microscopy revealed fibrillar structures (Fig. 1c,e,f,i), consistent with previous results ${ }^{1,34,41}$. Despite its strong anti-aggregation capacity toward soluble $\alpha$ Syn (Supplementary Fig. 1), curcumin did not block the transition from $\alpha$-helix to $\beta$ structure of vesicle-bound $\alpha$ Syn nor the formation of amyloid fibrils (Fig. 1c,d,e,f and Supplementary Fig. 4). Selegiline, baicalein and EGCG also did not interfere with the loss of $\alpha$-helical structure (Fig. 1c,d), although samples aggregated in the presence of EGCG and baicalein stained only weakly for ThT, probably as a result of competition with the compounds for binding sites on $\alpha$ Syn fibrils (Fig. 1f). The amount of fibrils observed in EM micrographs in the presence of EGCG was small (Supplementary Fig. 4), indicating that EGCG directs $\alpha$ Syn into off-pathway aggregates both in solution $^{32}$ and in the presence of vesicles. Curcumin, baicalein, selegiline and EGCG were used at a 15:1 compound-to-protein ratio. The reported apparent binding constants of curcumin and baicalein to $\alpha$ Syn are $10 \mu \mathrm{M}$ and $500 \mathrm{nM}$, respectively, while selegiline and EGCG do not bind to monomeric $\alpha$ Syn, but interact with oligomeric species $^{30,31,33,42,43}$.

In contrast to curcumin, selegiline, baicalein and EGCG, no ThT signal was detected after ten days of incubation of vesicle-bound $\alpha$ Syn when PcTS was present (Fig. 1f). In addition, EM micrographs were free of fibrillar structures and the vesicle morphology appeared unmodified (Fig. 1h,j). Quantification of the secondary 
structure indicated that even after 10 days of incubation in aggregation-prone conditions the $\alpha$-helix content remained at $\sim 41 \%$ and less than $10 \%$ of $\beta$-structure was formed (Fig. 1c,d,e). Thus, PcTS is able to stabilize the helical, membrane-bound conformation of $\alpha$ Syn and inhibit its aggregation in the presence of vesicles. PcTS also inhibited $\alpha$ Syn aggregation in the presence of large unilamellar vesicles (LUVs) (Fig. 2). Experiments at different $\alpha$ Syn:compound ratios further showed that a 5:1 but not a 1:1 PcTS: $\alpha$ Syn ratio was sufficient to inhibit amyloid formation (Supplementary Fig. 5).

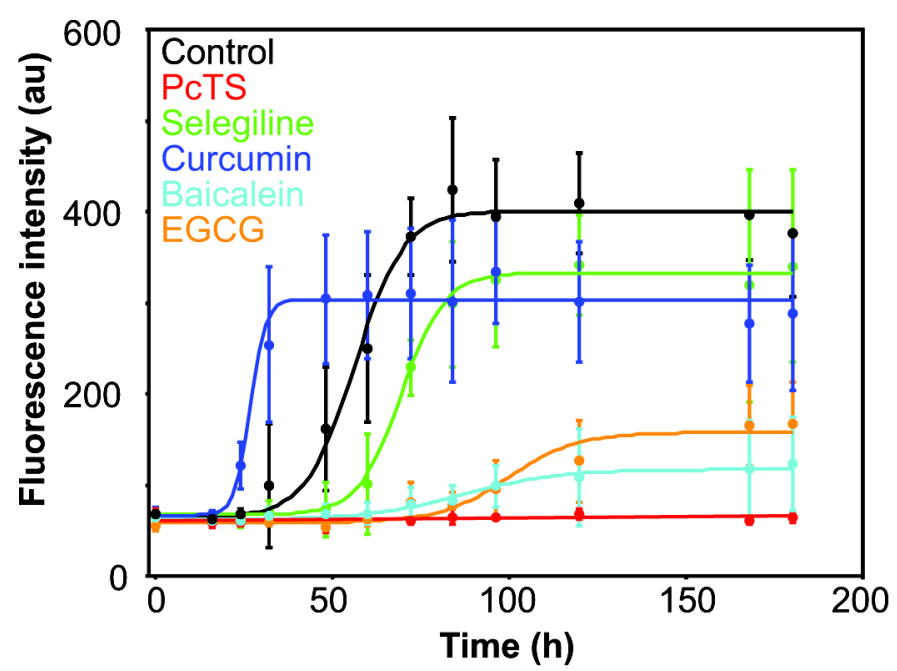

Figure 2 | Influence of selected small molecules on fibril formation of $\alpha \mathrm{Syn}$ in the presence of a $\sim 12.5 \mathrm{mM}$ concentration of large unilamellar vesicles (LUVs). Fibril formation kinetics in aggregation prone conditions $\left(37{ }^{\circ} \mathrm{C}\right.$ with stirring). The concentration of $\alpha$ Syn was $100 \mathrm{mM}$. 15-fold excess of compound over protein was used. The final DMSO concentration was $1 \%$ of the total volume. Average values of at least 3 different samples. Error bars represent \pm standard deviation from the mean of at least 3 different experiments.

PcTS releases the NAC region from the vesicle surface. To obtain insight into the mechanism of the stabilization of the $\alpha$-helical structure of membrane-bound $\alpha$ Syn by PcTS we used solution NMR spectroscopy. Although the slow tumbling rate of 
vesicles induces lipid-bound $\alpha$ Syn to exhibit dynamic properties that hamper its direct observation by solution NMR, exchange with the NMR-visible, lipid-free form allows residue-specific access to the properties of the membrane-bound state ${ }^{24}$. We probed this exchange process using two-dimensional ${ }^{15} \mathrm{~N}-{ }^{1} \mathrm{H}$ heteronuclear single quantum coherence (HSQC) experiments of $100 \mu \mathrm{M} \alpha \mathrm{Syn}$ in the presence of $12.5 \mathrm{mM}$ SUVs (1:125 protein-to-lipid ratio). In line with previous studies ${ }^{24}$, the NMR signal intensities of the $100 \mathrm{~N}$-terminal residues were strongly decreased compared to free $\alpha$ Syn (Fig. 3a red spectrum; 3b, red line). The observed signal originates from unbound $\alpha$ Syn, as the bound protein does not contribute ${ }^{24}$. In the C-terminal region, the NMR resonances had a comparable intensity as in the free state, indicating that they interact at best weakly with vesicles.
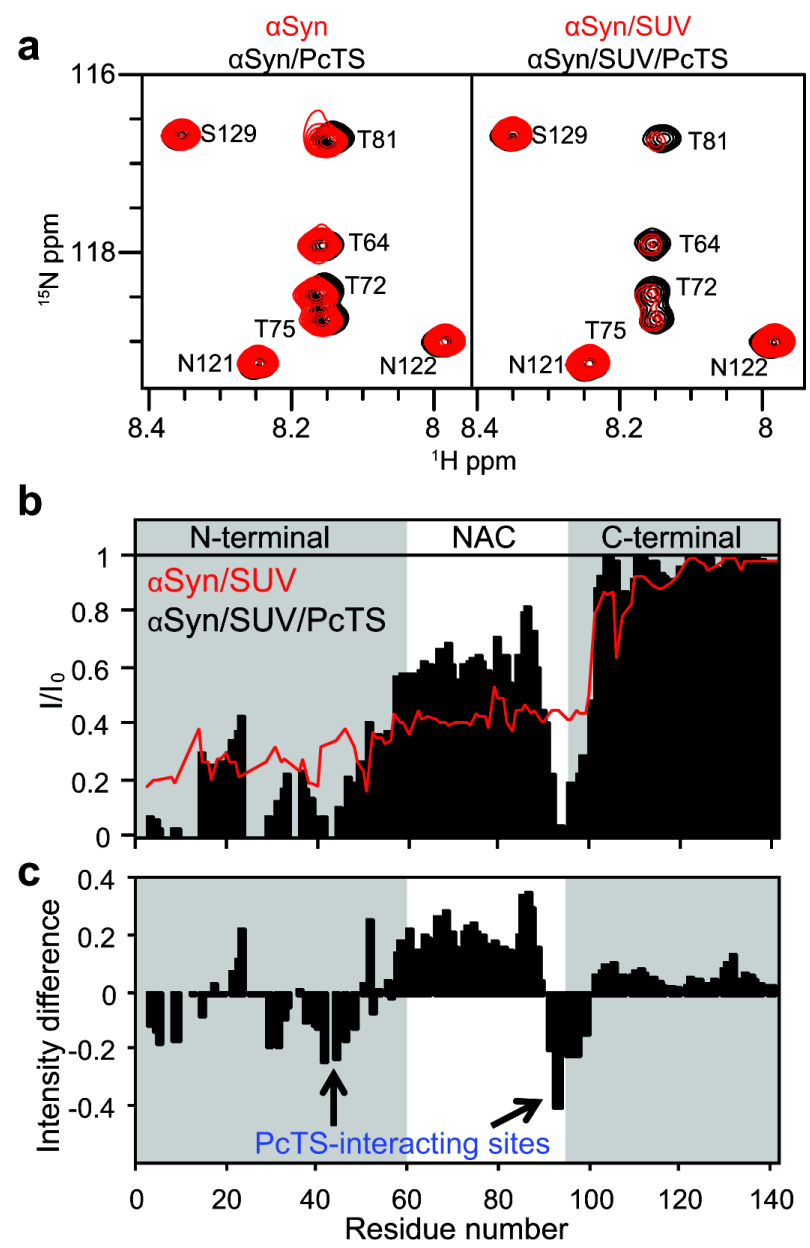
Figure 3 (previous page) | PcTS binding partially releases $\alpha$ Syn's NAC region from vesicles. (a) Selected region of $2 \mathrm{D}{ }^{15} \mathrm{~N}-{ }^{1} \mathrm{H}$ HSQC spectra of free (left) and vesicle-bound $\alpha$ Syn (right) in the absence (red) and presence of a 15-fold excess of PcTS (black). NMR signals of residues in the NAC region are significantly broadened when in contact to liposomes (right panel). Upon PcTS addition (black spectrum), cross-peaks of these amino acids gain in intensity, indicating a partial release from the vesicle surface. (b) Quantification of NMR signal intensities along the aSyn sequence when bound to SUVs in the absence (red line) and the presence of a 15-fold excess of PcTS (black bars). Signal intensities $(I)$ were normalized by the values observed for $\alpha$ Syn in the absence of SUVs $\left(I_{0}\right)$. The lipid-to-protein molar ratio was $125: 1$. White color marks the aggregation-prone NAC region (residues 61-95). Note that when residues are no longer in contact with liposomes, they become "visible" to solution NMR. (c) Quantification of the signal intensity difference upon addition of PcTS. The intensity decrease around Y39 and F94 is due to the binding of PcTS in free $\alpha$ Syn. 

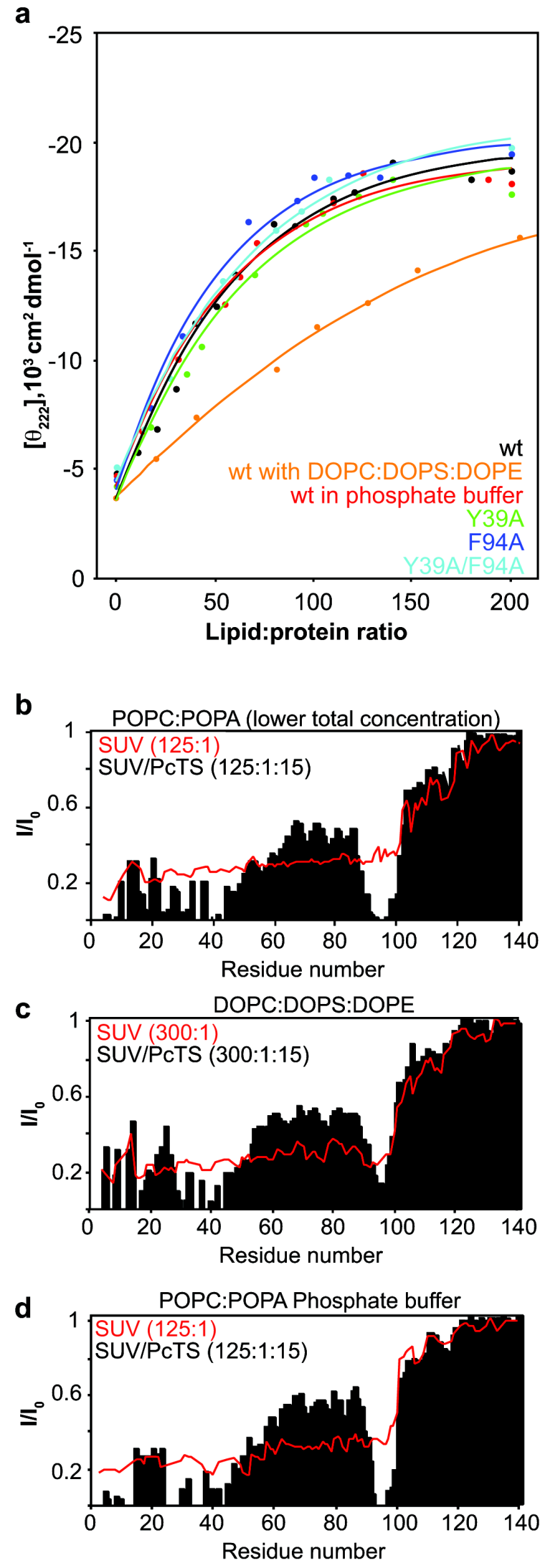

Figure 4 | Influence of mutations, buffer composition and lipid composition on binding of $\alpha$ Syn to vesicles. (a) Mean residue ellipticity of $\alpha$ Syn variants for 
increasing lipid-to-protein ratios. When not indicated otherwise SUVs were composed of a 1:1 mixture of POPA:POPC and the buffer system was $5 \mathrm{mM}$ HEPES buffer, 10 $\mathrm{mM} \mathrm{NaCl}, \mathrm{pH}$ 7.4. In addition, data for the wild-type protein in $5 \mathrm{mM}$ sodium phosphate buffer, $10 \mathrm{mM} \mathrm{NaCl}$ (red) are shown. The Y39A, F94A and Y39A/F94A mutations do not attenuate $\alpha$ Syn binding to liposomes (a; green, blue and cyan, respectively). (b-d) Quantification of NMR signal intensities along the $\alpha$ Syn sequence when bound to SUVs in the absence (red line) and the presence of a 15-fold excess of PcTS (black bars). Signal intensities $(I)$ were normalized by the values observed for aSyn in the absence of SUVs $\left(I_{0}\right)$. (b) When compared to Fig. 3b, the same molar ratios of $\alpha$ Syn:SUVs:PcTS were used, but at an overall 5-fold decreased concentration (2.5 mM SUVs, $20 \mu \mathrm{M} \alpha \mathrm{Syn}, 300 \mu \mathrm{M}$ PcTS). The lipid-to-protein molar ratio was 125:1. (c) NMR signal broadening profile in the presence of vesicles composed of DOPE, DOPS and DOPC at a molar ratio of 5:3:2. Because of the lower affinity of aSyn to DOPE:DOPS:DOPC vesicles (when compared to POPA:POPC vesicles) a higher lipid-to-protein ratio was used. (d) Effect of PcTS on the liposome-induced NMR signal broadening profile of $\alpha$ Syn in a $50 \mathrm{mM}$ sodium phosphate buffer containing $10 \mathrm{mM} \mathrm{NaCl}, \mathrm{pH}$ 7.4. SUVs were composed of a 1:1 mixture of POPA:POPC. NMR measurements were performed at $15^{\circ} \mathrm{C}$.

Upon addition of $1.5 \mathrm{mM}$ PcTS (15:1 compound-to-protein ratio), the crosspeaks of residues in proximity to Y39 and F94 as well as at the N-terminus were further attenuated and shifted (Fig. 3a,b,c and Supplementary Fig. 6). The intensity decrease and chemical shift changes are caused by the binding process and were previously observed upon addition of PcTS to soluble $\alpha \mathrm{Syn}^{34}$. It shows that PcTS is in solution and can bind to the residual unbound protein. In contrast, residues within the 
NAC region (residues 61-95) gained approximately 20 to 30\% of intensity (Fig. 3a, black spectrum; Fig. 3b, black bars; Fig. 3c). In addition, at higher lipid-to-protein ratios, when no NMR signals are observed for the N-terminal 100 residues, addition of PcTS led to the appearance of resonances from the NAC region (Supplementary Fig. 7). Thus, PcTS promotes a faster exchange of the NAC region between the vesicle-bound and free state, making NAC residues "visible" in the NMR spectrum. Using a different buffer system (50 mM phosphate, $\mathrm{pH} 7.4)$ and/or different liposome composition (DOPE, DOPS and DOPC in a 5:3:2 ratio) yields the same behavior in which the NAC region of $\alpha$ Syn is released from its interaction with the membrane (Fig. 4). Also, the concentrations of PcTS used here are not sufficiently large to interfere with the formation of liposomes (Supplementary Fig. 8). In contrast to PcTS, addition of curcumin, selegiline, and baicalein did not change the NMR signal intensity profile of $\alpha \mathrm{Syn}$ in the presence of POPC/POPA vesicles (Fig. 5). EGCG further reduced the NMR signal intensity of residues 1-130, consistent with the EGCG-mediated conversion of $\alpha$ Syn molecules, which are not bound to the vesicles, into off-pathway oligomeric species.

Interaction with Y39 and F94 drives NAC-domain release. It was previously shown that PcTS directly binds to monomeric, soluble $\alpha \mathrm{Syn}^{34}$. The interaction is driven by aromatic stacking with F4, Y39 and F94. In order to study the importance of Y39 and F94 for binding of PcTS to membrane-bound $\alpha$ Syn, we generated the aSyn mutants Y39A and F94A, as well as the double mutant Y39A/F94A. The SUVinduced intensity profiles of the mutants were similar to that of wild-type $(w t) \alpha$ Syn (Fig. 6a-c), indicating a similar affinity and mechanism of membrane binding. This was confirmed by determination of a dissociation constant of $\sim 60 \pm 15 \mu \mathrm{M}$ in all cases 
(assuming a one-site binding model; Fig. 5a). It also suggests that Y39 and F94 are not important for anchoring $\alpha$ Syn to POPA/POPC vesicles, consistent with the observation that Y39 is primarily water exposed when $\alpha$ Syn is bound to vesicles ${ }^{44}$.

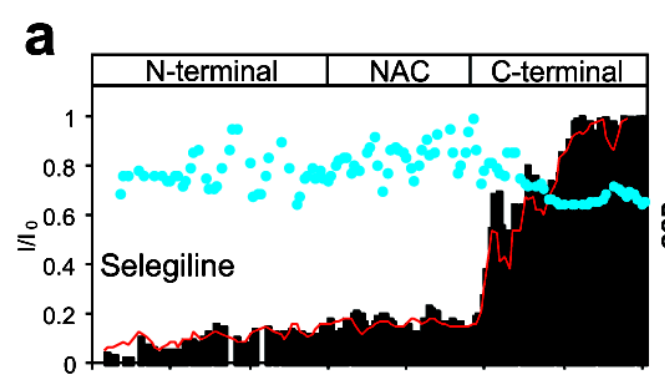

b
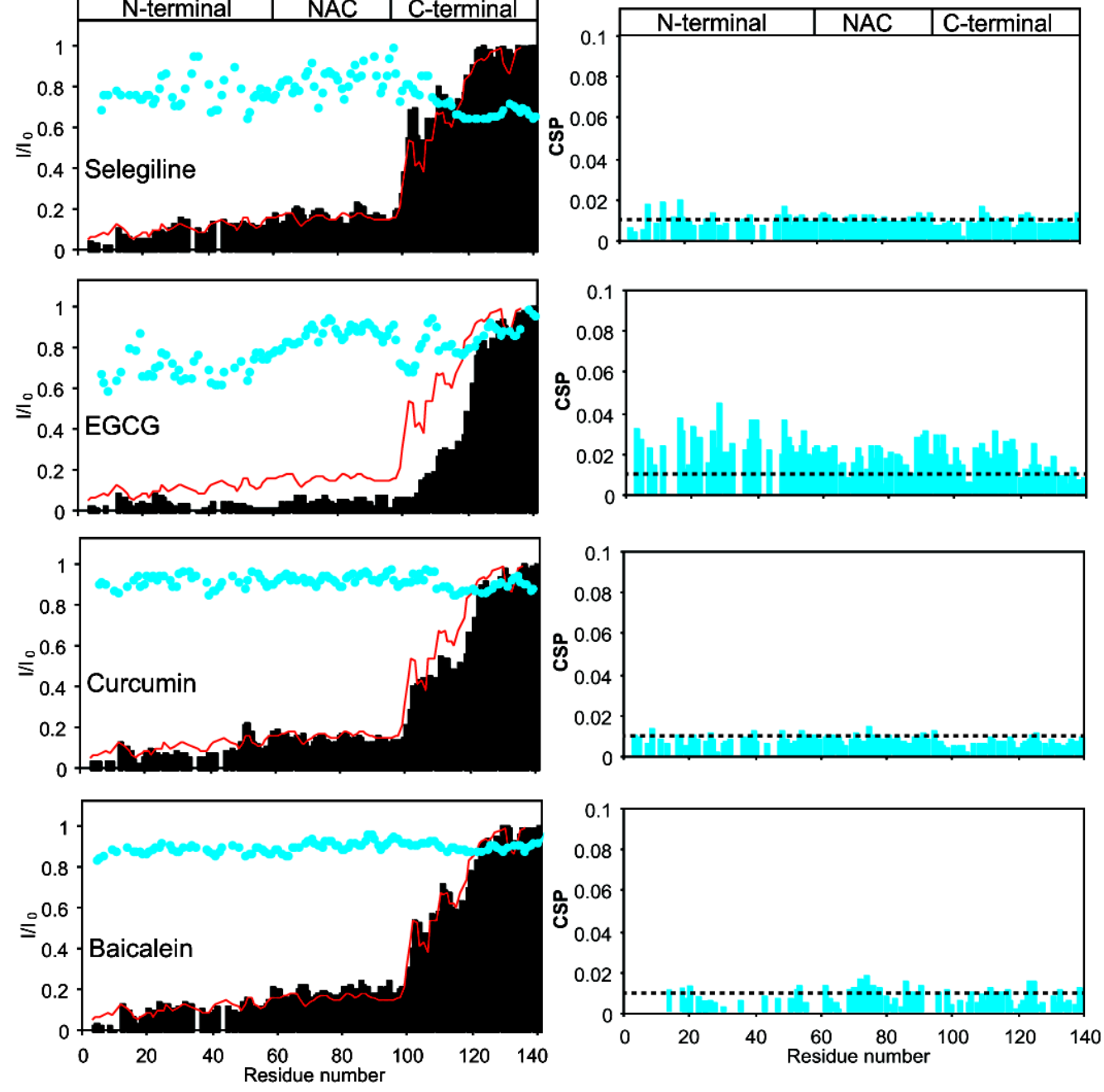

Figure 5 | Interaction of selegiline, EGCG, curcumin and baicalein with soluble and vesicle-bound aSyn. (a) Quantification of NMR signal intensities along the $\alpha$ Syn sequence when bound to SUVs in the absence (red line) and the presence of a 15-fold excess of selegiline, EGCG, curcumin and baicalein (black bars). Signal intensities ( $I$ ) were normalized by the values observed for $\alpha$ Syn in the absence of SUVs $\left(I_{0}\right)$. The lipid-to-protein molar ratio was 125:1. The ratio of NMR signal intensities of free (in the absence of SUVs) $\alpha$ Syn in the presence and absence of the 
respective compound are shown in light blue. (b) Chemical shift differences for free $\alpha$ Syn in the absence and presence of the compounds. EGCG causes intensity and chemical shift changes due to its binding to monomeric $\alpha$ Syn and conversion of $\alpha$ Syn into off-pathway oligomers. The dashed lines represent the intrinsic error for the chemical shift measurement.

We then added PcTS to the $\alpha$ Syn variants in complex with vesicles. In the presence of a 15-fold excess of PcTS, NMR signal intensities in the NAC region of the Y39A mutant increased by about $15 \%$ (Fig. 6a), while the wt protein regained about $20-30 \%$ (Fig. 3b,c). In case of the F94A variant of $\alpha$ Syn, the PcTS-induced signal increase remained also below that of $w t \alpha$ Syn and was only slightly larger than for the Y39A mutant (Fig. 6b). Notably, simultaneous mutation of Y39 and F94 abolished the PcTS-induced signal increase in the NAC region (Fig. 6c). Thus, the partial release of $\alpha$ Syn's NAC residues from the membrane-surface is specifically due to the interaction of PcTS with Y39 and F94.

To obtain further insight into the aSyn-membrane interaction, we performed ${ }^{15} \mathrm{~N}$ spin relaxation measurements. Previous experiments had shown that the exchange with the membrane-bound $\alpha$-helical state enhances the relaxation of the NMR resonances of disordered soluble $\alpha \operatorname{Syn}^{24,45}$. Consistent with this finding, addition of SUVs increased the transverse ${ }^{15} \mathrm{~N}$ spin relaxation rate from $\sim 3 \mathrm{~s}^{-1}$ to $\sim 14 \mathrm{~s}^{-1}$ for residues 1-40 and to $\sim 12 \mathrm{~s}^{-1}$ for residues 40-100 (Fig. 6d). In the presence of PcTS, relaxation rates in the $\mathrm{N}$-terminal part of $w t$ aSyn remained high, while in the NAC region the average ${ }^{15} \mathrm{~N}$ transverse relaxation rate decreased from $\sim 12 \mathrm{~s}^{-1}$ to $\sim 7 \mathrm{~s}^{-1}$ (Fig. $6 \mathrm{~d}, \mathrm{e})$. The decreased contribution of the vesicle-bound state to the observed spin relaxation rate supports a PcTS-induced partial release of the NAC region of $w t \alpha \mathrm{Syn}$ 
from the vesicle surface. In contrast, no PcTS-induced changes in relaxation rates were observed for the Y39A/F94A variant of $\alpha$ Syn (Fig. 6e,f), in agreement with the importance of Y39 and F94 for PcTS-binding and NAC domain release.
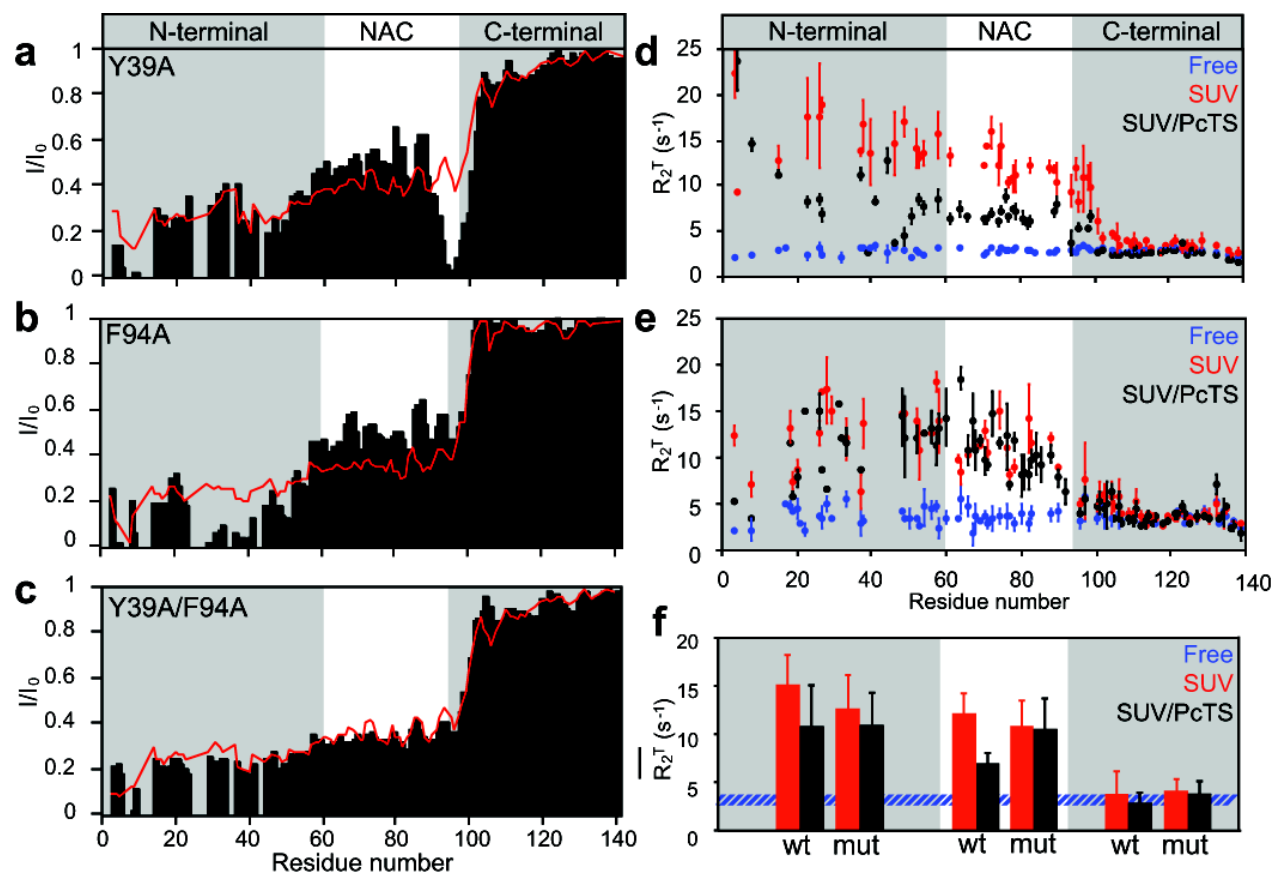

Figure 6 | Y39 and F94 are essential for PcTS-induced membrane detachment of aSyn. a-c Residue-specific NMR signal intensities in the $\alpha$ Syn variants (a) Y39A, (b) F94A and (c) Y39A/F94A in the presence of SUVs without (red line) and with a 15fold excess (black bars) of PcTS. Signal intensities $(I)$ were normalized by the values observed for each $\alpha$ Syn variant in the absence of SUVs $\left(I_{0}\right)$. The lipid-to-protein molar ratio was $125: 1$. White color marks the aggregation-prone NAC region. In the absence of the two aromatic residues Y39 and F94, PcTS does not affect the binding of aSyn to vesicles. (d) ${ }^{15} \mathrm{~N} \mathrm{R}_{2}{ }^{\mathrm{T}}$ transverse spin-relaxation rates in $w t \alpha \mathrm{Syn}$ in the absence of vesicles (blue), the presence of SUVs (red) and after further addition of a 15-fold excess of PcTS (black). The lipid-to-protein molar ratio was 6:1. In the presence of SUVs, the relaxation rates increase at the N-terminus and the NAC region due to the exchange with the vesicle-bound state (red). PcTS-induced release of the NAC region increases the exchange between free and vesicle-bound $\alpha$ Syn causing a 
decrease in $\mathrm{R}_{2}{ }^{\mathrm{T}}$ values (black). (e) ${ }^{15} \mathrm{~N} \mathrm{R}_{2}{ }^{\mathrm{T}}$ rates in the Y39A/F94A $\alpha$ Syn variant. Due to the depletion of the binding sites, PcTS does not influence the exchange of this $\alpha$ Syn variant with membranes. As in (d), error bars represent curve fitting errors. (f) $\mathrm{R}_{2}{ }^{\mathrm{T}}$ values averaged over residues 1-60 (grey), 61-95 (white) and 96-140 (grey) in both $w t$ and Y39A/F94A (mut) $\alpha$ Syn. Values in the absence and presence of PcTS are shown by red and black bars. The average value of $\mathrm{R}_{2}{ }^{\mathrm{T}}$ in free $w t \alpha \mathrm{Syn}$ is shown as blue horizontal line. Error bars indicate standard deviation.

A distinct aggregation pathway of vesicle-bound $\boldsymbol{\alpha S y n}$. Next we asked if the PcTSmediated inhibition of aggregation of vesicle-bound $\alpha$ Syn (Fig. 1) is connected to the PcTS-induced partial release of $\alpha$ Syn's NAC region from the membrane surface (Figs. 3,6). To address this question, we analyzed the influence of PcTS on the aggregation of both $w t$ and mutant $\alpha$ Syn in the presence of vesicles (Fig. 7). While PcTS completely blocked aggregation of vesicle-bound wt $\alpha$ Syn, the $\alpha$ Syn variants were able to form amyloid fibrils in the presence of PcTS (Figs. 1 and 7). Aggregation of the Y39A/F94A double mutant in the presence of PcTS even approached the aggregation behavior of $w t \alpha$ Syn in the absence of PcTS (Fig. 7), despite the fact that its vesicle-induced broadening profile did not differ from that of wt aSyn (Fig. 6c). The slower fibrillization rate of the single point mutants when compared to Y39A/F94A $\alpha$ Syn (Fig. 7a) is in agreement with a PcTS-induced partial release of the NAC region of Y39A $\alpha$ Syn and F94A $\alpha$ Syn from the vesicle surface (Fig. 6a,b). The data demonstrate that membrane-attachment of the NAC region is essential for aggregation of $\alpha$ Syn in a lipid environment.

It was previously shown that Y39 is important for aSyn aggregation in solution. When Y39 was mutated to alanine, the formation of fibrils was strongly 
delayed (Fig. 8) ${ }^{34}$. Given the differences at the micro-environmental level between free and vesicle-bound aSyn, we asked if the presence of this aromatic residue at position 39 plays a similar role for vesicle-bound $\alpha$ Syn. However, the Y39A mutation did not interfere with the aggregation of vesicle-bound $\alpha$ Syn (Fig. 8). The distinct aggregation behavior of the Y39A variant of $\alpha$ Syn in solution and when bound to vesicles demonstrates that the early stages of aggregation differ drastically between the free, disordered and the membrane-bound $\alpha$-helical $\alpha$ Syn.
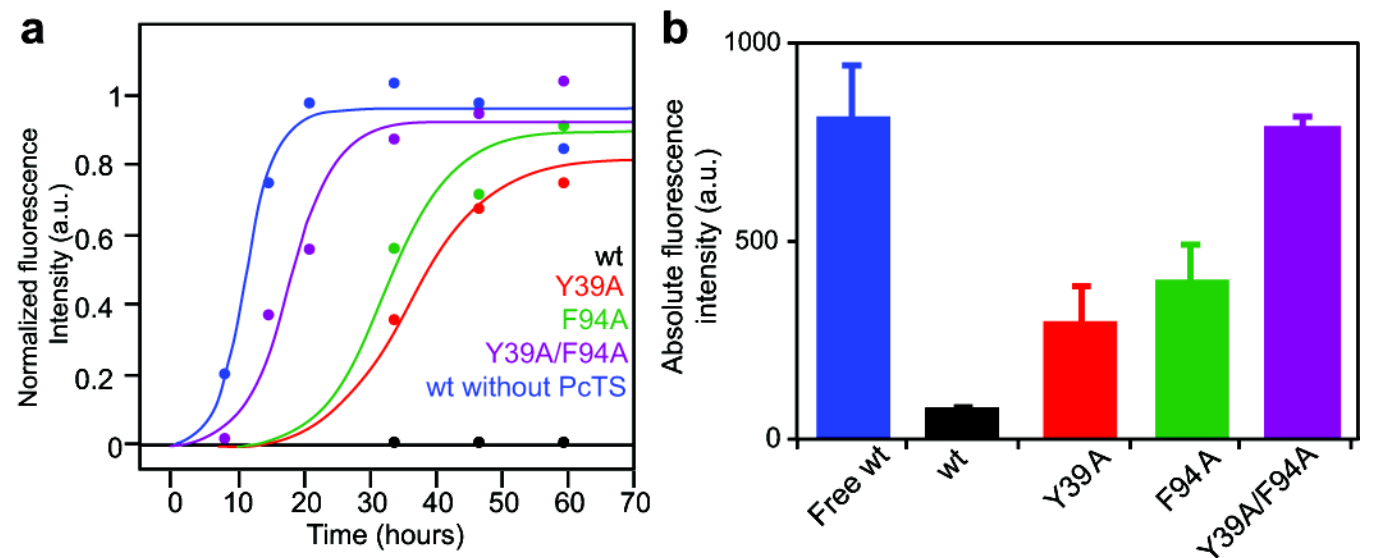

Figure 7 | Sequence specificity of the small molecule-mediated inhibition of amyloid formation of vesicle-bound $\alpha$ Syn. (a) Fibrillization kinetics of $w t \alpha$ Syn and its mutants in the presence of a $\sim 12.5 \mathrm{mM}$ concentration of SUVs. Shown is the normalized ThT intensity for increasing times of incubation in aggregation prone conditions. The concentration of $\alpha$ Syn was $100 \mu \mathrm{M}$ and the molar ratio of PcTS to protein was 15:1. The blue line shows the fibrillization kinetics of wt $\alpha$ Syn in the absence of PcTS. In the presence of PcTS, wt $\alpha$ Syn (black) does not form ThTpositive fibrils. Removing one aromatic residue decreases the PcTS inhibitory effect (green and red lines). Removal of both Y39 and F94 (purple line) abolishes the PcTSmediated aggregation inhibition. The circles represent the average ThT intensity of at least 3 different experiments normalized against their respective maximum values. (b) 
ThT fluorescence after six days of aggregation. The error bars represent the standard error of at least 3 different samples.

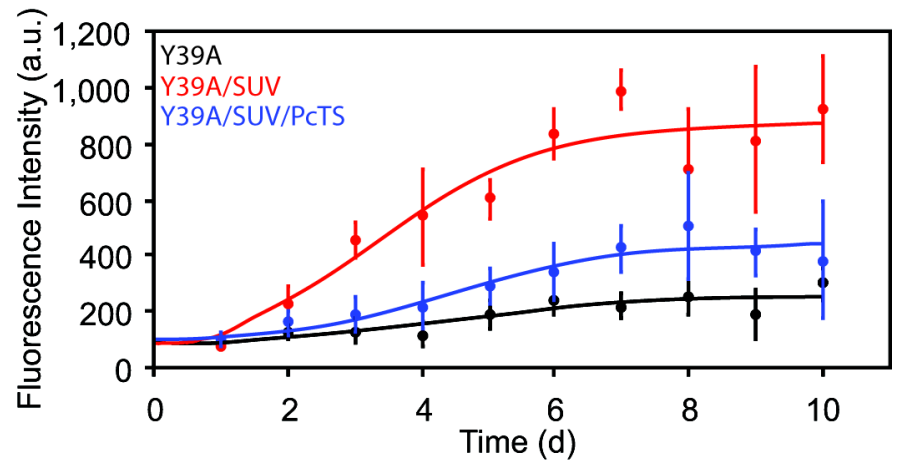

Figure 8 | Fibrillization kinetics of the Y39A mutant of $\alpha$ Syn in solution (black), when bound to membranes (red) and after further addition of PcTS (blue). Shown is the ThT fluorescence intensity after increasing times of incubation in aggregation prone conditions $\left(37^{\circ} \mathrm{C}\right.$ with stirring). The concentration of Y39A $\alpha$ Syn was $100 \mu \mathrm{M}$, that of SUVs $\sim 12.5 \mathrm{mM}$. While little fibrillization was observed for the Y39A mutant of $\alpha$ Syn in solution, it readily aggregates in the presence of SUVs. Moreover, the data show that PcTS is capable of decreasing aggregation of vesiclebound $\alpha$ Syn through an interaction with F94. Error bars represent the standard error for at least three different samples.

PcTS reduces $\alpha$ Syn inclusion formation and cytotoxicity. In order to determine the effect of PcTS on $\alpha$ Syn aggregation and $\alpha$ Syn-induced cytotoxicity, we employed a previously described cell model ${ }^{46}$ that leads to protein inclusion formation positive for $\alpha$ Syn. Addition of 0.1 and $1 \mu \mathrm{M}$ of PcTS to H4 cells expressing $\alpha$ Syn did not modify inclusion formation. In contrast, treatment with $10 \mu \mathrm{M}$ PcTS for $48 \mathrm{~h}$ decreased the percentage of cells with inclusion formation (Fig. 9 a,b)

Next we tested if PcTS would also influence $\alpha$ Syn induced cytotoxicity in the same cell model. Toxicity was determined $48 \mathrm{~h}$ after transfection and start of PcTS 
treatment by determining the release of lactate dehydrogenase (LDH) into the medium. We determined no effect on $\alpha$ Syn toxicity in the vehicle control, but observed a drop in LDH release upon treatment with $10 \mu \mathrm{M}$ PcTS, the same concentration that led to inclusion modulation (Fig. 9c).
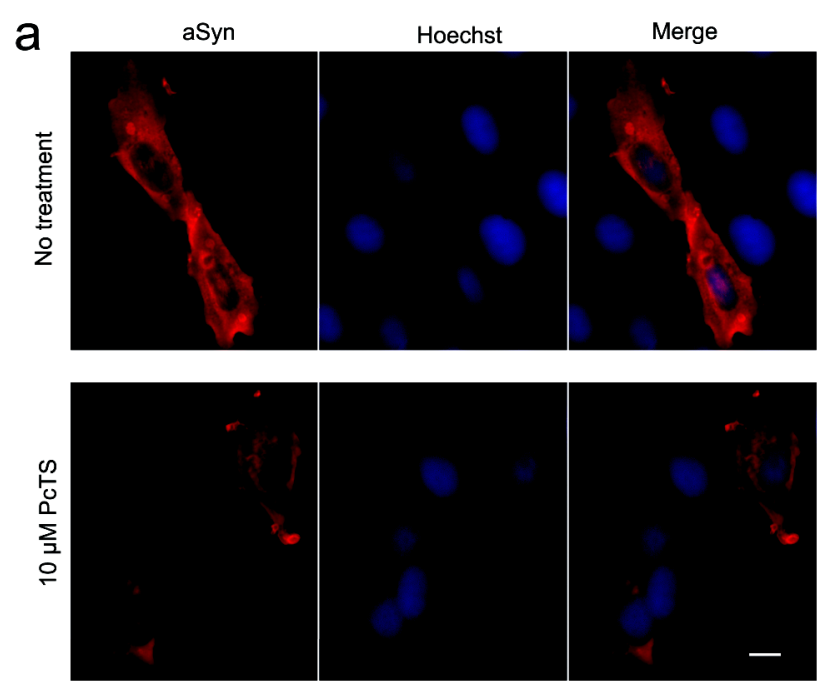

b
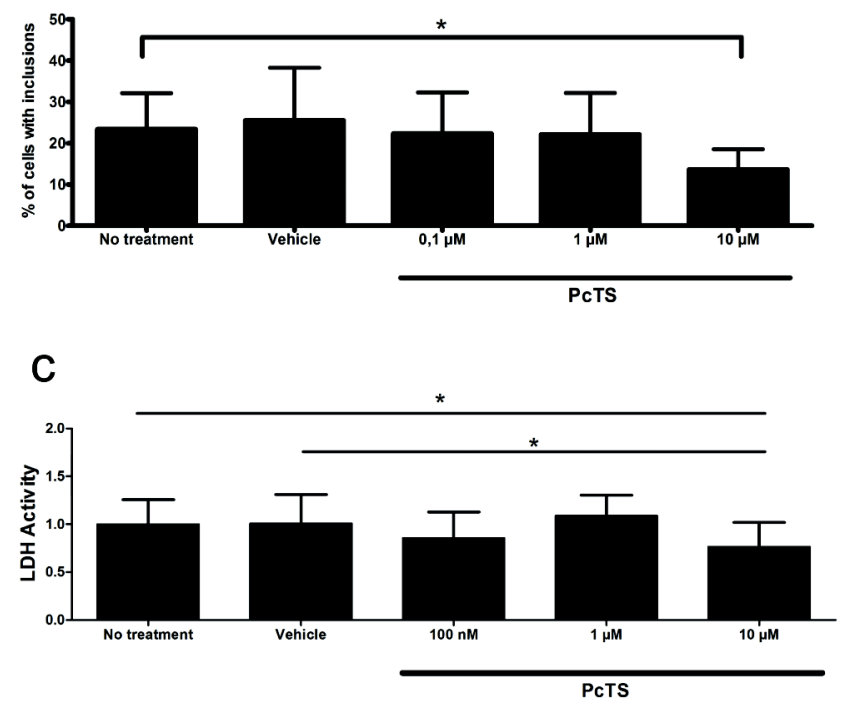

Figure 9 | PcTS reduces $\alpha$ Syn inclusion formation and cytotoxicity. (a) Representative images for $\alpha$ Syn inclusion modulation by $10 \mu \mathrm{M}$ PcTS in H4 cells. Scale bar $=25 \mu \mathrm{m}$. (b) Addition of $10 \mu \mathrm{M}$ to $\mathrm{H} 4$ cells expressing $\alpha$ Syn decreases inclusion formation. No effect can be observed with lower concentrations of PcTS or 
vehicle control. (c) PcTS reduces $\alpha$ Syn-induced cytotoxicity. Addition of $10 \mu \mathrm{M}$ PcTS to H4 cells expressing $\alpha$ Syn decreases release of LDH into the medium, an indicator for cytotoxicity. No effect could be observed with lower concentrations of PcTS or vehicle control. Data represent mean \pm standard deviation of four or seven independent experiments with $\mathrm{P}<0.05$ with paired, two-tailed student's t-test and Oneway ANOVA with post-hoc Bonferroni.

\section{Discussion}

$\alpha$ Syn is important for regulation of synaptic vesicle trafficking ${ }^{8}$ and is found in both soluble and membrane fractions of neuronal cells ${ }^{9,16}$. Consistent with a physiological role of the membrane-bound conformation of $\alpha$ Syn, its N-terminal 100 residues have a high propensity to bind to negatively charged vesicles and fold into an $\alpha$-helical conformation $^{25,47}$. At the same time, $\alpha$ Syn can misfold, aggregate and form fibrils when it is in contact with phospholipid vesicles ${ }^{38}$ pointing also to a pathological role of membrane-bound $\alpha$ Syn.

Aggregation of vesicle-bound $\alpha$ Syn, which has a low surface coverage when compared to other peripheral membrane proteins such as apocytochrome $\mathrm{c}^{44}$, was suggested to occur through a two-state process ${ }^{48}$. Initially, the $\mathrm{N}$-terminus and the NAC domain of $\alpha$ Syn are bound to vesicles in an $\alpha$-helical conformation. Aggregation is initiated by insertion of hydrophobic residues of the NAC domain into the membrane leading to an intermediate conformation. This intermediate conformation is at least partially inserted into the lipid bilayer and is characterized by partial unfolding of the helical conformation and formation of elements of $\beta$-structure ${ }^{48}$. A further step during aggregation of vesicle-bound $\alpha$ Syn is oligomerization through the NAC domain, which occurs either parallel to the unfolding of the helical conformation or 
subsequent to $i^{48}$, followed by formation of amyloid fibrils. Amyloid fibrils formed in the presence of POPC/POPA vesicles closely resembled those formed in the absence of lipid vesicles and were immersed in a network of vesicles with substantially perturbed morphology ${ }^{48}$. A direct attachment of fibrils to SUVs is also supported by studies on the islet amyloid polypeptide, which showed that its fibrils line the surface of distorted phospholipid vesicles, in agreement with the notion that fibril growth at the membrane and membrane damage are physically connected ${ }^{49}$. Our data support the above aggregation model and reveal a way to interfere with aggregation of $\alpha$ Syn in contact with synaptic vesicles. PcTS directly binds to Y39 and F94 of membranebound $\alpha$ Syn. This interaction partially releases the NAC domain from the membrane surface (Figs. 3, 6) such that the helical conformation in the NAC region is less prone to membrane-assisted unfolding, subsequent oligomerization and amyloid formation (Figs. 1, 7, 10).

Inhibition of $\alpha$ Syn aggregation in the membrane-bound state is specific to PcTS, since several other small molecules, which inhibit aggregation of $\alpha$ Syn in solution, did not stop aggregation in the lipid environment (Fig. 10). This suggests that compounds such as curcumin and baicalein primarily act at the stage of soluble oligomer formation, while their affinity to monomeric $\alpha$ Syn is low. In addition, baicalein, selegiline and EGCG interact with liposomes (Supplementary Fig. 2) further decreasing their efficacy in a lipid environment. PcTS, on the other hand, does not bind to liposomes and readily interacts with monomeric $\alpha$ Syn both in the absence and presence of lipid membranes. The green tea polyphenol EGCG also attenuated the formation of $\beta$-sheet rich amyloid fibrils in the presence of vesicles, in agreement with its ability to bind to monomeric polypeptides ${ }^{32}$. However, in contrast to PcTS, EGCG was not able to stabilize the helical, membrane-bound conformation of $\alpha$ Syn 
(Fig. 1). Instead it directs $\alpha$ Syn into heterogeneous off-pathway oligomers ${ }^{32}$. It has also been demonstrated that while EGCG is able to prevent the formation of aggregates and to disrupt already formed fibrils of the islet amyloid polypeptide when used in solution, its ability to do so in a phospholipid interface is greatly diminished ${ }^{50}$. Whether this is the result of a less accessible protein interface for the compound, membrane binding of EGCG or stabilization of the fibril by its interaction with phospholipids is unknown. The effectiveness of small molecules to inhibit aggregation of vesicle-bound proteins might also be related to changes in the aggregation pathway of the protein in the presence of lipids. Whatever the case, the combined data suggest that compounds, which show strong anti-aggregation properties in solution, do not necessarily have the same efficacy when used in the presence of lipids. Thus, it is important to assess the anti-aggregation properties of small molecules in a lipid environment, in particular as disruption of the cellular membrane might be a key mechanism how fibrillar/oligomeric species of $\alpha$ Syn acquire their neurotoxic properties.

The fact that Y39 is essential for aggregation of $\alpha$ Syn in solution ${ }^{34}$ but not when bound to membranes (Fig. 8) shows that the very first stages of aggregation in a membrane environment are different from those that happen in solution. In solution the lack of an aromatic side chain in position 39 would interfere with the selfassembly process of aggregation, whereas in a membrane environment aggregation initially depends on the insertion of hydrophobic residues within the NAC domain and does not need aromatic residues for pathogenic aggregation ${ }^{47}$. 

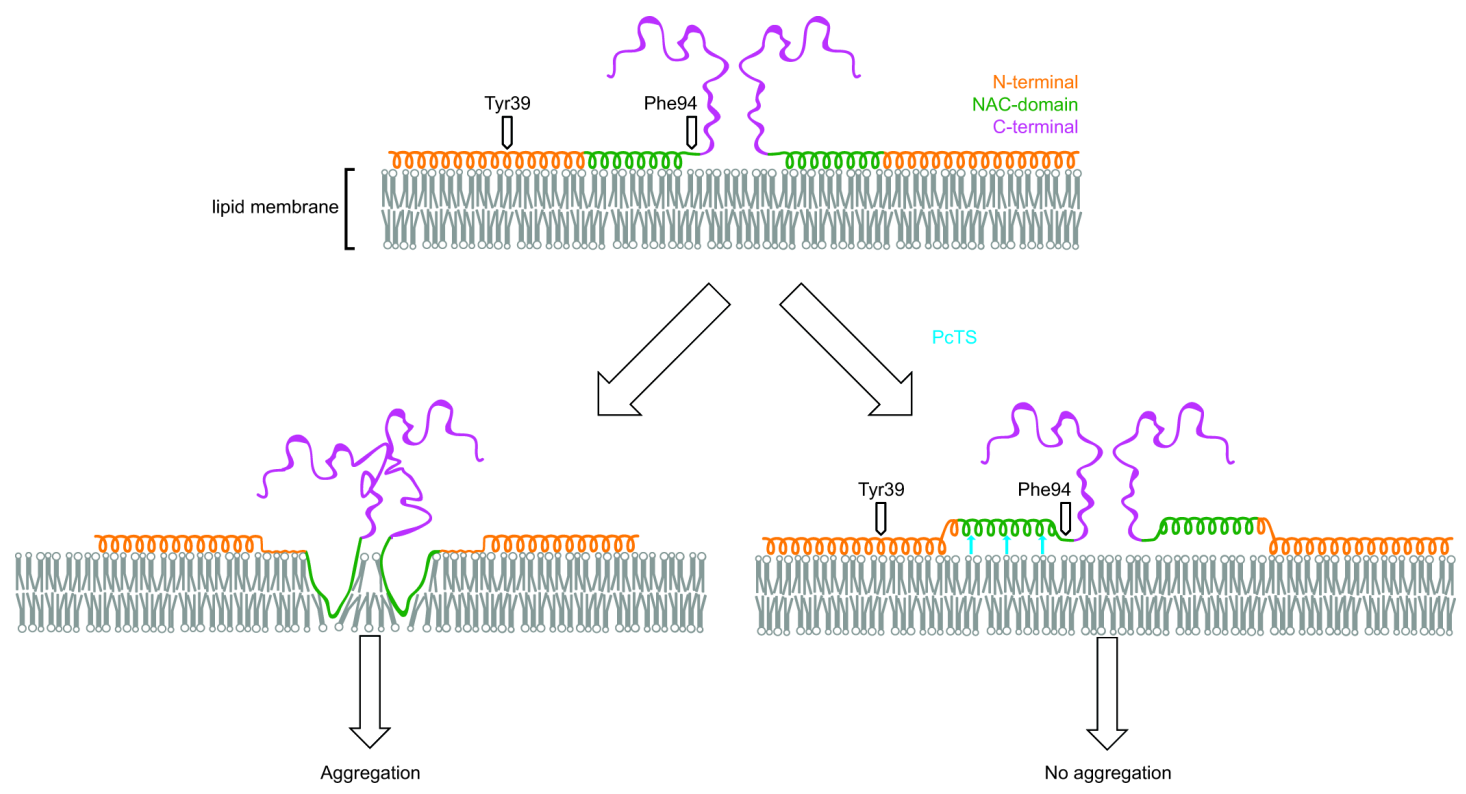

Figure 10 | Small molecule-mediated stabilization of helical vesicle-bound aSyn inhibits pathogenic aggregation. PcTS directly binds to vesicle-bound $\alpha$ Syn, stabilizes its helical conformation and delays misfolding and pathogenic aggregation. In contrast, EGCG, baicalein, curcumin and selegiline inhibit the conversion of the disordered monomeric $\alpha$ Syn into amyloid fibrils, but are not able to stabilize helical, vesicle-bound $\alpha$ Syn. Residues 1-60, 61-95 and 96-140 of $\alpha$ Syn are shown in orange, green and purple. Please see text for further details.

PcTS inhibits the aggregation of prion proteins and has been investigated for its in vivo prophylactic and therapeutic effects in scrapie disease ${ }^{42}$. Inhibition of protease-resistant prion aggregates is achieved through stabilization of the native globular structure of the prion protein ${ }^{51}$. Thus, PcTS is able to stabilize well-folded protein conformations both in solution (prion protein) and on the membrane surface ( $\alpha$ Syn; Fig. 1). PcTS is also likely to interfere with aggregation of membrane-bound $\beta$-synuclein, which has recently been shown to aggregate and cause neuronal loss in the rat brain ${ }^{52}$. $\beta$-synuclein binds membranes similar to $\alpha$ Syn and both Y39 and F94 are conserved ${ }^{53}$. 
Given the wide variety of models that have been proposed to explain the misfolding and aggregation of $\alpha$ Syn, the role of membrane interaction has generated mixed viewpoints. These range from the complete stabilization of the "native state" of $\alpha$ Syn and inhibition of aggregation upon binding of $\alpha$ Syn to vesicles ${ }^{9,16,17,54}$ to the formation of damaging pore-like oligomers formed only in the presence of membranes ${ }^{39}$. As part of these complex processes, $\alpha$ Syn maintains an equilibrium between a free and membrane-bound state and - under stress conditions - is prone to form soluble oligomers and fibrils. However, our study suggests that the mechanism by which $\alpha$ Syn aggregates is very different when it is bound to vesicles than when it is free in solution. We also demonstrate that the detachment of the NAC domain of $\alpha$ Syn from the vesicle surface greatly delays its aggregation and, to the best of our knowledge, this is a unique effect of PcTS. The importance of membrane attachment of the NAC domain for aSyn aggregation is consistent with a recent study, which showed that the NAC domain acts as a sensor of lipid properties and determines the affinity of $\alpha$ Syn membrane binding ${ }^{25}$. Because the anti-aggregation effect of PcTS is much stronger for vesicle-bound $\alpha$ Syn than soluble, disordered $\alpha$ Syn, it is likely that PcTS reduces $\alpha$ Syn inclusion formation and cytotoxicity through binding to membrane attached $\alpha$ Syn. Our study therefore suggests that small molecule-mediated stabilization of the helical membrane-bound state of $\alpha$ Syn provides unique opportunities for inhibiting misfolding and pathogenic aggregation of $\alpha \mathrm{Syn}$ in the physiological environment of a synaptic vesicle. Detailed knowledge about the structural basis of $\alpha$ Syn aggregation, together with a screening procedure for small molecules that is targeted to helical vesicle-bound aSyn, promises new ways to approach Parkinson's disease and related synucleinopathies. 


\section{Methods}

Expression and Purification of $\boldsymbol{\alpha S y n}$ Variants. pT7-7 plasmid encoding for human $w t$ SSyn was kindly provided by the Lansbury Laboratory, Harvard Medical School, Cambridge, MA. A codon replacement was performed for residue Y136 (TAC to TAT) to avoid codon usage problems ${ }^{55}$. The resulting construct was then used as the template for mutagenesis reactions. Mutations were performed by using the QuikChange II site-directed mutagenesis kit (Stratagene) and verified by DNA sequencing. Plasmids containing aSyn variants were expressed in Escherichia coli BL21 (DE3) cells. Protein expression and purification was performed as previously described $^{56}$. For production of ${ }^{15} \mathrm{~N}$-labeled proteins, M9-minimal medium supplemented with ${ }^{15} \mathrm{NH}_{4} \mathrm{Cl}$ (Cambridge Isotope Laboratories) was used.

Preparation of Unilamellar Vesicles. 1-palmitoyl-2-oleoyl phosphatidylcholine (POPC) and 1-palmitoyl-2-oleoyl phosphatidic acid (POPA), 1,2-dioleoyl-sn-glycero3-phosphoethanolamine (DOPE), 1,2-dioleoyl-sn-glycero-3-phospho-L-serine (DOPS) and 1,2-dioleoyl-sn-glycero-3-phosphocholine (DOPC) were obtained from Avanti Polar Lipids. For vesicle formation, a 1:1 ratio of POPC:POPA or a 5:3:2 ratio of DOPE:DOPS:DOPC was dissolved in a $4 \mathrm{ml}$ mixture of chloroform/methanol (1:1 vol/vol), followed by evaporation of all solvents under a stream of $\mathrm{N}_{2}$ gas and lyophilized overnight. The resulting lipid film was hydrated in $50 \mathrm{mM}$ HEPES, 100 $\mathrm{mM} \mathrm{NaCl}, \mathrm{pH} 7.4$ or in $50 \mathrm{mM}$ Na-phosphate buffer (pH 7.4), $100 \mathrm{mM} \mathrm{NaCl}$ to obtain a total lipid concentration of $12.5 \mathrm{mM}$. For preparation of SUVs, the suspension was sonicated at $37 \mathrm{kHz}$ (four times for $10 \mathrm{~min}$ with five min breaks at room temperature or until the sample was transparent) in a glass tube. SUVs were isolated by ultracentrifugation at 55,000 rpm in a Beckman TLA 100.3 rotor for 2 
hours at $298 \mathrm{~K}$. The isolated SUVs had a hydrodynamic diameter of $35 \pm 10 \mathrm{~nm}$ according to dynamic light scattering. LUVs were prepared following the same procedure but with only two rounds of sonication and no ultracentrifugation step. Dynamic light scattering estimated the hydrodynamic diameter of the LUVs as $105 \pm$ $25 \mathrm{~nm}$.

Aggregation of Vesicle-Bound aSyn. PcTS was purchased from MP Biomedicals (MP Biomedicals S.A. Heidelberg, Germany). Selegiline, curcumin, baicalein and EGCG were purchased from Sigma (Sigma-Aldrich Chemie GmbH, Schnelldorf, Germany).

SUVs and LUVs were mixed with purified $\alpha$ Syn variants at a 125:1 molar ratio of phospholipid to protein in $50 \mathrm{mM}$ HEPES, $100 \mathrm{mM} \mathrm{NaCl} \mathrm{pH} \mathrm{7.4,0.02 \%}$ sodium azide (with final concentrations of $12.5 \mathrm{mM}$ of phospholipids and $100 \mu \mathrm{M}$ for aSyn). All compounds were solubilized in a $500 \mathrm{mM}$ stock solution of dimethyl sulfoxide (DMSO) and added to the working solution for final concentrations of 0.1 , 0.5 and $1.5 \mathrm{mM}$ (giving 1:1, 5:1 and 15:1 compound to protein ratios). Subsequently, samples were subjected to aggregation-prone conditions $\left(37^{\circ} \mathrm{C} \sim 300 \mathrm{rpm}\right)$ in low binding protein ${ }^{\mathrm{TM}} 1.5 \mathrm{ml}$ Eppendorf tubes over a period of 10 days. After different incubation times, a $20 \mu \mathrm{l}$ aliquot of the sample was mixed with $1 \mathrm{ml}$ of a $100 \mathrm{mM}$ ThT solution. Fluorescence emission was measured between 460 and $600 \mathrm{~nm}$ on a Varian Cary Eclipse fluorescence spectrophotometer (Agilent Technologies) with excitation wavelength of $442 \mathrm{~nm}$ at $20^{\circ} \mathrm{C}$. The averaged data points were fitted to a sigmoidal equation (data are presented as mean \pm standard error, $\mathrm{n}=3$ ). 
CD Spectroscopy. Far UV-CD measurements were performed at $20{ }^{\circ} \mathrm{C}$ on a Chirascan (Applied Photophysics, UK) circular dichroism spectrometer, using a protein concentration of $10 \mu \mathrm{M}$ in a quartz cuvette with $0.1 \mathrm{~cm}$ light-path. For vesiclebound $\alpha$ Syn, SUVs or LUVs were added to a final concentration of $1.5 \mathrm{mM}$. Each experiment was repeated at least twice. Baseline correction was performed with the same buffer. Data were expressed as mean residue ellipticity (degree $\mathrm{cm}^{2} \mathrm{dmol}^{-1}$ ). Estimation of secondary structure content was performed by neural-network analysis using the program K2D available at the DichroWeb server ${ }^{57}$.

NMR Spectroscopy. NMR samples contained $0.1 \mathrm{mM}{ }^{15} \mathrm{~N}$-labeled $w t$ or mutant $\alpha$ Syn in $50 \mathrm{mM}$ HEPES buffer, $100 \mathrm{mM} \mathrm{NaCl}$, pH 7.4 or $50 \mathrm{mM}$ Na-phosphate buffer, 100 $\mathrm{mM} \mathrm{NaCl}$ at $\mathrm{pH} 7.4$ and $90 \% \mathrm{H}_{2} \mathrm{O} / 10 \% \mathrm{D}_{2} \mathrm{O}$. NMR experiments were recorded on a Bruker Avance $600 \mathrm{MHz}$ spectrometer. The temperature was set to $15{ }^{\circ} \mathrm{C}$. Data processing was performed using the software packages Topspin (Bruker) and CCPN Analysis $^{58}$.

To probe the binding of $\alpha$ Syn variants to vesicles, $2 \mathrm{D}{ }^{15} \mathrm{~N}-{ }^{1} \mathrm{H}$ HSQC spectra were recorded in the presence of either $12.5 \mathrm{mM}$ SUV or LUVs. The same stock solution of compounds in DMSO was used for the NMR experiments in the same ratios, so the final DMSO concentration never surpassed $1 \%$ of the total volume.

${ }^{15} \mathrm{~N}-{ }^{1} \mathrm{H}$ HSQC amide cross-peaks affected during compound addition were identified by comparison of their chemical shift values with those of the same crosspeaks in the data set of samples lacking the compound. Perturbations in the chemical shift values for ${ }^{1} \mathrm{H}$ and ${ }^{15} \mathrm{~N}$ were calculated as $\left[\left(\Delta \delta^{1} \mathrm{H}\right)^{2}+\left(\Delta \delta^{15} \mathrm{~N} / 10\right)^{2}\right]^{1 / 2}$. Intensity profiles $\left(\mathrm{I} / \mathrm{I}_{0}\right)$ were obtained by comparing intensities of ${ }^{15} \mathrm{~N}-{ }^{1} \mathrm{H}$ HSQC cross-peaks observed in the presence (I) and absence $\left(\mathrm{I}_{0}\right)$ of compound. 
${ }^{15} \mathrm{~N}$ TROSY experiments for $R_{2}{ }^{\mathrm{T}}$ measurement (modified from ${ }^{59}$ ) were recorded at $30{ }^{\circ} \mathrm{C}$ on a Bruker $600 \mathrm{MHz}$ spectrometer as interleaved experiments with six $T_{2}$ delays (10, 40, 80, 150, 250 and $\left.400 \mathrm{~ms}\right)$. Samples contained $250 \mu \mathrm{M}$ protein and $1.5 \mathrm{mM}$ SUVs (1:6 protein-to-lipid ratio) in $50 \mathrm{mM}$ HEPES buffer, $100 \mathrm{mM}$ $\mathrm{NaCl}$ at $\mathrm{pH} 7.4$ with $10 \% \mathrm{D}_{2} \mathrm{O}$. Single exponential decay curves were fitted to the signal intensity of each cross-peak throughout the different delay times with the function $\mathrm{y}=\mathrm{A} e^{(-\mathrm{Bx})}+\mathrm{C}$, where $\mathrm{B}$ is the $R_{2}{ }^{\mathrm{T}}$ value. Error bars represent the fitting errors.

Transmission Electron Microscopy. A solution containing protein was applied to glow-discharged carbon coated grids and stained with $1 \%$ uranyl acetate. Images were taken in a Philips CM120 electron microscope (Philips Inc.) at a defocus of $2.3 \mu \mathrm{m}$ using a TemCam 224A slow scan CCD camera (TVIPS, Gauting, Germany).

Cell culture and transfection. $\mathrm{H} 4$ neuroglioma cells were maintained at $5 \% \mathrm{CO}_{2}$ and $37^{\circ} \mathrm{C}$ in Optimem medium (Gibco) supplemented with $10 \%$ Fetal Calf Serum and $1 \%$ Penicillin Streptomycin. One day prior to transfection cells were seeded in 24-well plates. Cells were transiently transfected with aSyn using Metafectene (Biontex) according to manufacturer's instructions. PcTS compound in DMSO was added in concentrations of $100 \mathrm{nM}, 1 \mu \mathrm{M}$ and $10 \mu \mathrm{M}$ to the cells. $\alpha$ Syn expressing cells were treated with DMSO to exclude vehicle effects.

Immunocytochemistry. Cells were fixed with 4\% Paraformaldehyde (PFA) in PBS 48 hours after transfection and start of PcTS treatment. For permeabilization cells were subsequently treated with $0.1 \%$ Triton X-100 and blocked with $1.5 \%$ Bovine 
Serum Albumin (BSA) in PBS. Cells were then incubated with anti $\alpha$ Syn antibody (BD $610787,1: 2000$ ) either 4 hours at room temperature or overnight at $4^{\circ} \mathrm{C}$ and 3 hours in Alexa Fluor donkey anti-mouse 555 (Invitrogen A31570, 1:2000). Cells were further stained with Hoechst (Molecular Probes 33258) for 5-10 minutes. Images were captured using a Leica DMI 6000B.

LDH activity. LDH activity was measured via release of LDH into the culture medium with the Cytotoxicity Detection Kit (LDH) (Roche). Basic LDH release was measured in non-transfected cells, total LDH release was measured by cell lysis in $2 \%$ Triton X-100. Absorbance was measured with the Infinite M200 PRO (Tecan) plate reader at $490 \mathrm{~nm}$. Experimental values were calculated in percentages of total LDH release and normalized to tranfection with SynT without treatment. Statistical analysis was conducted using GraphPad Prism 4.03 and included paired, two-tailed student's ttest.

\section{References}

1. Lees, A. J., Hardy, J. \& Revesz, T. Parkinson's disease. Lancet 373, 2055-2066 (2009).

2. Goedert, M., Spillantini, M. G., Del Tredici, K. \& Braak, H. 100 years of Lewy pathology. Nat Rev Neurol 9, 13-24 (2012).

3. Hellstrand, E., Nowacka, A., Topgaard, D., Linse, S. \& Sparr, E. Membrane Lipid Co-Aggregation with $\alpha$-Synuclein Fibrils. PLoS ONE 8, e77235 (2013).

4. Tõugu, V.\& Palumaa, P. Coordination of zinc ions to the key proteins of neurodegenerative diseases: A $\beta$, APP, $\alpha$-synuclein and PrP. Coord. Chem. Rev. 256, 2219-2224 (2012).

5. Binolfi, A. et al. Interaction of alpha-synuclein with divalent metal ions reveals key differences: a link between structure, binding specificity and fibrillation enhancement. J. Am. Chem. Soc. 128, 9893-9901 (2006).

6. Uversky, V. N., Li, J. \& Fink, A. L. Metal-triggered Structural Transformations, Aggregation, and Fibrillation of Human $\alpha$-Synuclein. A 
possible molecular link between Parkinson's disease and heavy metal exposure. J. Biol. Chem. 276, 44284-44296 (2001).

7. Braun, A. R. et al. $\alpha$-Synuclein Induces Both Positive Mean Curvature and Negative Gaussian Curvature in Membranes. J. Am. Chem. Soc. 134, 26132620 (2012).

8. Burre, J. et al. Alpha-synuclein promotes SNARE-complex assembly in vivo and in vitro. Science 329, 1663-1667 (2010).

9. Burre, J. et al. Properties of native brain $\alpha$-synuclein. Nature 498, E4-6discussion E6-7 (2013).

10. Choi, B.-K. et al. Large $\alpha$-synuclein oligomers inhibit neuronal SNAREmediated vesicle docking. Proc. Natl. Acad. Sci. U.S.A. 110, 4087-4092 (2013).

11. Bertoncini, C. W. et al. Release of long-range tertiary interactions potentiates aggregation of natively unstructured alpha-synuclein. Proc. Natl. Acad. Sci. U.S.A. 102, 1430-1435 (2005).

12. Dedmon, M. M., Lindorff-Larsen, K., Christodoulou, J., Vendruscolo, M. \& Dobson, C. M. Mapping Long-Range Interactions in $\alpha$-Synuclein using SpinLabel NMR and Ensemble Molecular Dynamics Simulations. J. Am. Chem. Soc. 127, 476-477 (2005).

13. Eliezer, D., Kutluay, E., Bussell, R., Jr \& Browne, G. Conformational properties of $\alpha$-synuclein in its free and lipid-associated states. J. Mol. Biol. 307, 1061-1073 (2001).

14. Fauvet, B. et al. $\alpha$-Synuclein in central nervous system and from erythrocytes, mammalian cells, and Escherichia coli exists predominantly as disordered monomer. J. Biol. Chem. 287, 15345-15364 (2012).

15. Lashuel, H. A., Overk, C. R., Oueslati, A. \& Masliah, E. The many faces of $\alpha-$ synuclein: from structure and toxicity to therapeutic target. Nat. Rev. Neurosci. 14, 38-48 (2013).

16. Bartels, T., Choi, J. G. \& Selkoe, D. J. $\alpha$-Synuclein occurs physiologically as a helically folded tetramer that resists aggregation. Nature 477, 107-110 (2011).

17. Wang, W. et al. A soluble $\alpha$-synuclein construct forms a dynamic tetramer. Proc. Natl. Acad. Sci. U.S.A. 108, 17797-17802 (2011).

18. Davidson, W. S., Jonas, A., Clayton, D. F. \& George, J. M. Stabilization of $\alpha-$ synuclein secondary structure upon binding to synthetic membranes. J. Biol. Chem. 273, 9443-9449 (1998).

19. Auluck, P. K., Caraveo, G. \& Lindquist, S. $\alpha$-Synuclein: Membrane Interactions and Toxicity in Parkinson's Disease. Annu. Rev. Cell Dev. Biol. 26, 211-233 (2010).

20. Jo, E., McLaurin, J., Yip, C. M., St George-Hyslop, P. H. \& Fraser, P. E. alphaSynuclein membrane interactions and lipid specificity. J. Biol. Chem. 275, 34328-34334 (2000).

21. Georgieva, E. R., Ramlall, T. F., Borbat, P. P., Freed, J. H. \& Eliezer, D. Membrane-Bound $\alpha$-Synuclein Forms an Extended Helix: Long-Distance Pulsed ESR Measurements Using Vesicles, Bicelles, and Rodlike Micelles. $J$. Am. Chem. Soc. 130, 12856-12857 (2008).

22. Trexler, A. J. \& Rhoades, E. Alpha-synuclein binds large unilamellar vesicles as an extended helix. Biochemistry 48, 2304-2306 (2009).

23. Ulmer, T. S., Bax, A., Cole, N. B. \& Nussbaum, R. L. Structure and dynamics of micelle-bound human alpha-synuclein. J. Biol. Chem. 280, 9595-9603 (2005). 
24. Bodner, C. R., Dobson, C. M. \& Bax, A. Multiple tight phospholipid-binding modes of alpha-synuclein revealed by solution NMR spectroscopy. J. Mol. Biol. 390, 775-790 (2009).

25. Fusco, G. et al. Direct observation of the three regions in $\alpha$-synuclein that determine its membrane-bound behaviour. Nat. Commun. 5, 3827 (2014).

26. Der-Sarkissian, A., Jao, C. C., Chen, J. \& Langen, R. Structural organization of alpha-synuclein fibrils studied by site-directed spin labeling. J. Biol. Chem. 278, 37530-37535 (2003).

27. Winner, B. et al. In vivo demonstration that alpha-synuclein oligomers are toxic. Proc. Natl. Acad. Sci. U.S.A. 108, 4194-4199 (2011).

28. Karpinar, D. P. et al. Pre-fibrillar alpha-synuclein variants with impaired betastructure increase neurotoxicity in Parkinson's disease models. EMBO J. 28, 3256-3268 (2009).

29. Lansbury, P. T., Jr \& Lashuel, H. A. A century-old debate on protein aggregation and neurodegeneration enters the clinic. Nature 443, 774-779 (2006).

30. Zhu, M. et al. The flavonoid baicalein inhibits fibrillation of alpha-synuclein and disaggregates existing fibrils. J. Biol. Chem. 279, 26846-26857 (2004).

31. Ahmad, B. \& Lapidus, L. J. Curcumin prevents aggregation in $\alpha$-synuclein by increasing reconfiguration rate. J. Biol. Chem. 287, 9193-9199 (2012).

32. Ehrnhoefer, D. E. et al. EGCG redirects amyloidogenic polypeptides into unstructured, off-pathway oligomers. Nat. Struct. Mol. Biol. 15, 558-566 (2008).

33. Braga, C. A. et al. The Anti-Parkinsonian Drug Selegiline Delays the Nucleation Phase of $\alpha$-Synuclein Aggregation Leading to the Formation of Nontoxic Species. J. Mol. Biol. 405, 254-273 (2011).

34. Lamberto, G. R. et al. Structural and mechanistic basis behind the inhibitory interaction of PcTS on $\alpha$-synuclein amyloid fibril formation. Proc. Natl. Acad. Sci. U.S.A. 106, 21057-21062 (2009).

35. Chung, C. Y. et al. Identification and rescue of $\alpha$-synuclein toxicity in Parkinson patient-derived neurons. Science 342, 983-987 (2013).

36. Tardiff, D. F., Tucci, M. L., Caldwell, K. A., Caldwell, G. A. \& Lindquist, S. Different 8-hydroxyquinolines protect models of TDP-43 protein, $\alpha$-synuclein, and polyglutamine proteotoxicity through distinct mechanisms. J. Biol. Chem. 287, 4107-4120 (2012).

37. Kritzer, J. A. et al. Rapid selection of cyclic peptides that reduce alphasynuclein toxicity in yeast and animal models. Nat. Chem. Biol. 5, 655-663 (2009).

38. Perrin, R. J., Woods, W. S., Clayton, D. F. \& George, J. M. Exposure to long chain polyunsaturated fatty acids triggers rapid multimerization of synucleins. J. Biol. Chem. 276, 41958-41962 (2001).

39. Lashuel, H. A. et al. $\alpha$-Synuclein, especially the Parkinson's disease-associated mutants, forms pore-like annular and tubular protofibrils. J. Mol. Biol. 322, 1089-1102 (2002).

40. Lee, E.-N. et al. Phthalocyanine tetrasulfonates affect the amyloid formation and cytotoxicity of alpha-synuclein. Biochemistry 43, 3704-3715 (2004).

41. Uversky, V. N., Li, J. \& Fink, A. L. Evidence for a partially folded intermediate in alpha-synuclein fibril formation. J. Biol. Chem. 276, $10737-$ 10744 (2001).

42. Priola, S. A., Raines, A. \& Caughey, W. Prophylactic and therapeutic effects of 
phthalocyanine tetrasulfonate in scrapie-infected mice. J. Infect. Dis. 188, 699705 (2003).

43. Bieschke, J. et al. EGCG remodels mature alpha-synuclein and amyloid-beta fibrils and reduces cellular toxicity. Proc. Natl. Acad. Sci. U.S.A. 107, 77107715 (2010).

44. Pfefferkorn, C. M. \& Lee, J. C. Tryptophan probes at the alpha-synuclein and membrane interface. J. Phys. Chem. B 114, 4615-4622 (2010).

45. Bodner, C. R., Maltsev, A. S., Dobson, C. M. \& Bax, A. Differential Phospholipid Binding of $\alpha$-Synuclein Variants Implicated in Parkinson's Disease Revealed by Solution NMR Spectroscopy - Biochemistry (ACS Publications). Biochemistry (2010).

46. McLean, P. J., Kawamata, H. \& Hyman, B. T. Alpha-synuclein-enhanced green fluorescent protein fusion proteins form proteasome sensitive inclusions in primary neurons. Neuroscience 104, 901-912 (2001).

47. Perrin, R. J., Woods, W. S., Clayton, D. F. \& George, J. M. Interaction of human alpha-Synuclein and Parkinson's disease variants with phospholipids. Structural analysis using site-directed mutagenesis. J. Biol. Chem. 275, 3439334398 (2000).

48. Comellas, G., Lemkau, L. R., Zhou, D. H., George, J. M. \& Rienstra, C. M. Structural Intermediates during $\alpha$-Synuclein Fibrillogenesis on Phospholipid Vesicles. J. Am. Chem. Soc. 134, 5090-5099 (2012).

49. Engel, M. F. M. et al. Membrane damage by human islet amyloid polypeptide through fibril growth at the membrane. Proc. Natl. Acad. Sci. U.S.A. 105, 6033-6038 (2008).

50. Engel, M. F. M. et al. The polyphenol EGCG inhibits amyloid formation less efficiently at phospholipid interfaces than in bulk solution. J. Am. Chem. Soc. 134, 14781-14788 (2012).

51. Dee, D. R. et al. Phthalocyanine tetrasulfonates bind to multiple sites on natively-folded prion protein. Biochim. Biophys. Acta 1824, 826-832 (2012).

52. Taschenberger, G. et al. $\beta$-synuclein aggregates and induces neurodegeneration in dopaminergic neurons. Ann. Neurol. 74, 109-118 (2013).

53. Sung, Y.-H. \& Eliezer, D. Secondary structure and dynamics of micelle bound $\beta$ - and $\gamma$-synuclein. Protein Sci. 15, 1162-1174 (2006).

54. Högen, T. et al. Two different binding modes of $\alpha$-synuclein to lipid vesicles depending on its aggregation state. Biophys. J. 102, 1646-1655 (2012).

55. Kuwahara, T. et al. Familial Parkinson mutant alpha-synuclein causes dopamine neuron dysfunction in transgenic Caenorhabditis elegans. J. Biol. Chem. 281, 334-340 (2006).

56. Hoyer, W. et al. Dependence of alpha-synuclein aggregate morphology on solution conditions. J. Mol. Biol. 322, 383-393 (2002).

57. Whitmore, L. \& Wallace, B. A. Protein secondary structure analyses from circular dichroism spectroscopy: Methods and reference databases. Biopolymers 89, 392-400 (2008).

58. Vranken, W. F. et al. The CCPN data model for NMR spectroscopy: Development of a software pipeline. Proteins 59, 687-696 (2005).

59. Lakomek, N.-A., Ying, J. \& Bax, A. Measurement of $15 \mathrm{~N}$ relaxation rates in perdeuterated proteins by TROSY-based methods. J. Biomol. NMR 53, 209221 (2012).

60. Heise, H. et al. Molecular-level secondary structure, polymorphism, and dynamics of full-length alpha-synuclein fibrils studied by solid-state NMR. 
Proc. Natl. Acad. Sci. U.S.A. 102, 15871-15876 (2005).

\section{Acknowledgements}

This work was supported by the Max Planck Society, the European Community's Seventh Framework Programme FP7/2009 under Grant Agreement 238316 and the Cluster of Excellence and DFG Research Center Nanoscale Microscopy and Molecular Physiology of the Brain (to M.Z.). C.O.F. thanks Agencia Nacional de Promoción Científica y Tecnológica, the Max Planck Society, and the Alexander von Humboldt Foundation for financial support. We thank Dr. Martin Schwalbe for discussions and Dr. Dietmar Riedel for electron microscopy.

\section{Author contributions}

M.Z. supervised the project. L.F.-O. performed NMR experiments, aggregation studies, CD spectroscopy and prepared vesicles. S.E.E and T.F.O. performed cell experiments. C.O.F. analyzed data. M.P., K.G. and S.B. prepared protein samples. L.F.-O. and M.Z. co-wrote the paper. 


\section{Supplementary information}

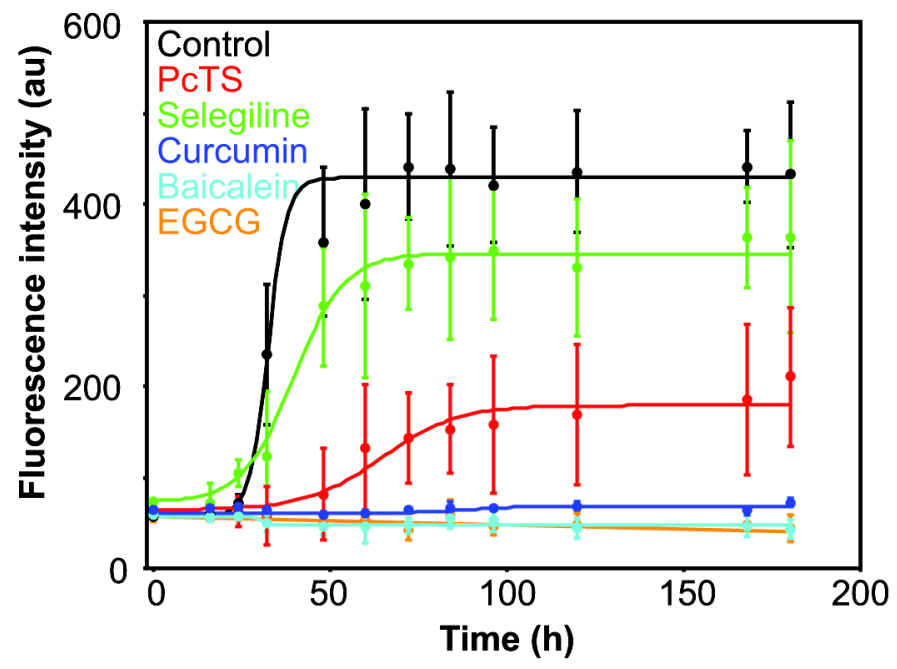

Supplementary Figure 1 | Influence of selected small molecules on fibril formation of disordered $\boldsymbol{\alpha S y n}$ in solution. Fibril formation kinetics in aggregation prone conditions $\left(37^{\circ} \mathrm{C}\right.$ with stirring). The concentration of $\alpha$ Syn was $100 \mu \mathrm{M}$. 15fold excess of compound over protein was used. The final DMSO concentration was $1 \%$ of the total volume. Error bars represent \pm standard deviation from the mean of at least 3 different experiments.

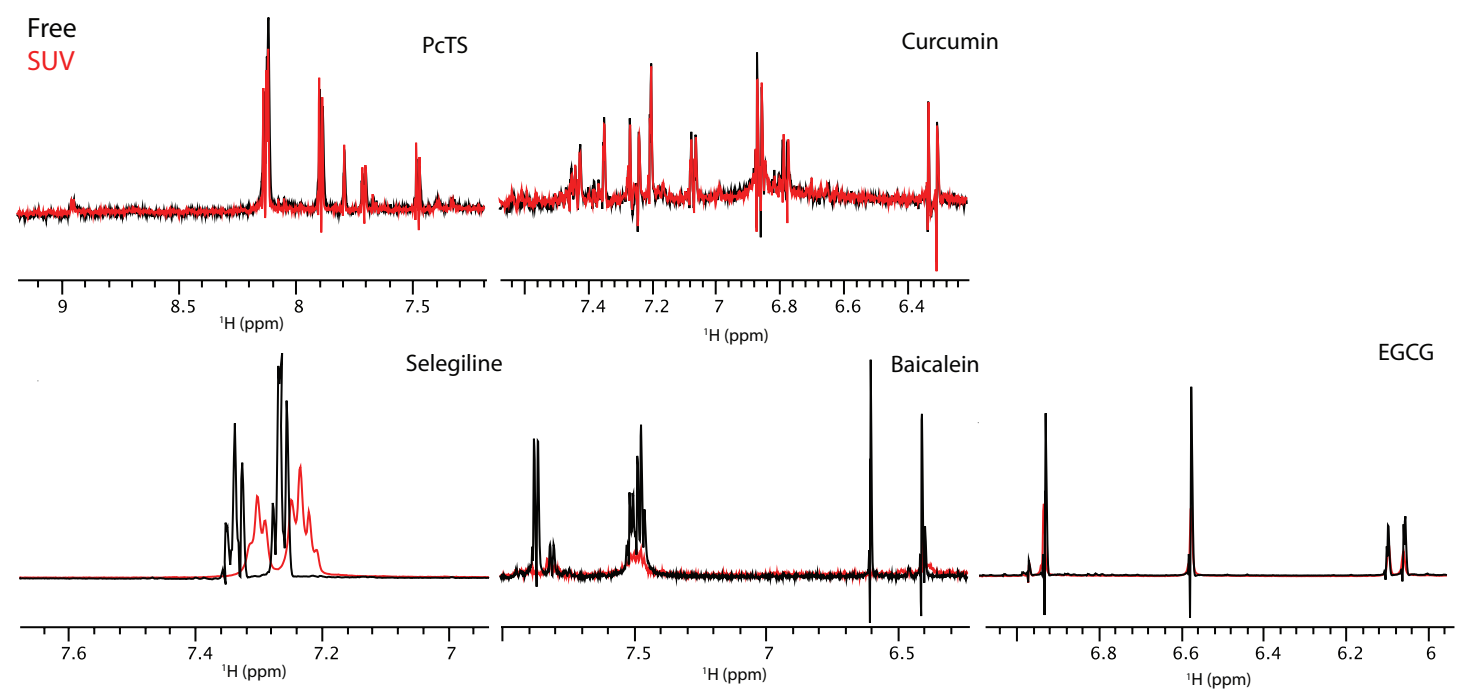

Supplementary Figure 2 | Selected region of the ${ }^{1} \mathrm{H}$ NMR spectra of the small molecules PcTS, curcumin, selegiline, baicalein and EGCG in the absence (black) and presence (red) of SUVs. Upon addition of vesicles, only the resonances of PcTS and curcumin remained unperturbed. Selegiline, baicalein and EGCG interact with vesicles and therefore show changes in NMR signal position and intensity. The final DMSO concentration was $1 \%$ of the total volume in $50 \mathrm{mM}$ HEPES buffer, $100 \mathrm{mM}$ $\mathrm{NaCl}, \mathrm{pH}$ 7.4. NMR spectra were recorded at $288 \mathrm{~K}$. Lipid and compound concentrations were $12.5 \mathrm{mM}$ and $1.5 \mathrm{mM}$, respectively. 

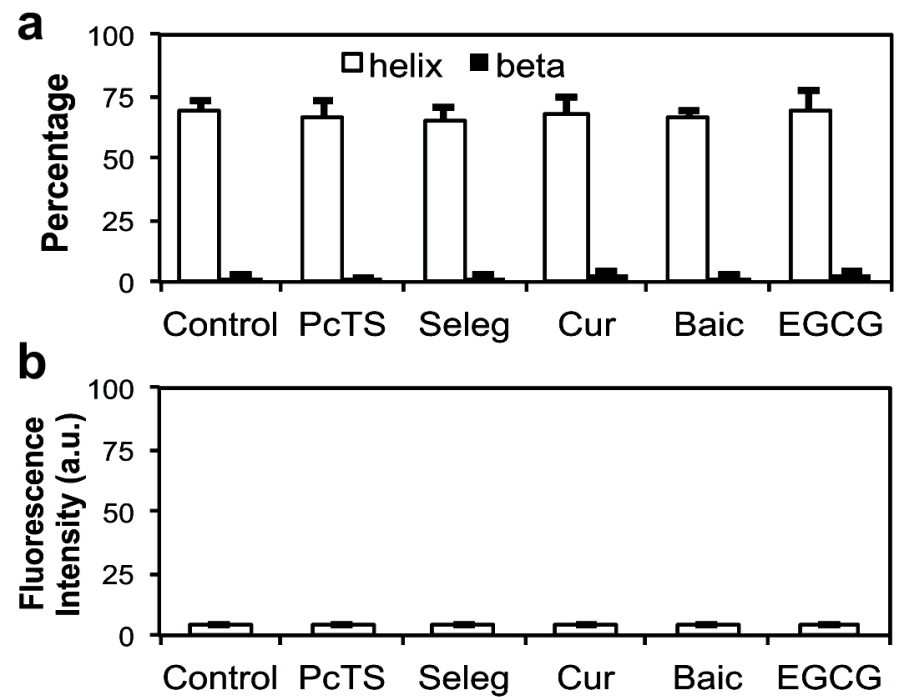

Supplementary Figure 3 | Conformational properties of aSyn prior to aggregation. (a) Secondary structure content of $100 \mu \mathrm{M}$ fresh $\alpha$ Syn bound to 12.5 $\mathrm{mM}$ SUVs. Note that addition of a 15 -fold excess of any of the compounds did not affect the binding of $\alpha$ Syn to SUVs and its vesicle-induced folding into a $\alpha$-helical conformation. (b) ThT fluorescence signal before aggregation. Average values of at least 3 different samples.

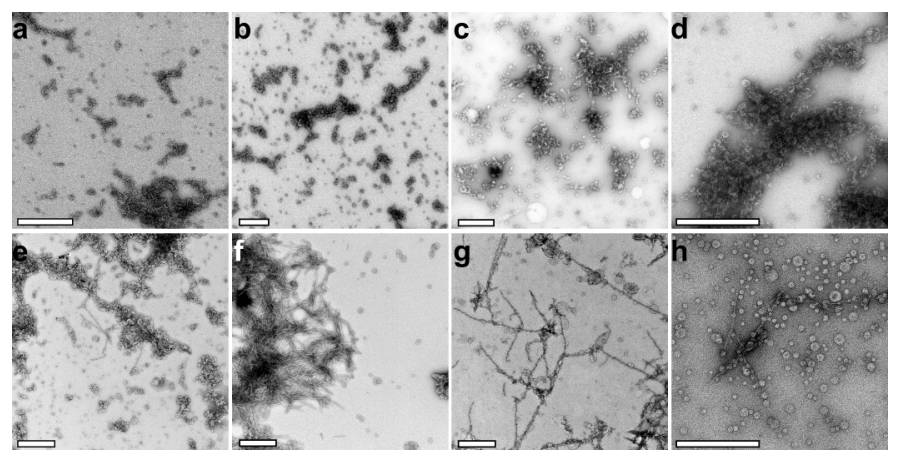

Supplementary Figure 4 | Electron micrographs of SUV-bound $\alpha$ Syn before (a-d) and after incubation for 10 days in aggregation-prone conditions (e-h) in the presence of selegiline $(a, e)$, curcumin $(b, f)$, baicalein $(c, g)$ and EGCG $(d, h)$. White bars represent $500 \mathrm{~nm}$. 


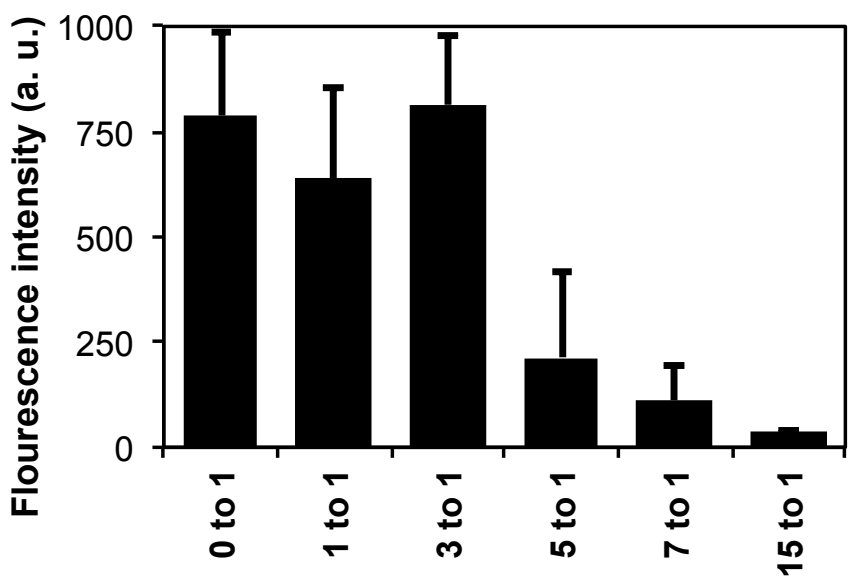

Supplementary Figure 5 | Influence of the concentration of PcTS on the aggregation of membrane-bound $\boldsymbol{\alpha S y n}$. Shown is the ThT fluorescence intensity after 10 days of incubation in aggregation prone conditions $\left(37^{\circ} \mathrm{C}\right.$ with stirring). The concentration of $\alpha$ Syn was $100 \mu \mathrm{M}$, that of SUVs $\sim 12.5 \mathrm{mM}$. The molar ratio of PcTS to protein ranged from 0:1 (no PcTS) to 15:1 (1.5 mM PcTS). Average values of at least 3 different samples. 


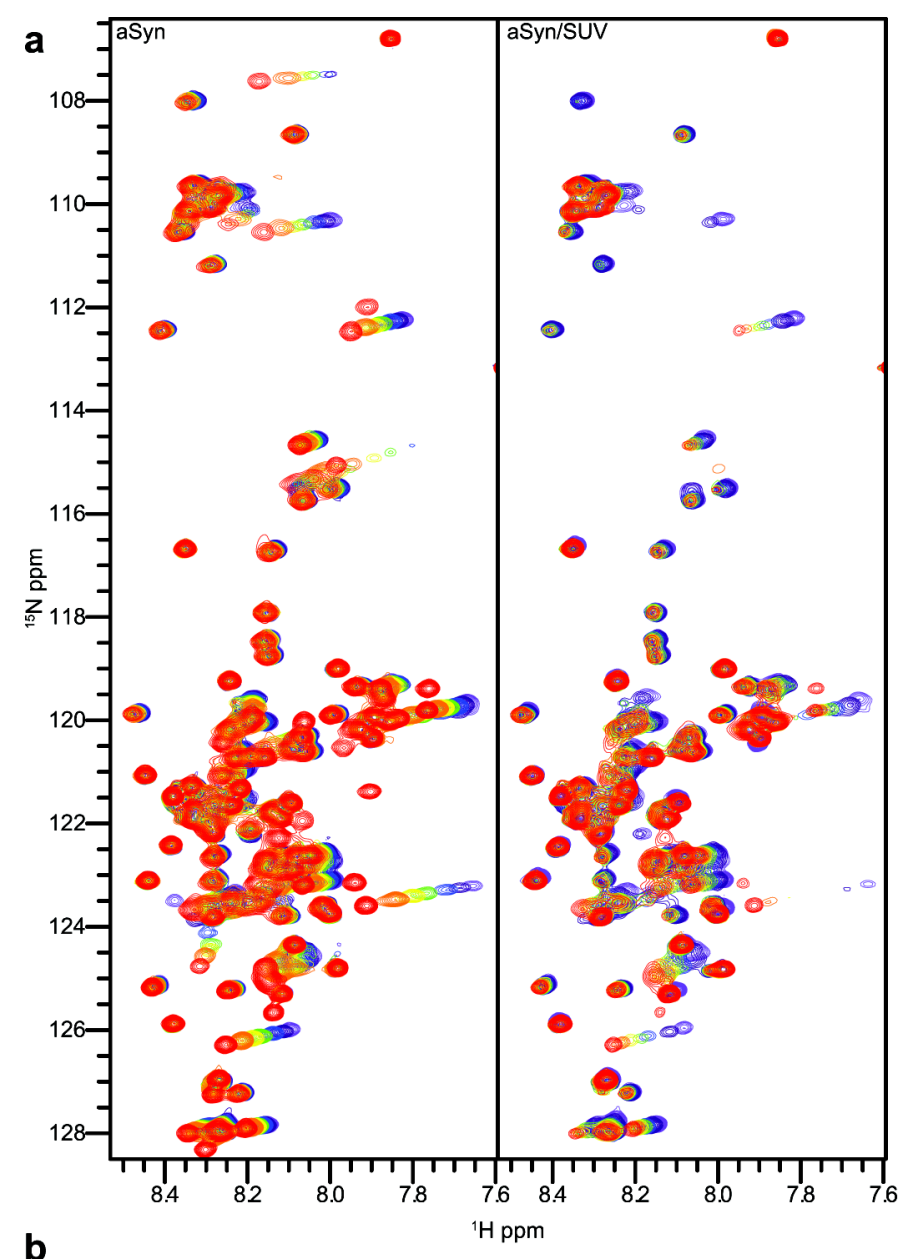

b
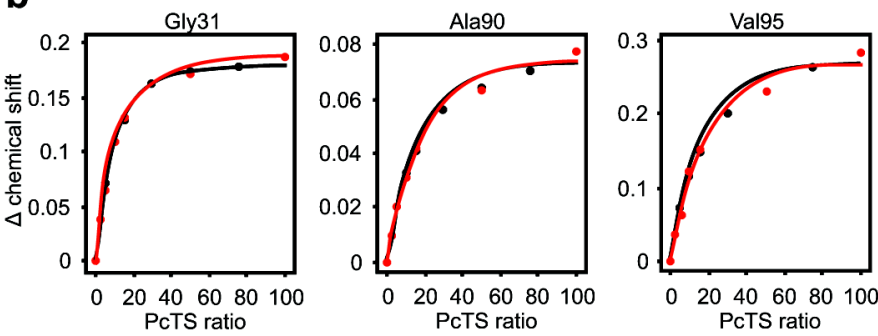

Supplementary Figure 6 | The presence of SUVs does not affect the affinity of PcTS to $\alpha$ Syn. (a) ${ }^{15} \mathrm{~N}-{ }^{1} \mathrm{H}$ HSQC spectra of free (left panel) and SUV-bound (right panel) $\alpha$ Syn at increasing concentrations of PcTS (from red to blue). (b) Chemical shift changes as a function of PcTS concentration for selected residues in the presence (red line) and absence (black line) of SUVs. 


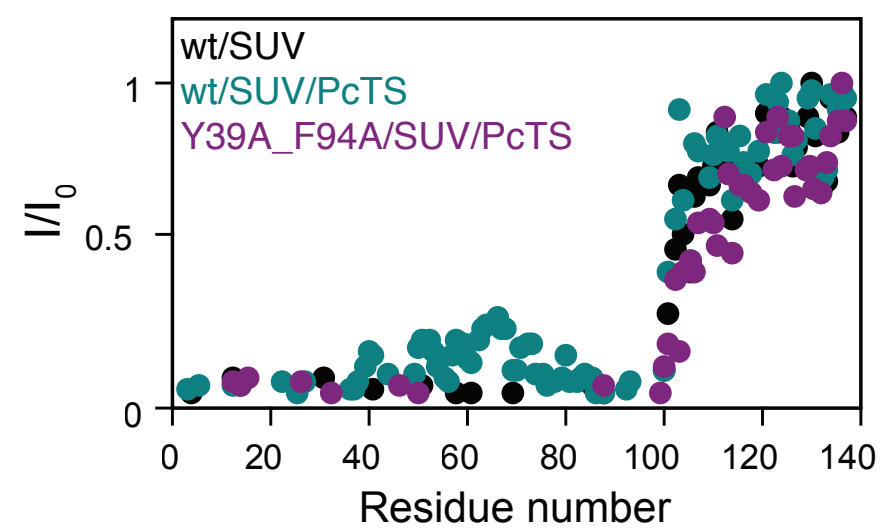

Supplementary Figure 7 | Residue-specific NMR signal intensity ratios obtained from ${ }^{15} \mathrm{~N}-{ }^{1} \mathrm{H}$ HSQC spectra of $30 \mu \mathrm{M}$ of $\alpha$ Syn recorded in the absence $\left(I_{0}\right)$ and presence of $12.5 \mathrm{mM}$ SUVs (I) ( 417:1 lipid to protein molar ratio). Signal intensity ratios for $w t \alpha$ Syn in the absence (black) and in the presence (green) of PcTS are shown. Data for the Y39A/F94A variant of $\alpha$ Syn in the presence of PcTS are shown in purple. The concentration of PcTS was $0.45 \mathrm{mM}$ (15:1 compound to protein molar ratio). Note that when residues are no longer in contact with liposomes, they become "visible" to solution NMR. Thus, even at higher lipid-to-protein ratios PcTS releases the NAC region of $w t \alpha$ Syn from the vesicle surface, but not that of the Y39A/F94A variant.

$0.7 \mathrm{mM}$ PcTS

$0.7 \mathrm{mM}$ PcTS:12.5 mM SUV

$1.4 \mathrm{mM}$ PcTS:12.5 mM SUV
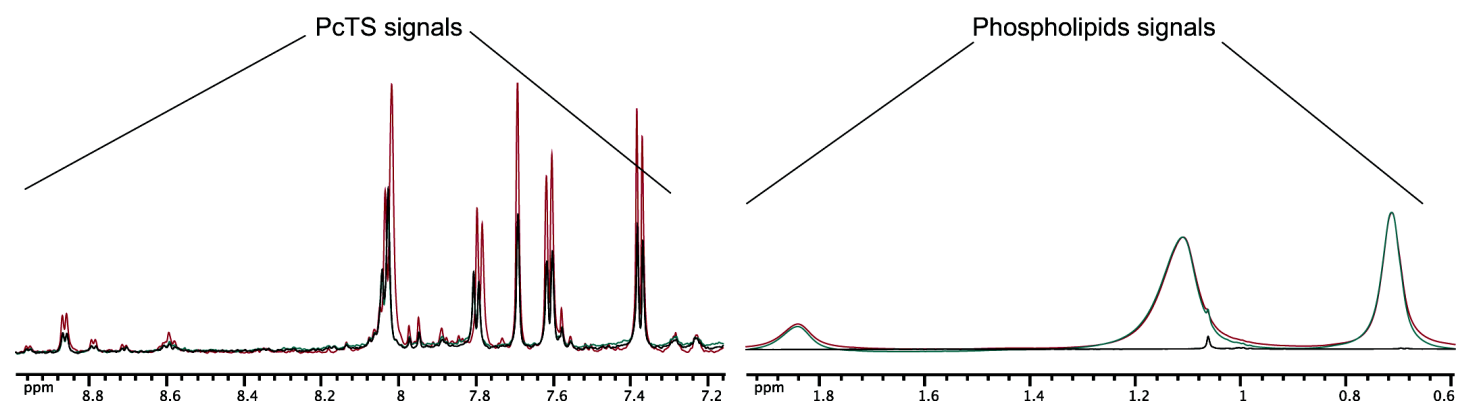

Supplementary Figure 8 | Proton NMR spectra show that PcTS does not interact with SUVs (left panel). Also, increasing amounts of PcTS do not fall out of solution by means of stacking within the concentrations used in the experiments. 


\section{Burial of the Polymorphic Residue 129 in Amyloid Fibrils of}

\section{Prion Stop Mutants}

Lukasz Skora ${ }^{\ddagger 1}$, Luis Fonseca-Ornelas ${ }^{\ddagger}$, Romina V. Hofele ${ }^{\ddagger 1}$, Dietmar Riedel , Karin Giller , Jens Watzlawik ${ }^{\S}$, Walter J. Schulz-Schaeffer ${ }^{\S}$, Henning Urlaub Stefan Becker , and Markus Zweckstetter

From the "Max Planck Institute for Biophysical Chemistry, 37077 Göttingen, the ${ }^{\S}$ Prion and Dementia Research Unit, Department of Neuropathology and the "Department of Clinical Chemistry, University Medical Center Göttingen, 37075 Göttingen, and the German Center for Neurodegenerative Diseases (DZNE), 37077 Göttingen, Germany.

Background: In human prion diseases, the phenotype is modified by a methionine/valine polymorphism at codon 129 .

Results: Prion stop mutants have a conserved amyloid core comprising residue 129.

Conclusion: The polymorphic residue 129 is buried in the amyloid core structure.

Significance: The data support a critical role of the methionine/valine 129 polymorphism in human prion diseases.

Misfolding of the natively alpha-helical prion protein into a beta-sheet rich isoform is related to various human diseases such as Creutzfeldt-Jakob disease and GerstmannSträussler-Scheinker syndrome. In humans, the disease phenotype is modified by a methionine/valine polymorphism at codon 129 of the prion protein gene. Using a 
combination of hydrogen/deuterium exchange coupled to NMR spectroscopy, hydroxyl radical probing detected by mass spectrometry, and site-directed mutagenesis, we demonstrate that stop mutants of the human prion protein have a conserved amyloid core. The 129 residue is deeply buried in the amyloid core structure, and its mutation strongly impacts aggregation. Taken together the data support a critical role of the polymorphic residue 129 of the human prion protein in aggregation and disease.

Misfolding of the natively alpha-helical prion protein $\left(\mathrm{PrP}^{\mathrm{C}}\right)$ into a beta-sheet-rich isoform is related to various human diseases such as Creutzfeldt-Jakob disease and Gerstmann-Sträussler-Scheinker syndrome collectively known as transmissible spongiform encephalopathies (1). The form of disease is determined by two prion types (2), which are a cross species phenomenon (3). Prion types are characterized by differences in stability against denaturing agents, different proteinase K-cleavage sites, and different forms of prion aggregate deposits (3). These differences were attributed to distinct prion conformations (4). In humans, a methionine/valine polymorphism at codon 129 of the prion protein gene contributes in determining disease phenotypes (2). Although both prion types can be formed with each polymorphism at codon 129 , methionine shows a strong association with prion type 1 and valine with prion type $2(5)$. Homozygosity at this position predominates in sporadic Creutzfeldt-Jakob disease, where up to $89 \%$ of patients of large epidemiological studies had either Met/Met or Val/Val at codon 129 (6). It has also been demonstrated that to achieve a more efficient prion transmission in human PrPtransgenic mice models, both the inoculum and the mouse should bear the same amino acid at position 129 (7). 
Although the high-resolution structures of $\operatorname{PrP}^{\mathrm{C}}$ from many species are well described (8), little is known about the structure of $\operatorname{PrP}^{\mathrm{Sc}}$. X-ray fiber diffraction showed that infectious prions have cross-beta structure, confirming that prions can form amyloid (9). Antibody mapping studies suggested that when $\operatorname{PrP}^{\mathrm{C}}$ is converted into $\operatorname{Pr} \mathrm{P}^{\mathrm{Sc}}$, a conformational rearrangement occurs in the region comprising residues 90-176 (10). Mass spectrometric analysis of hydrogen-deuterium exchange of brain-de- rived $\mathrm{PrP}^{\mathrm{Sc}}$ suggested that prion protein conversion involves refolding of the entire region from residues $80-90$ to the $\mathrm{C}$ terminus (11). Electron microscopy of two-dimensional crystals of the 27-30-kDa infectious fragment, PrP27-30, suggested that PrP amyloid fibrils might consist of stacked trimers of left-handed -helices with the core formed by residues $89-140(12-14)$.

Characterization of $\mathrm{PrP}^{\mathrm{Sc}}$ at high resolution is currently not possible. Therefore, a variety of studies have investigated the structure of amyloid fibrils produced in vitro from recombinant prion protein. Mass spectrometry-coupled exchange experiments of amyloid fibrils of human prion protein (humPrP) comprising residues 90-231 indicated strong solvent protection in the region encompassing residues 160-230 (15). In contrast, for mouse $\operatorname{PrP}(23-231)$, residues $24-98$ and 182-212 were protected (16). Hydrogen exchange measurements by mass spectrometry and NMR spectroscopy of mouse $\operatorname{PrP}(89-143)$ bearing a P101L mutation identified a high level of protection from solvent exchange for residues 102-109 and 117-136 (17). In addition, molecular dynamics simulations provided models for the conformation of PrP in prions (18).

Hereditary prion diseases include C-terminally truncated variants of the prion protein, 
Y145X, Q160X, Y226X, and Q227X, where $X$ indicates the truncation site (see Fig. 1A). Although a single experiment to transmit human 145stop mutant prions to mice failed (19), the stop mutants provide a particularly valuable model for studying misfolding of prion protein $(20,21)$ as they: (i) lack the normally present glycosylphosphatidylinositol anchor and are secreted into the intercellular medium, which appears to favor fibrillization and aggregation (21, 22); (ii) show in vivo prion deposits in the form of amyloidogenic plaques or plaque-like aggregates, like other prion diseases (23); and (iii) aggregate under nondenaturing conditions in vitro (20, 22). Previously, we showed that the -sheet content is highly similar in amyloid fibrils of the Y145X and Q160X prion stop mutants (22), and solid-state NMR spectroscopy revealed extended beta-sheet conformation in the 112-140 region (24-26). Here we investigated the importance of the polymorphic residue 129 for the formation and structure of amyloid fibrils of human prion stop mutants. Using a combination of hydrogen/deuterium (H/D) exchange coupled to NMR spectroscopy, hydroxyl radical probing detected by mass spectrometry, and site-directed mutagenesis, we demonstrate that the valine/methionine residue 129 is deeply buried in the amyloid core of the stop mutants, supporting its critical role in aggregation and disease.

\section{EXPERIMENTAL PROCEDURES}

Protein Expression and Purification-Plasmids encoding Q160X and N174X were expressed in Escherichia coli BL21(DE3) by using M9 minimal medium with [ ${ }^{15}$ $\mathrm{N} \mathrm{NH}_{4} \mathrm{Cl}(1 \mathrm{~g} /$ liter $)$ as the only nitrogen source and as required $\left[{ }^{13} \mathrm{C}_{6}\right]$ glucose $(4$ g/liter), to obtain uniformly ${ }^{15} \mathrm{~N}$ - or ${ }^{13} \mathrm{C},{ }^{15} \mathrm{~N}$ - labeled protein, and purified according to Ref. 27. Variants of humPrP(108-143) with purity exceeding 95\% were obtained from EZBiolab. 
Aggregation of $Q 160 X$ and N174X into Amyloid Fibrils-Aggregation of Q160X and $\mathrm{N} 174 X$ was started by transferring protein solutions into a phosphate buffer (50 mM potassium phosphate, $\mathrm{pH}$ 6.5) (22). The reaction was carried at $25{ }^{\circ} \mathrm{C}$ without agitation, and fibril formation was monitored using a thioflavin $\mathrm{T}$ fluorescence assay.

Aggregation of humPrP(108 -143) — Variants of lyophilized humPrP(108-143) were dissolved at a concentration of $0.35 \mathrm{~mm}$ with ice-cold $25 \mathrm{~mm}$ Tris buffer, $\mathrm{pH} 7.5$, $0.02 \%$ sodium azide and dialyzed overnight against $500 \mathrm{ml}$ of $25 \mathrm{~mm}$ Tris, $\mathrm{pH} 7.5$, $0.02 \%$ sodium azide at $4{ }^{\circ} \mathrm{C}$ in a $500-1000$-Da molecular mass cutoff dialysis membrane. The peptide was filtered through a 0.22- $\mathrm{m}$ Millipore filter. Concentration was adjusted to $0.15 \mathrm{~mm}$ in a $500 \mu \mathrm{l}$ final volume, and the solution was incubated at $12{ }^{\circ} \mathrm{C}$ in low binding protein $1.5-\mathrm{ml}$ Eppendorf tubes. After different incubation times, a $20 \mu \mathrm{l}$ aliquot of the sample was mixed with $1 \mathrm{ml}$ of the thioflavin $\mathrm{T}$ assay solution ( 0.1 mм thioflavin $\mathrm{T}$ in $\left.100 \mathrm{~mm} \mathrm{NaH}_{2} \mathrm{PO}_{4}, 140 \mathrm{~mm} \mathrm{NaCl}, \mathrm{pH} 8.5\right)$, incubated 5 min at room temperature and transferred to a $10 \mathrm{~mm}$ cuvette. The fluorescence emission was measured between 460 and $600 \mathrm{~nm}$ on a Varian Cary Eclipse fluorescence spectrophotometer (Agilent Technologies) with excitation at $442 \mathrm{~nm}$ at $20^{\circ} \mathrm{C}$.

Quantification of Aggregate Formation by UV Absorbance- To have a second measure for aggregate formation of different variants of humPrP(108 -143), aggregates were collected after 2.7 and $24 \mathrm{~h}$ of incubation by centrifugation at 16,000 $g$ for 30 min at $4{ }^{\circ} \mathrm{C}$. Subsequently, the pellet was resuspended in $100 \mu \mathrm{l}$ of $25 \mathrm{~mm}$ Tris $\mathrm{HCl}, \mathrm{pH} 7.5$, and diluted in a 1:5 ratio in $7.5 \mathrm{~m}$ guanidine hydrochloride, $25 \mathrm{~mm}$ $\mathrm{NaH}_{2} \mathrm{PO}_{4}, \mathrm{pH}$ 6.5. Peptide concentrations were determined by UV absorption at 280 $\mathrm{nm}$. 
Electron Microscopy (EM) - The sample was bound to carbon-coated grids and stained with $1 \%$ uranyl acetate. Pictures were taken at 72,000 magnification at a CM 120 with a 20482048 pixel TemCam (Tietz) in spotscan mode.

NMR-detected H/D Exchange-Fibrils were collected by centrifugation (10,000 $\mathrm{g}$ for 5 min) and washed 3-4 times with $50 \mathrm{~mm}$ phosphate buffer ( $\mathrm{pH} 6.5)$ to remove residual monomeric protein or low molecular weight oligomers. To initiate exchange, the pellet was resuspended in $\mathrm{D}_{2} \mathrm{O}$ buffer $\left(0.1 \%\right.$ formic acid in $\mathrm{D}_{2} \mathrm{O}$, pD 2.5$)$ and incubated at $4{ }^{\circ} \mathrm{C}$ for 3 and 7 days. The samples were then immediately frozen in liquid nitrogen and lyophilized. For the NMR experiments, lyophilized proteins were resolubilized in ice-cold solution of 2 м guanidinium thiocyanate in $50 \% \mathrm{H}_{2} \mathrm{O}, 50 \%$ $\mathrm{D}_{2} \mathrm{O}, \mathrm{pD} 2.5$, and a series of ${ }^{1} \mathrm{H},{ }^{15} \mathrm{~N}$ heteronuclear single quantum coherence (HSQC) spectra was recorded over a period of $24 \mathrm{~h}$ to follow the back-exchange process (28). NMR spectra were acquired at $278 \mathrm{~K}$ on a Bruker Avance $900 \mathrm{MHz}$ spectrometer equipped with a 5-mm triple resonance cryogenic probe head. Experiment time was $23 \mathrm{~min}$, and the dead time for sample preparation and acquisition setup was $15 \mathrm{~min}$. NMR data were processed and analyzed using NMRPipe (29). For non resolvable residues within the octarepeat region, protection levels were calculated using averaged intensities. Signals originating from residues Gly-30, Trp-31, Gly-35, Arg-37, Tyr-38, Tyr-49, Gly-53, Gly-55, Trp-57, Met-109, Lys-110, Met-112, Leu-125, Ile138, and Tyr-145 were overlapping in the denaturing condition. As signal overlap might distort the analysis and lead to false protection factors, these residues were not included into the analysis. Exchange curves were fitted to Equation 1 using IgorPro 5.01

$I / I_{0}=A 1+A 2 \times \exp (A 3 \times t)$ 
where $I_{0}$ is the signal intensity observed in the first HSQC spectrum after dissolution, and $A 1-A 3$ are fitting variables.

NMR Resonance Assignment - Sequential backbone assignment of humPrP mutants in fibril dissolving buffer was obtained from three-dimensional HACANNH, HNCACB, and HNN experiments $(30,31)$ recorded at $278 \mathrm{~K}$ on a Bruker Avance spectrometer operating at $900 \mathrm{MHz}$ and equipped with a 5-mm triple resonance cryo probe. The samples used for resonance assignment contained typically $0.4 \mathrm{~mm}{ }^{13} \mathrm{C},{ }^{15} \mathrm{~N}$-labeled variant humPrP in 2 m guanidinium thiocyanate, $90 \% \mathrm{H}_{2} \mathrm{O}, 10 \% \mathrm{D}_{2} \mathrm{O}, \mathrm{pH} 2.5$. Due to severe signal overlap in the octarepeat region, only 89 resonances for 123 non-proline residues of human humPrP(23-159) were observed in two-dimensional ${ }^{1} \mathrm{H},{ }^{15} \mathrm{~N}$ HSQC spectra of the denatured monomer. 64 of these resonances could be assigned unambiguously, whereas the rest could only be located to the octarepeat region.

Measurement of Diffusion Coefficients - The molecular weight of the amyloid fibrils was estimated using NMR pulsed field gradient methods experiments (32). $10 \mathrm{mg}$ of unlabeled fibrils were placed in a rotor, and diffusion coefficients were measured using the LED pulse sequence with bipolar gradients (33). Spectra were recorded on a Bruker Avance 900-MHz spectrometer equipped with a 4-mm high-resolution magic angle spinning probe. The sample was spun at $7.2 \mathrm{kHz}$, total diffusion time was $D 100$ ms, and gradient pulse length $d 3 \mathrm{~ms}$. Sine-shaped gradients were used, and their strength was incremented in 16 steps from $g$ 0.07-0.48 $\mathrm{G} / \mathrm{mm}$. The diffusion coefficients were calculated by fitting the intensity decay curves (see Fig. 1C) with the equation

$$
I / I_{0}=\exp \left[-D(\gamma d g)^{2}(D-d / 3-t / 2)\right]
$$


where is the gyromagnetic ratio of the ${ }^{1} \mathrm{H}$ nucleus, and $t 200 \mathrm{~s}$ is the delay for gradient switching. For molecular weight estimation, 1,4-dioxane (88 Da) and bovine serum albumin $(66 \mathrm{kDa})$ were used as internal and external references.

Mass Spectrometry $(M S)$ - humPrP(108 -143) was probed in the monomeric and fibrillar state by chemically generated hydroxyl radicals, as described in Ref. 34. 701 of $50 \mathrm{~m}$ sample were added to an Eppendorf tube containing 101 of $13 \mathrm{~mm}$ Fe-EDTA (Sigma-Aldrich and Merck, respectively) solution and 101 of 0.2 m sodium ascorbate (Sigma-Aldrich) solution, both in $50 \mathrm{~mm} \mathrm{Na} \mathrm{HPO}_{4}, \mathrm{pH} 6.5$ buffer. For control purposes, a $17 \mu \mathrm{l}$ aliquot was transferred to $40 \mu \mathrm{l}$ of quenching buffer $(2 \mathrm{~m}$ Tris, $\mathrm{pH}$ 7.2, Prolabo). Finally, $10 \mu \mathrm{l}$ of $3 \% \mathrm{v} / \mathrm{v} \mathrm{H}_{2} \mathrm{O}_{2}$ (Sigma-Aldrich) were added to the Fenton mixture to start the reaction. At $10,60,150$, and $300 \mathrm{~s}, 17 \mu$ l aliquots were removed and transferred to new Eppendorf tubes containing 401 of quenching buffer.

To disassemble the probed peptides prior to further analysis, samples were dissolved in 2001 of acetonitrile. The total volume was reduced to $20 \mu 1$. Subsequently, $150 \mu 1$ of $100 \mathrm{~mm}$ Tris, $10 \mathrm{~mm} \mathrm{CaCl}_{2}$ buffer and $0.5 \mathrm{~g}$ of chymotrypsin (Roche Applied Science) were added, and digestion was allowed to proceed overnight at $25{ }^{\circ} \mathrm{C}$. The digested samples were desalted with C8 (3M) STAGE tips (35).

Nano-liquid Chromatography Separation and MS Analysis- For LC-MS/MS analyses, samples were dissolved in $30 \mu \mathrm{l}$ of sample solvent $(5 \% \mathrm{v} / \mathrm{v}$ acetonitrile, $1 \%$ $\mathrm{v} / \mathrm{v}$ formic acid). $5 \mu$ l were injected onto a nano-liquid chromatography system (Agilent 1100 series, Agilent Technologies) including an 2-cm- long, $150 \mu \mathrm{m}$ inner diameter C18 trapping column in-line with an 15-cm-long, $75 \mu \mathrm{m}$ inner diameter C18 analytical column (both packed in-house, C18 AQ $120 \AA 5$ m, Dr. Maisch GmbH, 
Ammerbuch, Germany). Peptides were loaded on the trapping column at a flow rate of $10 \mathrm{l} / \mathrm{min}$ in buffer $\mathrm{A}\left(0.1 \%\right.$ formic acid in $\left.\mathrm{H}_{2} \mathrm{O}, \mathrm{v} / \mathrm{v}\right)$ and subsequently eluted and separated on the analytical column with a gradient of $7.5-37.5 \%$ buffer B $(95 \%$ acetonitrile, $0.1 \%$ formic acid in $\mathrm{H}_{2} \mathrm{O}$, v/v) with an elution time of $37 \mathrm{~min}$ and a flow rate of $300 \mathrm{nl} / \mathrm{min}$.

Online electrospray mass ionization-MS was performed with an LTQ-Orbitrap Velos instrument (Thermo Scientific), operated in data-dependent mode using a TOP10 method. MS scans were recorded in the $m / z$ range of 350-1600. The 10 most intense ions were selected for subsequent MS/MS. Both precursor ions as well as fragment ions were scanned in the Orbitrap. Fragment ions were generated by higher energy collision dissociation activation (normalized collision energy 40) and recorded from $m / z 100$. As precursor ions as well as fragment ions were scanned in the Orbitrap, the resulting spectra were measured with high accuracy $(5 \mathrm{ppm})$ both in the MS and in the MS/MS level.

Determination of Oxidized Sites - Data analysis was per- formed using MaxQuant 1.1.1.14 (36). Database search was performed against humPrP(108-143) with Andromeda (37) considering a total of 14 variable modifications: hydroxylation (16 Da) in His, Val, Met, Leu, Tyr, Arg, Pro, Ile, Phe; dioxidation (32 Da) of Met, Tyr, Phe; deguanidination (43 Da) in Arg; and ring opening (22) in His. The oxidation levels at any given time point were calculated on a per-residue basis. By making use of extracted ion chromatograms, obtained with Xcalibur (10 ppm tolerance), the ratios of oxidized to nonoxidized peptides in which a specific residue is involved were determined according to Ref. 38 and as illustrated below for Met-112 
where $\mathrm{O}$ indicates the oxidized residue. Because ionization efficiencies of modified and unmodified species are similar, but not identical, the results need be interpreted in a relative context (i.e. fibrillar versus monomeric states). For quantification of peptides that contain several modifications in different combinations that lead to the same $\mathrm{m} / \mathrm{z}$ value (termed isobaric oxidized peptides), we made use of the fact that these elute at different retention times from the LC into the mass spectrometer (ranging from 1 to 9 min with respect to the non oxidized peptide). To the same purpose, only the most abundant charge state of each peptide was considered.

Error Calculation-Two independently prepared fibril samples and two monomeric samples were measured and used for error calculation, and the error informed corresponds to the S.E. It should be noted that the sum of the intensities corresponding to different combinations of oxidized residues (the case for most methionines) lead to higher uncertainties, which stresses the need for the results to be interpreted in a relative context.

\section{RESULTS}

Size Estimation of humPrP(23-159) Fibrils - To characterize the molecular weight of humPrP(23-159) fibrils (Fig. 1B), we have used pulsed-field gradient methods (32) combined with high-resolution magic angle spinning (Fig. 1C). High-resolution magic angle spinning averages out NMR signal broadening caused by inhomogeneity in magnetic field anisotropy and thus allows detection of flexible regions in aggregates, whereas NMR-based pulsed field gradient methods provide an estimate of the 
diffusion coefficient of aggregates. To take into account the nonspherical shape of fibrils and contribution from rotational diffusion, the equations described by Perrin (39) were applied to calculate the corresponding diffusion constant of a spherical molecule of the same molecular weight. For this calculation, the average diameter and length of the fibrils were estimated from EM micrographs as 15 and $500 \mathrm{~nm}$, respectively. Using the Stokes-Einstein relation, the molecular weight of humPrP(23159) fibrils was estimated to be in the order of $4 \mathrm{MDa}$.

Solvent Protection in the Fibrillar State-The solvent accessibilities of single residues in amyloid fibrils (Fig. 2) of the hereditary stop mutant Q160X were probed using NMR-detected H/D exchange (40). To allow detection by liquid-state NMR and at the same time preserve the H/D exchange pattern, fibrils that had been exposed to $\mathrm{H} / \mathrm{D}$ exchange were rapidly converted to monomers in a $2 \mathrm{~m}$ solution of guanidinium thiocyanate in $50 \% \mathrm{H}_{2} \mathrm{O}, 50 \% \mathrm{D}_{2} \mathrm{O}(28)$. Back-exchange was then monitored in the denatured state by a series of two-dimensional ${ }^{1} \mathrm{H},{ }^{15} \mathrm{~N}$ HSQC spectra (Fig. 2). Neglecting back-exchange before and during the first HSQC after dissolution, the intensity ratio is 1.0 for an amide proton, for which the protection level in the fibril is $50 \%$, and reaches a minimum of 0.5 for an amide proton with a $100 \%$ protection level in the fibrillar state.

To allow a residue-specific analysis of the H/D exchange, an assignment of the denatured protein in the dissolving buffer was required. Due to severe signal overlap in the octarepeat region, 89 distinct resonances for 123 non-proline residues of human humPrP(23-159) were observed in two-dimensional ${ }^{1} \mathrm{H},{ }^{15} \mathrm{~N}$ HSQC spectra of the denatured monomer. Sequence- specific backbone assignment was obtained for 64 of these resonances from a set of standard three-dimensional HACANNH, HNCACB, 
and HNN experiments $(30,31)$. The rest could only be located to the octarepeat region. Based on the sequence- specific resonance assignment, a solvent protection map was constructed that revealed back-exchange ratios of more than 1.2 for the segments $23-108$ and 143-173 (Fig. 3A). In the central domain comprising residues 109-142, two regions could be distinguished: residues 109-120 and 140-142 with intermediate amide proton back-exchange ratios (exchange ratios of 1.0-1.2) and residues $121-139$ with high protection values (70\%). A very similar protection map was obtained after 3 instead of 7 days of H/D exchange (Fig. $3 A$ ).

Methionine 129 Is Strongly Protected from Solvent Exchange-Comparison of H/D exchange profiles for individual residues (Figs. $2 B$ and $3 A$ ) provides further insight. Firstly, within the region 109-140, Gly-119 and Ala-120 showed the most pronounced increase in signal intensity during the back-exchange process, suggesting that a turn may be formed by these residues. Secondly, the striking change in the exchange profiles from Ile-139 to His-140 points to a well-defined boundary of the fibrillar core. Thirdly, residues 144-156 that form helix1 in the native structure of the human prion protein (8) showed high back-exchange ratios, comparable with the flexible N-terminal domain and suggesting that the helix1 region is highly solventaccessible in amyloid fibrils of humPrP(23-159). Fourth, the polymorphic residue Met-129 is strongly protected from solvent exchange. 

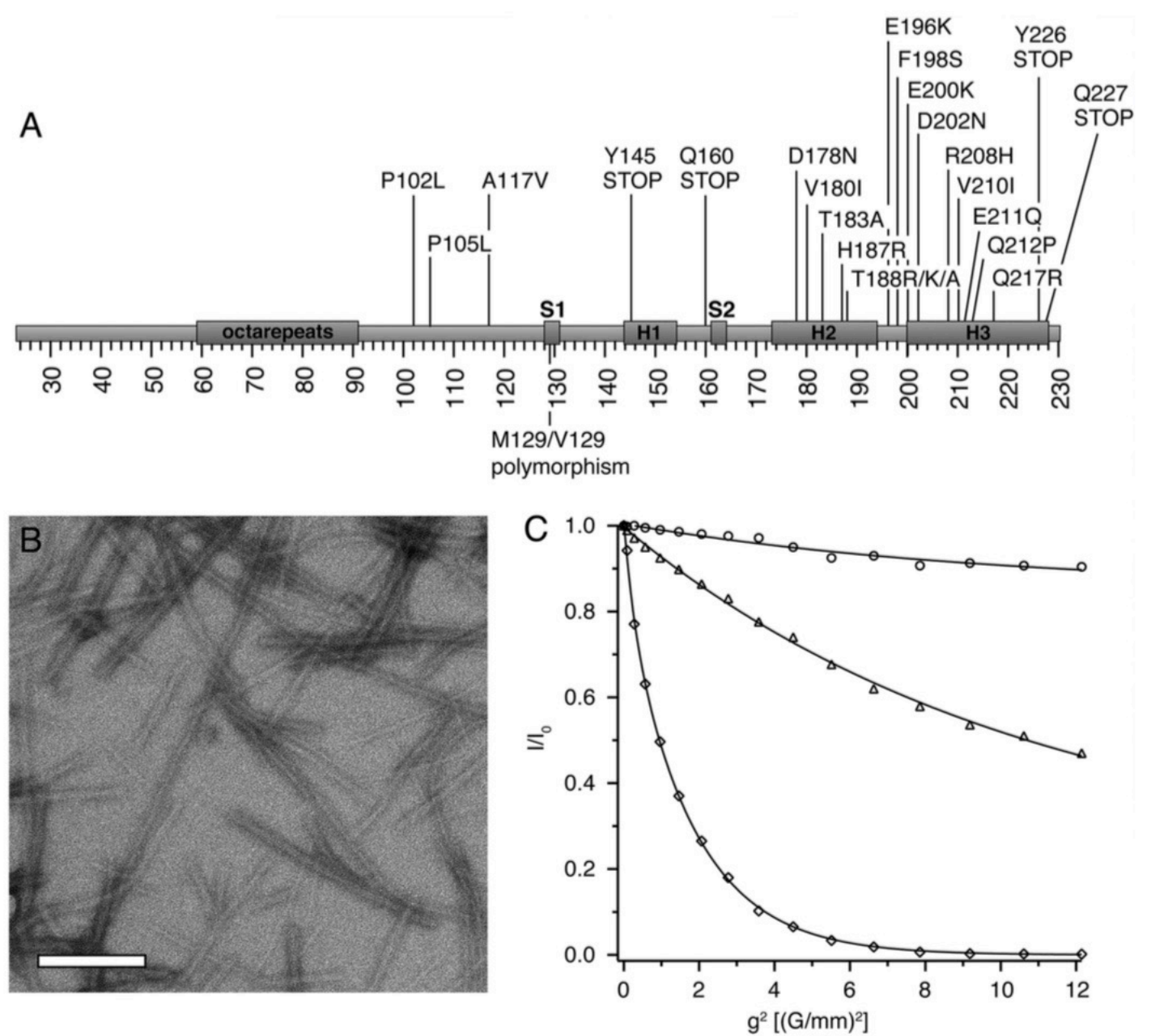

FIGURE 1. Amyloid fibrils of humPrP(23-159). $A$, schematic representation of native secondary structure elements and point mutations found in the human prion protein. $B$, electron micrographs of mature fibrils. The white bar indicates $200 \mathrm{~nm}$. $C$, decay of NMR signal intensity under pulsed field gradients recorded for humPrP(23159) fibrils (circles), bovine serum albumin (triangles), and 1,4-dioxane (diamonds). The measured diffusion coefficient of fibrils of humPrP(23-159) was $2.610^{11} \mathrm{~m}^{2} / \mathrm{s}$. 

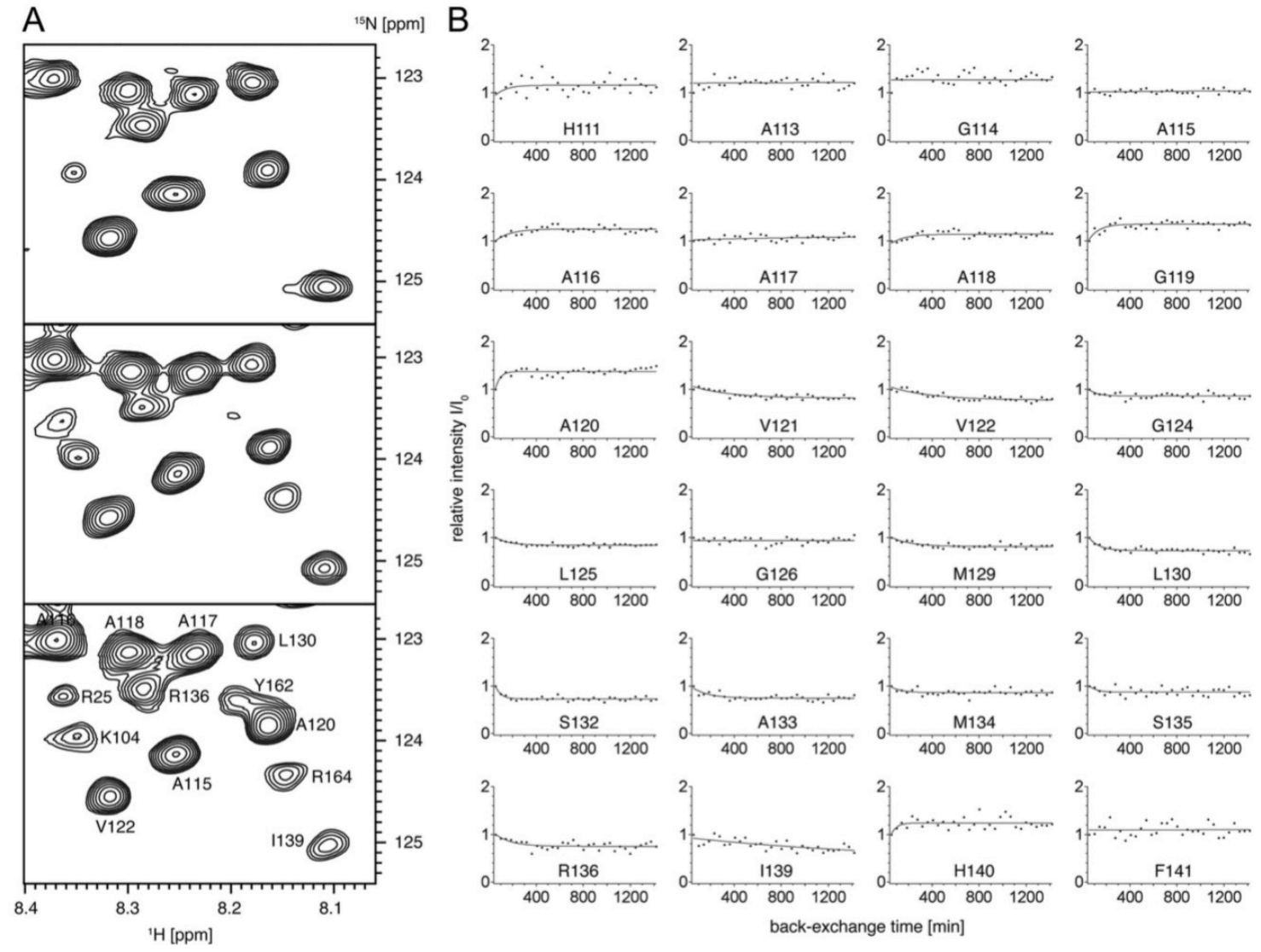

FIGURE 2. Solvent protection in amyloid fibrils of the Q160X stop mutant. $A$, excerpts from two-dimensional ${ }^{1} \mathrm{H},{ }^{15} \mathrm{~N}$ correlation spectra showing changes in signal intensities during the back-exchange process. The spectra were taken 1 (top), 6 (middle), and $24 \mathrm{~h}$ (bottom) after the dissolution of fibrils. Sequence- specific assignments are indicated. $B$, changes in signal intensities over time of individual residues in the region 111-141 due to back-exchange in the denatured monomeric state. Only data for residues that do not overlap in the two-dimensional ${ }^{1} \mathrm{H},{ }^{15} \mathrm{~N}$ HSQCs are shown.

A Common Amyloid Core of Human Prion Stop Mutants - To probe the structural consequences of residues ${ }^{160} \mathrm{QVYY}^{163}$, which form beta-strand 2 in the native $\operatorname{PrP}$ structure, we performed H/D exchange on amyloid fibrils of the designed stop mutant $\mathrm{N} 174 X$. The solvent protection map of $\mathrm{N} 174 X$ was very similar to the exchange profile of the Q160X stop mutant (Fig. 3B). The high solvent accessibility of residues 
$160-170$ suggested that this region is not part of the fibrillar core in the $\mathrm{N} 174 X$ stop mutant. In longer peptides, however, more extensive structure could be present in this sequence stretch. The combined H/D exchange data indicate that the hydrogenbonded core of amyloid fibrils of Q160X and N174 $X$ stop mutants of the human prion protein comprises residues 121-139, flanked by residues 109-120 and 140-142.

The Side Chain of the Polymorphic Residue 129 Is Buried in the Amyloid CoreNext, we investigated the organization of single side chains in the amyloid structure of prion stop mutants using hydroxyl radical probing (Fig. 4). Hydroxyl radicals covalently modify side chains of solvent-accessible amino acids. The modifications induce a mass shift that can be detected by mass spectrometry (41). Measurements were performed for the peptide humPrP(108-143) that covers the fibrillar core of the stop mutants (Fig. 3) (24-26) and for which amyloid fibrils are morphologically similar to those of the stop mutants (Fig. 5). When compared with the stop mutants, the humPrP(108-143) peptide has two important advantages: (i) peptides obtained by solid-phase synthesis and stored as lyophilized powder are less prone to oxidation of methionine residues, a problem previously reported for recombinant prion protein (42); and (ii) the disordered N-terminal tail comprising residues 23-107 does not modulate the rate of aggregation.

Hydroxyl radical probing was applied to humPrP $(108-143)$ in both the disordered monomer and the fibrillar state (Fig. 4 and supplemental Fig. S1). Oxidative modifications were observed for residues Met-109, Met-112, Tyr-128, Met-129, Leu130, Met-134, Arg-136, Pro-137, Ile-138, Ile-139, His-140, and Phe-141 (Fig. 4 and supplemental Fig. S2). Other amino acid residues were not modified because of their lower reactivity or solvent accessibility. Strikingly, a pronounced reduction in 
oxidation was observed for the side chains of Tyr-128, Met-129, and Leu-130 in the fibrillar state (Fig. 4B). In addition, small differences in oxidation levels were present for Ile-138 and Phe-141. No changes in oxidation were observed for the side chains of Met-109, Met-112, Met-134, Arg-136, Pro-137, Ile-139, and His-140 despite the fact that the backbone amide protons of several of these residues are part of a fibrillar beta-strand (24).
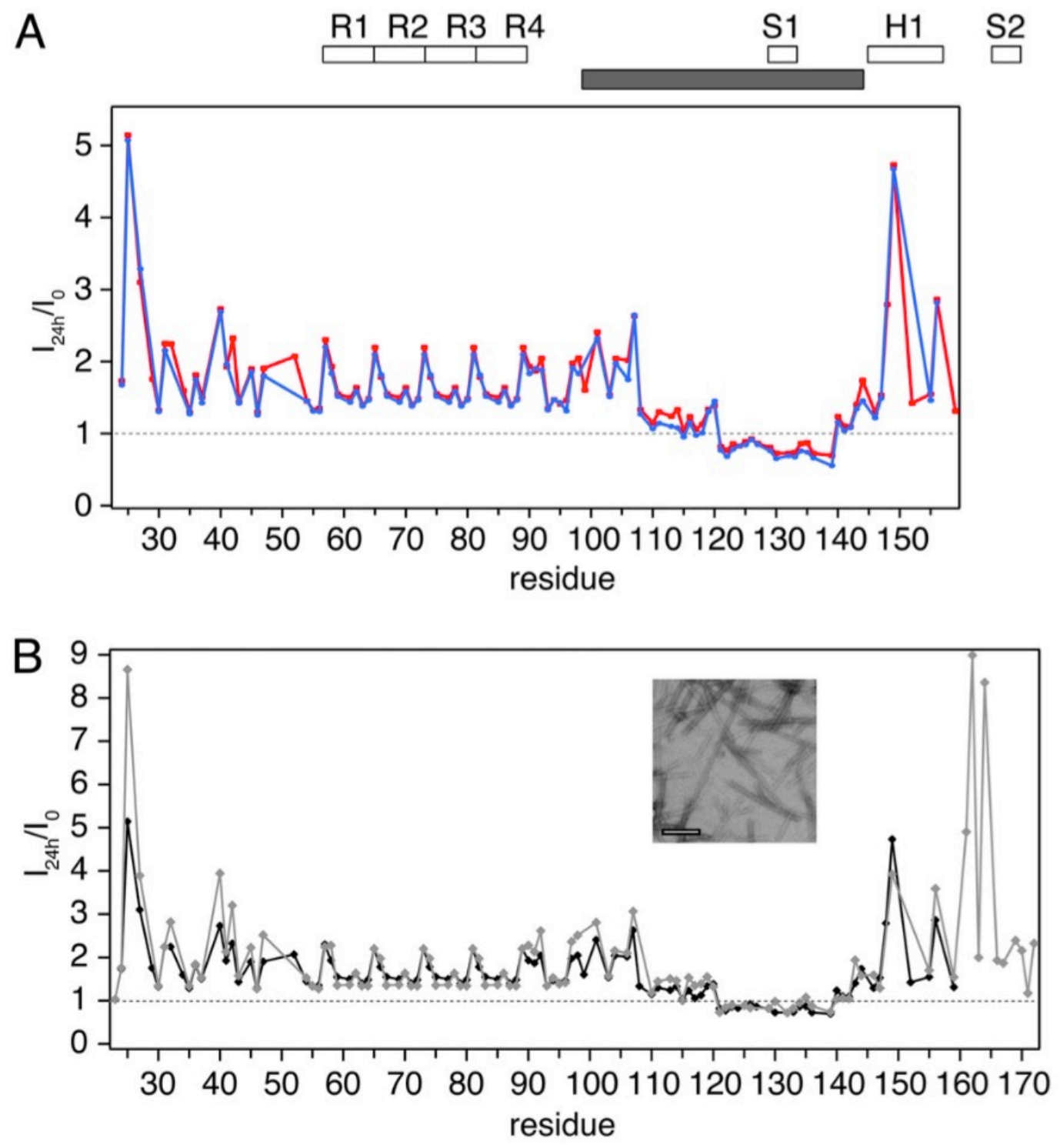

FIGURE 3. A conserved solvent-protected core in amyloid fibrils of stop mutants 
of the human prion protein. $A$, comparison of the $\mathrm{H} / \mathrm{D}$ protection maps of humPrP(23-159) fibrils exchanged in $\mathrm{D}_{2} \mathrm{O}$ for 3 days (blue) and 7 days (red). $R 1-R 4$ are the octarepeats. Above part of the domain organization of PrpC is shown (strands $\mathrm{S} 1$ and $\mathrm{S} 2$; helix $\mathrm{H} 1$; repeats $\mathrm{R} 1-\mathrm{R} 4) . B, \mathrm{H} / \mathrm{D}$ protection maps of humPrP(23-159) (black) and humPrP(23-173) (gray) fibrils exchanged in $\mathrm{D}_{2} \mathrm{O}$ for 7 days. In $A$ and $B$, $I_{24 \mathrm{~h}}$ is the signal intensity after $24 \mathrm{~h}$ of back-exchange relative to the signal intensity $I_{0}$ observed in the first HSQC spectrum after dissolution. $I_{24 \mathrm{~h}} / I_{0} 1.0$ indicates strong protection from exchange. The location of the proteinase K-resistant core is shown by a gray bar. Only data for nonoverlapping residues are shown. The inset shows an electron micrograph of humPrP(23-159) fibrils.

Position 129 Is Critical for Aggregation of Human Prion Stop Mutants-The burial of the side chain of Met-129 in the fibrillar core suggests an important role of this residue for aggregation of the prion protein. To test this hypothesis, we introduced point mutations into humPrP(108-143). Removal of the hydrophobic side chain by the substitutions M129N and M129D inhibited the aggregation of humPrP(108-143) (Fig. 5). In contrast, substitution of Met-109 by lysine did not have any effect, consistent with the location of Met-109 outside of the solvent-protected region (Fig. 3, Table 1). Importantly, substitution of Met-129 with valine reduced the lag time of fibril formation. Moreover, when mixing humPrP(108-143) with the Val-129 variant at a ratio of 1:3, the lag phase of the aggregation was in between that of the two pure peptides, whereas at an equal ratio of the two variants, aggregation was retarded when compared with the Met-129 variant alone (Fig. 5A). Thus, a 1:1 mixture of Met-129 and Val-129 humPrP(108-143) aggregates slower than the pure peptides at the same total con- centration, suggesting that the two variants are not as active in the formation of aggregation nuclei as the pure sequences. 
The importance of the 129 position for prion aggregation was further supported by seeding experiments. After 6 h of aggregation, Met-129 humPrP(108-143) amyloid fibrils were fragmented and homogenized with three 5-min sonication cycles. Three different volume/volume percentages $(0.1,1$, and $5 \%)$ were used as seeds on fresh preparations of monomeric Met- 129, Asn-129, and Asp-129 peptides. Regardless of the seed concentration, there was no seeding effect on either Asn-129 or Asp-129 peptides, whereas the Met-129 seed on Met-129 fresh monomer completely removed the nucleation phase (Fig. 5D).

\section{DISCUSSION}

Biochemical analyses of aggregated prion protein in patients with a stop codon mutation suffering from a prion disease have shown that the protein resulting from the mutated allele forms the aggregates $(23,43,44)$. However, a single experiment to transmit human 145 stop mutant prions to New Zealand White mice failed (19).

Using H/D exchange coupled to NMR spectroscopy, we showed that the Q160X and N174X stop mutants of the human prion protein have a common solvent-protected core (Fig. 3) that starts at residue 109 and ends at residue 142. Residues ${ }^{160}$ QVYY ${ }^{163}$, which form beta-strand 2 in the native PrP structure, are not part of the fibrillar core of these stop mutants. In addition, residues $144-156$ that form helix 1 in the native structure (8) remain highly solvent-accessible in amyloid fibrils of humPrP(23-159). In vitro aggregated amyloid fibrils of Q160X and Y145X are morphologically similar according to electron microscopy $(20,22)$ and have similar proteinase $\mathrm{K}$ cleavage sites (22). Moreover, solid-state NMR measurements of Y145X located the betasheet-rich amyloid core to residues 112-140 (24). Taken together the data suggest that their amyloid fibrils share a common structure starting at residue 109-112 and ending 
at residue 140-142. The identified fibril core is consistent with a major PrP fragment of $7 \mathrm{kDa}$ that was detected in amyloid fibrils purified from the 145stop mutant (43), from the 227stop mutant (44), and from Gerstmann-Sträussler-Scheinker brains and spans residues $81-82$ to $144-153$ (45). Moreover, the fibrillar core represents the most conserved sequence element in mammalian and non mammalian prion proteins (46) and encompasses the peptide fragment 112-119 that is essential for the replication of the infectious agent in tissue culture (47).

Recently, some of us determined the backbone fold of amyloid fibrils of the Y145X stop mutant by application of the CS-Rosetta structure calculation program to experimental solid- state NMR chemical shifts $(24,48)$. The calculations revealed a left-handed beta-helix formed by three beta-strands. The Met-129 side chain is located in the beginning of the third beta-strand and points inward to the hydrophobic core of the left-handed beta-helix. The structural model is well supported by the solvent accessibility of individual side chains obtained by mass spectrometry. Met-129 shows a pronounced reduction of solvent accessibility in the amyloid state (Fig. 4), in line with the structural model proposed for the Y145X stop mutant (48).

Besides Met-129, the side chains of Tyr-128 and Leu-130 are solvent-protected in the amyloid state according to mass spectrometry (Fig. 4). In the beta-helix model of the Y145X stop mutant, the two residues belong, together with Met-129, to a single betastrand formed by ${ }^{128}$ YMLGSAMSR $^{136}(48)$. The experimental solvent accessibilities of the ${ }^{128}$ YMLGSAMSR ${ }^{136}$ beta-strand suggest that both sides of this beta-strand are protected. The side harboring the Met-129 side chain forms the hydrophobic core of the beta-helix. In contrast, the side harboring Tyr-128 and Leu-130 is not protected in a single filament. A possible explanation for the solvent protection of the Tyr-128 and 
Leu-130 side chains in light of the beta-helix model could be the pairing of two or more filaments, a hypothesis that would be supported by the dimensions of amyloid fibrils of the prion stop mutants observed by electron microscopy (22).
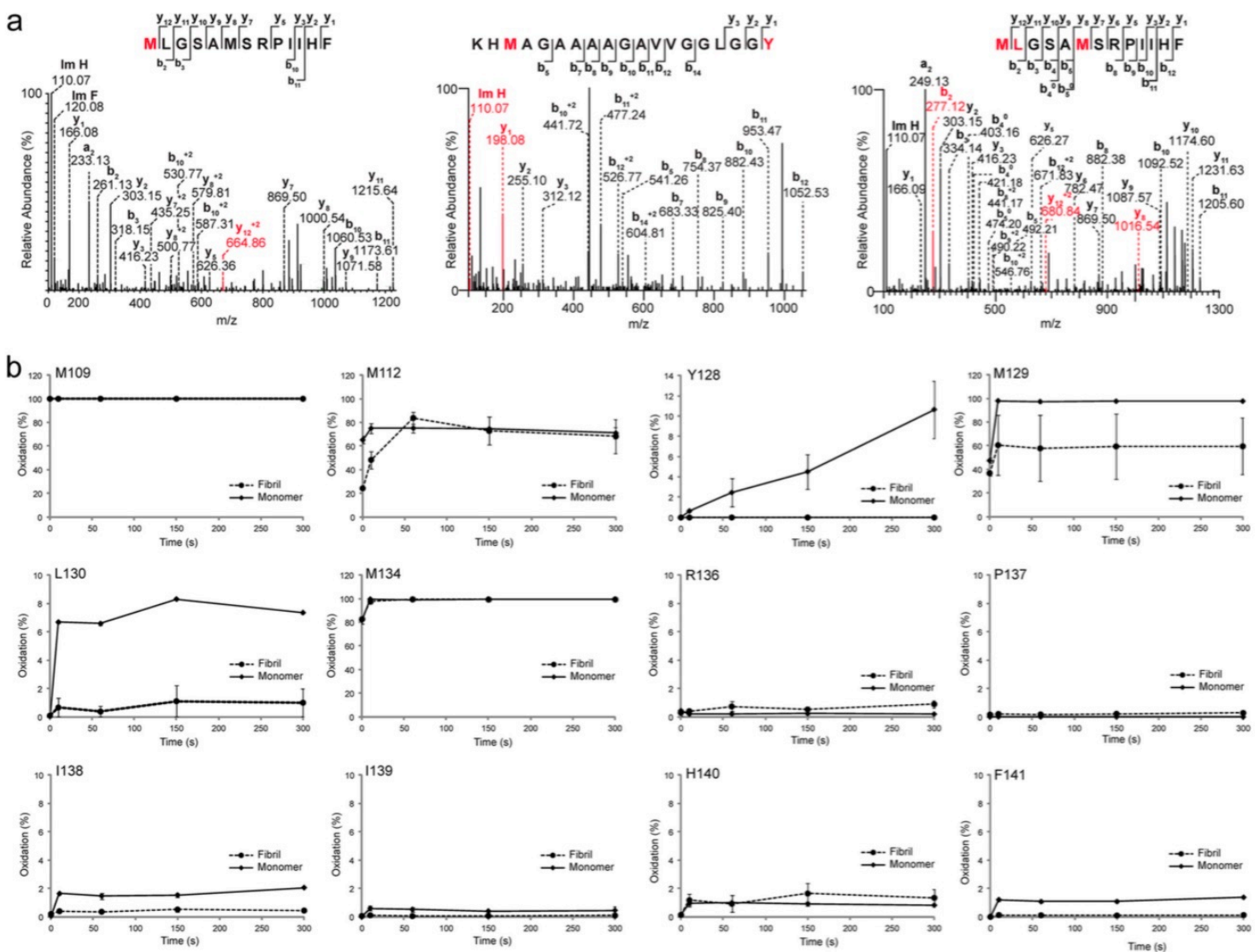

FIGURE 4. Burial of the Met-129 side chain in amyloid fibrils of humPrP(108 -

143). $A$, tandem MS spectra of oxidized humPrP $(108-143)$. The oxidized amino acid and its corresponding fragment ions containing the modification are depicted in red, and $b$ and $y$ ions indicate fragmentation of the peptide bond conserving the charge to the $\mathrm{N}$ or $\mathrm{C}$ terminus, respectively. In the peptide MLGSAMSRPIIHF (129 -141), residues Met-129, Leu-130, and Met-134 are oxidized. $B$, time-course modifications of single residues for the fibrillar (dashed line) and monomeric state (solid line). Error bars correspond to S.E. obtained from independent measurements on two fibrillar and two monomeric samples. 
In humans, the disease phenotype is modified by a methionine/valine polymorphism at codon 129 of the prion protein gene in addition to the prion type. Increasing evidence suggests that changes between methionine and valine do not affect the folding or stability of the native structure, implying that its influence takes place at downstream stages in the disease. Based on the broadening of the signals of Met-129 in solid-state NMR measurements of the Y145X stop mutant, it was suggested that Met-129 is located in a flexible loop (24). In contrast, using a combination of H/D exchange coupled to NMR spectroscopy and hydroxyl radical probing detected by mass spectrometry, we demonstrate that the amide proton of Met-129 has slow solvent exchange in the fibrillar state and that its side chain becomes buried upon aggregation into amyloid. We therefore attribute the line broadening observed in solid-state NMR experiments for residues 121-141 (25) to dynamics between different filaments. At the same time, a high rigidity of a single PrP filament is supported by the finding that submicrosecond time scale dynamics are uniform across the amyloid core (25).

In agreement with the burial of Met-129 in the amyloid core, the presence of a valine residue at position 129 strongly enhanced aggregation of humPrP(108-143) (Fig. 5A). The strong effect of the Val-129 substitution might at first sight appear surprising as the Val-129 variant of humPrP(23-144) converted to amyloid fibrils only slightly faster than Met-129 in previous in vitro studies (49). We attribute the differences to the entropic barrier provided by the unstructured residues $23-108$ thereby masking differences between different PrP variants. In support of an entropic barrier, aggregation of humPrP(23-144) and humPrP(23-159) requires several fold higher concentrations than humPrP(108-143) (Fig. 5A) (22). Importantly, both the Met-129 and the Val-129 humPrP(108-143) peptide displayed a shorter nucleation phase than 
their 1:1 combination modeling the heterozygous situation. Indeed, humans that are heterozygote at codon 129 of the prion protein gene are relatively protected from prion diseases, and homozygotes, either methionine or valine, are 2- 4-fold more susceptible (50).
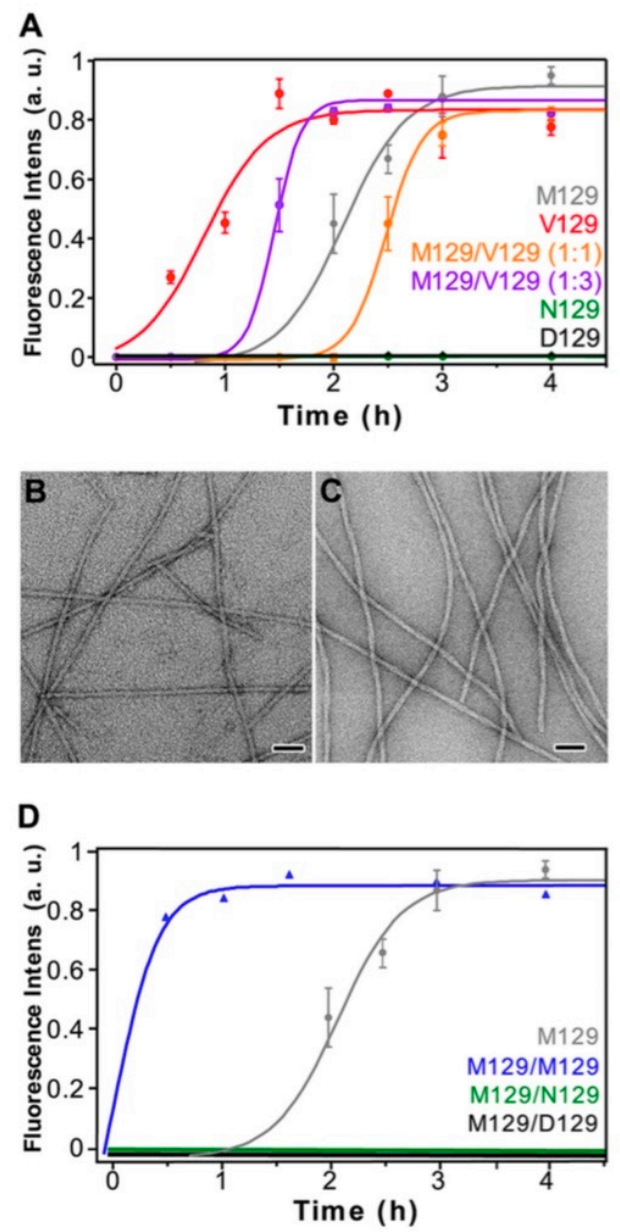

FIGURE 5. Importance of residue 129 for aggregation of humPrP(108-143). $A$, fibrillization kinetics of humPrP(108-143) variants. Error bars correspond to S.D. (at least three data sets). $B$, electron micrograph of wild-type humPrP(108-143). The average diameter was 7-9 $\mathrm{nm}$. $C$, electron micrograph of M109K humPrP(108-143). The black bar indicates $200 \mathrm{~nm}$. D, seeding fibrillization of Met-129, M129D, and M129N humPrP(108-143) by amyloid seeds of Met-129 humPrP(108-143). The concentration of the seed was 5\%. Data for Met-129 humPrP(108-143) without seed 
(gray) were taken from panel A.

\section{TABLE 1}

Quantification of aggregate formation of wild-type and M109K humPrP(108143) by centrifugation and protein quantification using UV absorbance.

\begin{tabular}{llllll}
\hline & \multicolumn{2}{c}{$2.7 \mathrm{~h}$} & & \multicolumn{2}{c}{$24 \mathrm{~h}$} \\
\cline { 2 - 3 } \cline { 5 - 6 } Peptide & \% in pellet & ThT intensity & & \% in pellet & Tht intensity \\
\hline WT & 58 & 437.5 & & 62 & 418 \\
M109K & 48 & 389 & 63 & 455.5 \\
\hline
\end{tabular}

The finding that the Val-129 variant aggregates faster than Met-129 of humPrP(23$144)$ is in contrast to previous aggregation experiments using recombinant $\operatorname{Pr} \mathrm{P}(90$ 231), where methionine is more aggregation-promoting than valine (51).

Besides differences in the experimental settings, an explanation may be that the Cterminally truncated mutants aggregate to a different prion type than the $\mathrm{N}$-terminally truncated one. From large epidemiological studies, we know that $87 \%$ of the methionine-homozygous sporadic Creutzfeldt-Jakob disease subjects accumulate prion type 1 and $97.5 \%$ of the valine homozygous Creutzfeldt-Jakob disease subjects accumulate prion type 2 (2). Taking this into account, the observed structure, starting at 109-112 and ending at 140-142, may represent the structural motif of prion type 2 .

In summary, we demonstrated that the polymorphic residue 129 is deeply buried in the amyloid core of stop mutants of the human prion protein. In line with its burial in the amyloid core structure, the identity of the 129 side chain is important for aggregation into amyloid fibrils. Our data support a critical role of the polymorphic residue 129 in the packing of protein chains into prion particles. 


\section{REFERENCES}

1. Aguzzi,A.,Sigurdson,C.,and Heikenwaelder,M.(2008)Molecular mechanisms of prion pathogenesis. Annu. Rev. Pathol. 3, 11-40

2. Parchi, P.,Giese,A.,Capellari,S.,Brown,P.,Schulz-Schaeffer,W.,Windl,O., Zerr, I., Budka, H., Kopp, N., Piccardo, P., Poser, S., Rojiani, A., Streichem- berger, N., Julien, J., Vital, C., Ghetti, B., Gambetti, P., and Kretzschmar, H. (1999) Classification of sporadic Creutzfeldt-Jakob disease based on molecular and phenotypic analysis of 300 subjects. Ann. Neurol 46, $224-233$

3. Wemheuer, W.M., Benestad, S.L.,Wrede,A., Schulze-Sturm,U., Wemheuer, W. E., Hahmann, U., Gawinecka, J., Schutz, E., Zerr, I., Brenig, B., Bratberg, B., Andreoletti, O., and Schulz-Schaeffer, W. J. (2009) Similarities between forms of sheep scrapie and Creutzfeldt-Jakob disease are encoded by distinct prion types. Am. J. Pathol. 175, 2566-2573

4. Parchi, P., Zou, W., Wang, W., Brown, P., Capellari, S., Ghetti,B., Kopp, N., Schulz-Schaeffer, W. J., Kretzschmar, H. A., Head, M. W., Ironside, J. W., Gambetti, P., and Chen, S. G. (2000) Genetic influence on the structural variations of the abnormal prion protein. Proc. Natl. Acad. Sci. U.S.A. 97, $10168-10172$

5. Schulz-Schaeffer, W. J., Giese, A., Windl, O., and Kretzschmar, H. A. (1996) Polymorphism at codon 129 of the prion protein gene determines cerebellar pathology in Creutzfeldt-Jakob disease. Clin. Neuropathol 15, 353-357

6. Heinemann, U., Krasnianski, A., Meissner, B., Varges,D., Kallenberg, K., SchulzSchaeffer, W. J., Steinhoff, B. J., Grasbon-Frodl, E. M., Kretzschmar, H. A., and Zerr, I. (2007) Creutzfeldt-Jakob disease in Germany: a prospective 12-year surveillance. Brain 130, $1350-1359$

7. Telling, G. C. (2011) Transgenic mouse models and prion strains. Top Curr. Chem. 305, $79-99$

8. Wuthrich,K., and Riek, R.(2001)Three-dimensional structures of prion proteins. Adv. Protein Chem. 57, 55- 82

9. Wille, H., Bian, W., McDonald, M., Kendall, A., Colby, D. W., Bloch, L., Ollesch, J., Borovinskiy, A. L., Cohen, F. E., Prusiner, S. B., and Stubbs, G. (2009) Natural and synthetic prion structure from x-ray fiber diffraction. Proc. Natl. Acad. Sci. U.S.A. 106, $16990-16995$

10. Paramithiotis, E., Pinard,M., Lawton,T., LaBoissiere,S., Leathers,V.L., Zou, W. Q., Estey, L. A., Lamontagne, J., Lehto, M. T., Kondejewski, L. H., Francoeur, G. P., Papadopoulos, M., Haghighat, A., Spatz, S. J., Head, M., Will, R., Ironside, J., O’Rourke, K., Tonelli, Q., Ledebur, H. C., Chakrabartty, A., and Cashman, N. R. (2003) A prion protein epitope selective for the pathologically misfolded conformation. Nat. Med. 9, 893-899

11. Smirnovas,V., Baron,G.S., Offerdahl,D.K., Raymond,G.J., Caughey,B., and Surewicz, W. K. (2011) Structural organization of brain-derived mammalian prions 
examined by hydrogen-deuterium exchange. Nat. Struct. Mol. Biol. 18, $504-506$

12. Govaerts, C., Wille, H., Prusiner, S. B., and Cohen, F. E. (2004) Evidence for assembly of prions with left-handed beta-helices into trimers. Proc. Natl. Acad. Sci. U.S.A. 101, 8342- 8347

13. Wille, H., Govaerts, C., Borovinskiy, A., Latawiec, D., Downing, K. H., Cohen, F. E., and Prusiner, S. B. (2007) Electron crystallography of the scrapie prion protein complexed with heavy metals. Arch Biochem. Bio- phys. 467, $239-248$

14. Kunes, K. C., Clark, S. C., Cox, D. L., and Singh, R. R. (2008) Left handed helix models for mammalian prion fibrils. Prion 2, 81-90

15. Lu, X., Wintrode, P. L., and Surewicz, W. K. (2007) Beta-sheet core of human prion protein amyloid fibrils as determined by hydrogen/deuterium exchange. Proc. Natl. Acad. Sci. U.S.A. 104, $1510-1515$

16. Nazabal, A., Hornemann, S., Aguzzi, A., and Zenobi, R. (2009) Hydrogen/deuterium exchange mass spectrometry identifies two highly protected regions in recombinant full-length prion protein amyloid fibrils. J. Mass Spectrom 44, 965-977

17. Damo, S. M., Phillips, A. H., Young, A. L., Li, S., Woods, V. L., Jr., and Wemmer, D. E. (2010) Probing the conformation of a prion protein fibril with hydrogen exchange. J. Biol. Chem. 285, 32303-32311

18. DeMarco, M. L., and Daggett, V. (2004) From conversion to aggregation: protofibril formation of the prion protein. Proc. Natl. Acad. Sci. U.S.A. 101, 2293 2298

19. Tateishi, J., Kitamoto, T., Kretzschmar, H., and Mehraein, P. (1996) Immunohistological evaluation of Creutzfeldt-Jakob disease with reference to the type PrPres deposition. Clin. Neuropathol 15, 358-360

20. Kundu, B., Maiti, N. R., Jones, E. M., Surewicz, K. A., Vanik, D. L., and Surewicz, W. K. (2003) Nucleation-dependent conformational conversion of the Y145Stop variant of human prion protein: structural clues for prion propagation. Proc. Natl. Acad. Sci. U.S.A. 100, $12069-12074$

21. Vanik, D. L., Surewicz, K. A., and Surewicz, W. K. (2004) Molecular basis of barriers for interspecies transmissibility of mammalian prions. Mol. Cell 14, 139-145

22. Watzlawik, J., Skora, L., Frense, D., Griesinger, C., Zweckstetter, M., SchulzSchaeffer, W. J., and Kramer, M. L. (2006) Prion protein helix 1 promotes aggregation but is not converted into beta-sheet. J. Biol. Chem. 281, 30242-30250

23. Jayadev, S., Nochlin, D., Poorkaj, P., Steinbart, E. J., Mastrianni, J. A., Montine, T. J., Ghetti, B., Schellenberg, G. D., Bird, T. D., and Leverenz, J. B. (2011) Familial prion disease with Alzheimer disease-like Tau pathology and clinical phenotype. Ann. Neurol 69, 712-720

24. Helmus, J. J., Surewicz, K., Nadaud, P. S., Surewicz, W. K., and Jaroniec, C. P. 
(2008) Molecular conformation and dynamics of the Y145Stop variant of human prion protein in amyloid fibrils. Proc. Natl. Acad. Sci. U.S.A. 105, $6284-6289$

25. Helmus, J. J., Surewicz, K., Surewicz, W. K., and Jaroniec, C. P. (2010) Conformational flexibility of Y145Stop human prion protein amyloid fibrils probed by solid-state nuclear magnetic resonance spectroscopy. J. Am. Chem. Soc. 132, 2393-2403

26. Helmus, J. J., Surewicz, K., Apostol, M. I., Surewicz, W. K., and Jaroniec, C. P. (2011) Intermolecular alignment in Y145Stop human prion protein amyloid fibrils probed by solid-state NMR spectroscopy. J. Am. Chem. Soc. 133, $13934-13937$

27. Liemann, S., and Glockshuber, R. (1999) Influence of amino acid substitutions related to inherited human prion diseases on the thermodynamic stability of the cellular prion protein. Biochemistry 38, $3258-3267$

28. Cho, M. K., Kim, H. Y., Fernandez, C. O., Becker, S., and Zweckstetter, M. (2011) Conserved core of amyloid fibrils of wild type and A30P mutant alphasynuclein. Protein Sci. 20, 387-395

29. Delaglio, F., Grzesiek, S., Vuister, G. W., Zhu, G., Pfeifer, J., and Bax, A. (1995) NMRPipe: a multidimensional spectral processing system based on UNIX pipes. $J$. Biomol. NMR 6, 277-293

30. Bax, A., and Grzesiek, S. (1993) Methodological advances in protein. Acc. Chem. Res. 26, 131-138

31. Panchal, S. C., Bhavesh, N. S., and Hosur, R. V. (2001) Improved 3D triple resonance experiments, $\mathrm{HNN}$ and $\mathrm{HN}(\mathrm{C}) \mathrm{N}$, for $\mathrm{HN}$ and ${ }^{15} \mathrm{~N}$ sequential correlations in $\left({ }^{13} \mathrm{C},{ }^{15} \mathrm{~N}\right)$ labeled proteins: application to unfolded proteins. J. Biomol. NMR 20, 135147

32. Stejskal, E. O., and Tanner, J. E. (1965) Spin diffusion measurements: spin echoes in the presence of a time-dependent field gradient. J. Chem. Phys. 42, $288-292$

33. Wu, D. H., Chen, A. D., and Johnson, C. S. (1995) An improved diffusion-ordered spectroscopy experiment incorporating bipolar-gradient pulses. J. Magn. Reson. A $115,260-264$

34. Sharp, J. S., Becker, J. M., and Hettich, R. L. (2003) Protein surface mapping by chemical oxidation: structural analysis by mass spectrometry. Anal. Biochem. 313, $216-225$

35. Rappsilber, J., Ishihama, Y., and Mann, M. (2003) Stop and go extraction tips for matrix-assisted laser desorption/ionization, nanoelectrospray, and LC/MS sample pretreatment in proteomics. Anal. Chem. 75, 663- 670

36. Cox, J., and Mann, M. (2008) MaxQuant enables high peptide identification rates, individualized p.p.b.-range mass accuracies, and proteome- wide protein quantification. Nat. Biotechnol. 26, 1367-1372

37. Cox, J., Neuhauser, N., Michalski, A., Scheltema, R. A., Olsen, J. V., and Mann, M. (2011) Andromeda: a peptide search engine integrated into the MaxQuant 
environment. J. Proteome Res. 10, $1794-1805$

38. Charvatova, O., Foley, B. L., Bern, M. W., Sharp, J. S., Orlando, R., and Woods, R. J. (2008) Quantifying protein interface footprinting by hydroxyl radical oxidation and molecular dynamics simulation: application to galectin-1. J. Am. Soc. Mass Spectrom 19, 1692-1705

39. Perrin, F. (1934) Brownian motion of an ellipsoid. I. Dielectric dispersion for ellipsoidal molecules. J. Phys. Radium 5, 497-511

40. Hoshino, M., Katou, H., Hagihara, Y., Hasegawa, K., Naiki, H., and Goto, Y. (2002) Mapping the core of the beta-microglobulin amyloid fibril by H/D exchange. Nat. Struct. Biol. 9, 332-336

41. Xu, G., and Chance, M. R. (2007) Hydroxyl radical-mediated modification of proteins as probes for structural proteomics. Chem. Rev. 107, 3514-3543

42. Breydo, L., Bocharova, O. V., Makarava, N., Salnikov, V. V., Anderson, M., and Baskakov, I. V. (2005) Methionine oxidation interferes with conversion of the prion protein into the fibrillar proteinase K-resistant conformation. Biochemistry 44, 15534 $-15543$

43. Ghetti, B., Piccardo, P., Spillantini, M. G., Ichimiya, Y., Porro, M., Perini, F., Kitamoto, T., Tateishi, J., Seiler, C., Frangione, B., Bugiani, O., Giaccone, G., Prelli, F., Goedert, M., Dlouhy, S. R., and Tagliavini, F. (1996) Vascular variant of prion protein cerebral amyloidosis with Tau-positive neurofibrillary tangles: the phenotype of the stop codon 145 mutation in PRNP. Proc. Natl. Acad. Sci. U.S.A. 93, $744-748$

44. Jansen, C., Parchi, P., Capellari, S., Vermeij, A. J., Corrado, P., Baas, F., Strammiello, R., van Gool, W. A., van Swieten, J. C., and Rozemuller, A. J. (2010) Prion protein amyloidosis with divergent phenotype associated with two novel nonsense mutations in PRNP. Acta Neuropathol 119, $189-197$

45. Tagliavini, F., Prelli, F., Ghiso, J., Bugiani, O., Serban, D., Prusiner, S. B., Farlow, M. R., Ghetti, B., and Frangione, B. (1991) Amyloid protein of GerstmannStraussler-Scheinker disease (Indiana kindred) is an $11 \mathrm{kD}$ fragment of prion protein with an N-terminal glycine at codon 58. EMBO J. 10, 513-519

46. Wopfner, F., Weidenhofer, G., Schneider, R., von Brunn, A., Gilch, S., Schwarz, T. F., Werner, T., and Schatzl, H. M. (1999) Analysis of 27 mammalian and 9 avian PrPs reveals high conservation of flexible regions of the prion protein. J. Mol. Biol. 289, $1163-1178$

47. Holscher, C., Delius, H., and Burkle, A. (1998) Overexpression of non convertible $\operatorname{PrP}^{\mathrm{c}} 114-121$ in scrapie-infected mouse neuroblastoma cells leads to trans-dominant inhibition of wild-type $\mathrm{PrPSc}^{\mathrm{Sc}}$ accumulation. J. Virol. 72, 1153-1159

48. Skora, L., and Zweckstetter, M. (2012) Determination of amyloid core structure using chemical shifts. Protein Sci. 21, $1948-1953$

49. Jones, E. M., Surewicz, K., and Surewicz, W. K. (2006) Role of N-terminal familial mutations in prion protein fibrillization and prion amyloid propagation in 
vitro. J. Biol. Chem. 281, $8190-8196$

50. Palmer, M. S., Dryden, A. J., Hughes, J. T., and Collinge, J. (1991) Homozygous prion protein genotype predisposes to sporadic Creutzfeldt-Jakob disease. Nature 352, $340-342$

51. Tahiri-Alaoui, A., Gill, A. C., Disterer, P., and James, W. (2004) Methionine 129 variant of human prion protein oligomerizes more rapidly than the valine 129 variant: implications for disease susceptibility to Creutzfeldt-Jakob disease. J. Biol. Chem. 279, $31390-31397$ 


\section{SUPPORTING INFORMATION}

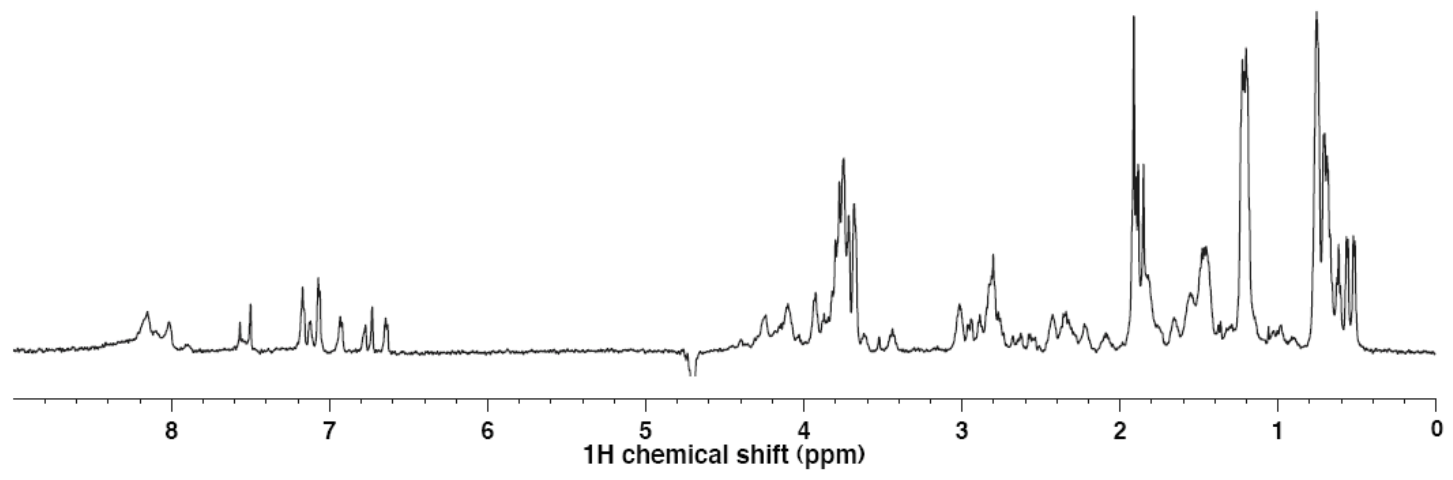

Figure S1. One-dimensional proton NMR spectrum of humPrP(108-143). The small dispersion in the amide proton $(\sim 8.2 \mathrm{ppm})$ and methyl region $(0-1 \mathrm{ppm})$ demonstrates that monomeric humPrP(108-143) is disordered in solution. 
a)

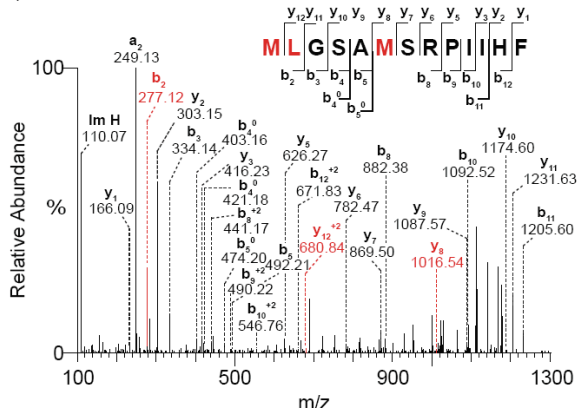

c)

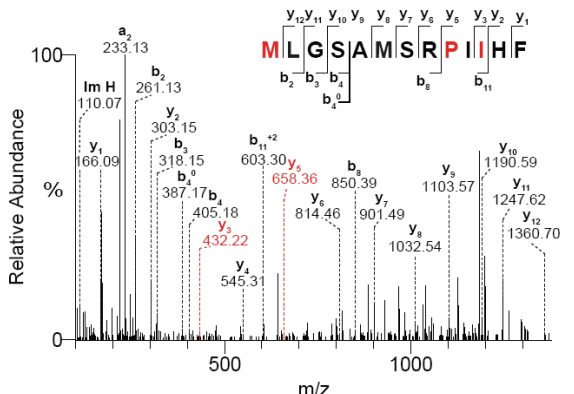

e)
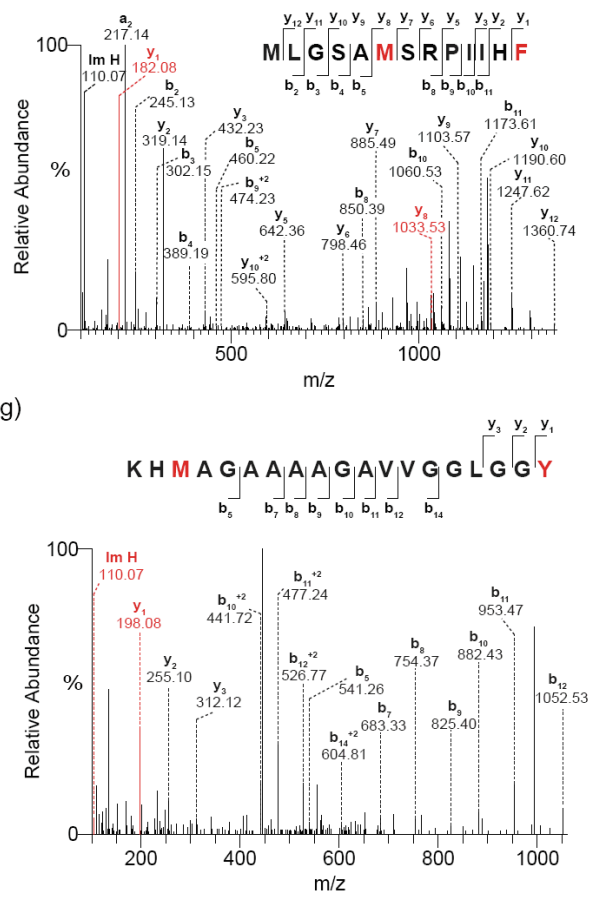

b)

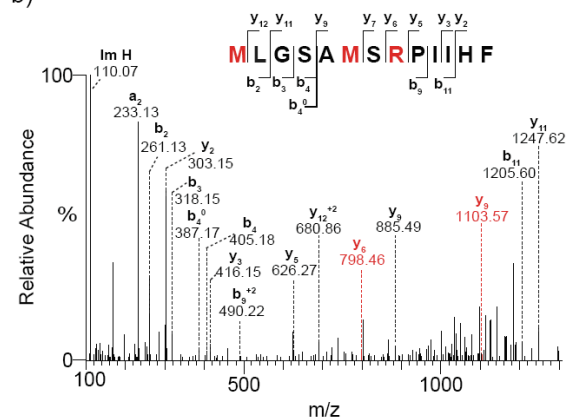

d)
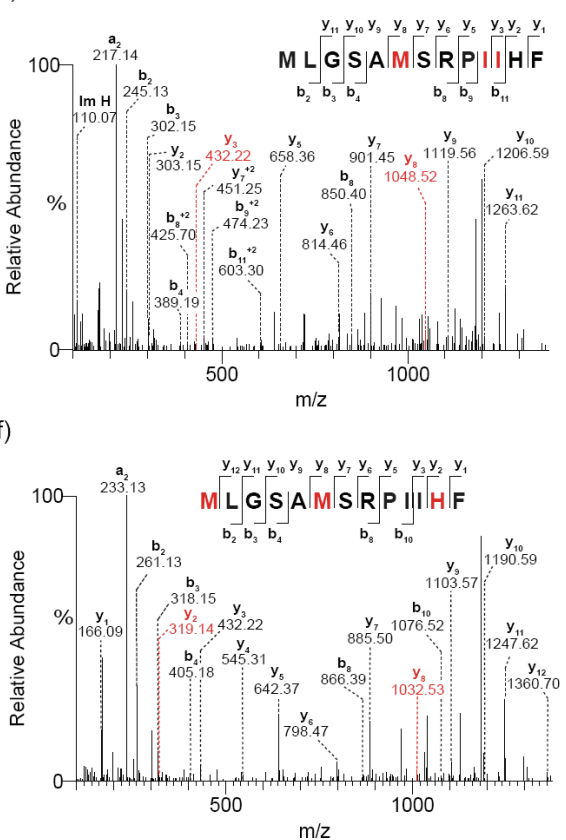

h)

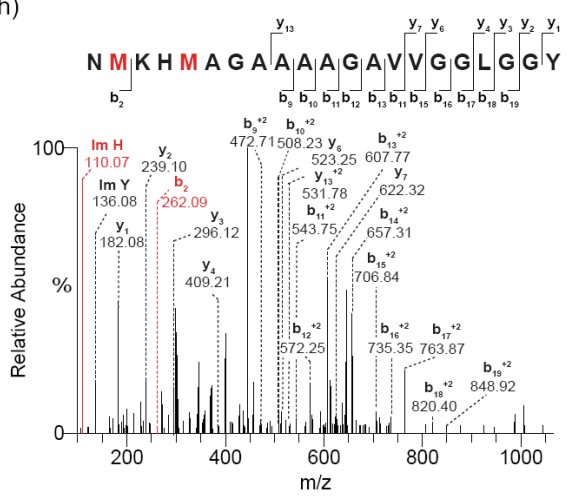

Figure S2. Tandem MS spectra of modified (oxidized) humPrP(108-143) peptides. The oxidized amino acid and its corresponding fragment ions containing the modification are depicted in red, $\mathrm{b}$ and $\mathrm{y}$ ions indicate fragmentation of the peptide bond conserving the charge to the $\mathrm{N}$ - or $\mathrm{C}$ - terminus, respectively; subindex 0 indicates loss of water.(i.e b-H2O) a-f) Oxidized amino acids found in peptide 
129MLGSAMSRPIIHF141: a) M129, L130, M134 b) M129, M134 and R136; c) M129, P137 and I139; d) M134, I138, I139; e) M134 and F141 and f) M129, M134 and H140. g-h) Oxidized amino acids in the peptide 109-128: g) M112 and Y128 are oxidized, note the immonium ion of $\mathrm{H}$ (non modified); h) Modification in M109 and M112; of note immonium ions of H111 and Y128 are non-oxidized indicating that M112 is the actual oxidized residue. Spectra a-f) are from fibrillar samples, whereas spectra g-h) correspond to monomeric samples. 


\section{Luis Eduardo Fonseca-Ornelas}

Jüdenstraße 41. 37073. Göttingen, Germany.

lufo@nmr.mpibpc.mpg.de

Education

$$
\begin{aligned}
\text { Oct } 2012-D e c & \text { Georg-August-Universität Göttingen } \\
2015 & \begin{array}{l}
\text { Doctor of Philosophy, Structural Biology } \\
\text { Göttingen, Germany }
\end{array}
\end{aligned}
$$

Aug 2008 - Jul 2011 Universidad Nacional Autónoma de México

Master of Science, Biochemistry

Cuernavaca, Mexico

Aug 2004 - Jul 2008 Universidad Nacional Autónoma de México

Bachelor of Science, Biology

Mexico City, Mexico

Research Experience

Nov 2011 - present

\section{PhD Student}

Max Planck Institute for Biophysical Chemistry, Department of NMRbased Structural Biology

Göttingen, Germany

Aug 2008-Aug 2011 Master's Student

Universidad Nacional Autónoma de México, Departament of Developmental Genetics and Molecular Physiology

Cuernavaca, Morelos, Mexico

Aug 2007 - Jul 2008 Bachelors Student.

Universidad Nacional Autónoma de México, Departament of Developmental Genetics and Molecular Physiology

Cuernavaca, Morelos, Mexico

Aug 2006 - Jul 2007 Rotation student

Universidad Nacional Autónoma de México, Department of Neurodevelopment and Physiology

Mexico City, Mexico

May 2004 -Jul 2004 Summer Student

Neurobiology Institute, Neuromorphotoxicology

Santiago de Querétaro, Querétaro, Mexico

Awards \& Grants

Oct 2011 Scholarship: Marie Curie fellowship 
Sep 2008 Scholarship: Mexican Council for Science \& Technology. Scholarship for Masters studies.

Apr 2008 Award: Highest score in entrance examination for Masters in Biochemical Sciences.

Aug 2007 Scholarship: Scholarship for outstanding students.

Aug 2004 Award: Youth towards research excellence program. Best-written report. Best poster.

Skills \& Activities

Skills Parkinson's Disease, Amyloid proteins, Neurodegenerative Diseases, alpha-Synuclein, Drosophila, Toxicity, Neurobiology, Prion Protein, Protein Aggregation, Prion Diseases

Languages English (proficient), Spanish (native), German (conversational)

\section{Publications}

Luis Fonseca-Ornelas, Sybille E Eisbach, Maria Paulat, Karin Giller, Claudio O Fernández, Tiago F Outeiro, Stefan Becker, Markus Zweckstetter: Small molecule-mediated stabilization of vesicle-associated helical $\alpha$-synuclein inhibits pathogenic misfolding and aggregation. Nature Communications 12/2014; 5:5857. DOI:10.1038/ncomms6857

Lukasz Skora, Luis Fonseca-Ornelas, Romina V Hofele, Dietmar Riedel, Karin Giller, Jens Watzlawik, Walter J Schulz-Schaeffer, Henning Urlaub, Stefan Becker, Markus Zweckstetter: Burial of the Polymorphic Residue 129 in Amyloid Fibrils of Prion Stop Mutants. Journal of Biological Chemistry 12/2013; 288(5). DOI:10.1074/jbc.M112.423715

René Hernández-Vargas, Luis Fonseca-Ornelas, Ignacio López-González, Juan Riesgo-Escovar, Mario Zurita, Enrique Reynaud: Synphilin suppresses $\alpha$ synuclein neurotoxicity in a Parkinson's disease Drosophila model. Genesis 05/2011; 49(5):392-402. DOI:10.1002/dvg.20740 